J. Anal. Math. 92 (2004), 1-79.

\title{
Séries trigonométriques à coefficients arithmétiques
}

\author{
R. de la Bretèche \& G. Tenenbaum
}

\section{Sommaire}

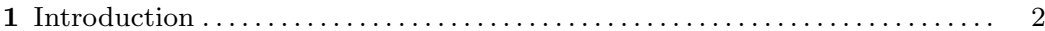

2 Estimations fondamentales : $\nabla(\vartheta ; y)$ et $B(\vartheta ; y) \ldots \ldots \ldots \ldots \ldots \ldots \ldots$

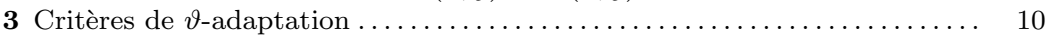

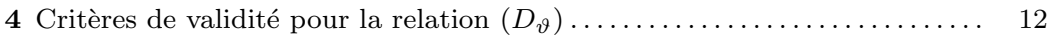

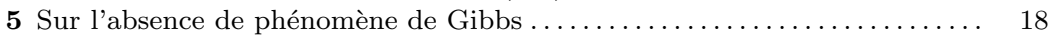

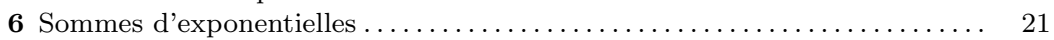

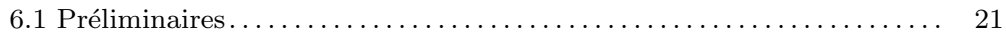

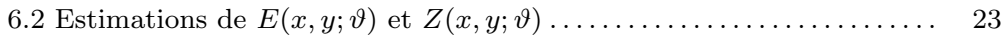

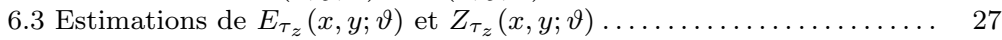

$7 P$-convergence de $V(\mu ; \vartheta)$ : preuve du Théorème $2.1 \ldots \ldots \ldots \ldots \ldots \ldots \ldots . \ldots \ldots$

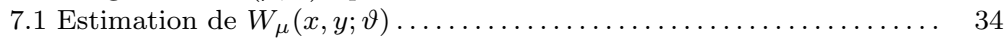

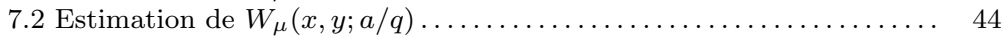

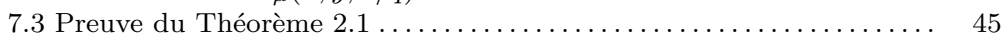

$8 P$-convergence de $U(\mathbf{1} ; \vartheta)$ : preuve du Théorème $2.2 \ldots \ldots \ldots \ldots \ldots \ldots \ldots . \ldots 46$

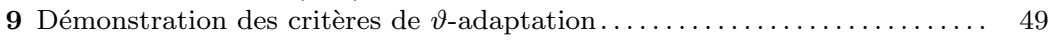

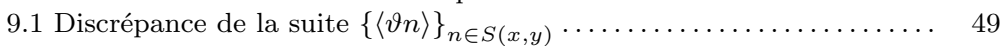

9.2 Critère de $\vartheta$-adaptation lorsque $f \in \mathcal{L}^{\alpha}\left(\mathbb{N}^{*}\right)$ : preuve du Théorème 3.152

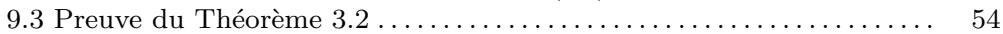

9.4 Critère de $\vartheta$-adaptation lorsque $\vartheta \in \mathbb{Q}:$ preuve du Théorème $3.4 \ldots 56$ 9.5 Critère de $\vartheta$-adaptation lorsque $g \in \mathcal{L}^{\alpha}\left(\mathbb{N}^{*}\right)$ : preuve du Théorème 3.556

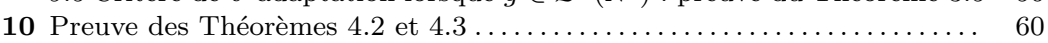

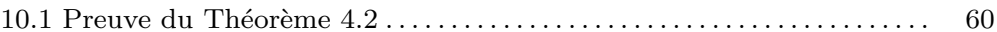

$10.2\left(D_{\vartheta}\right)$ pour $(f, g)=(\log , \Lambda):$ preuve du Théorème $4.3 \ldots \ldots \ldots \ldots \ldots 61$

11 Le cas $(f, g)=(\tau, \mathbf{1}):$ preuve du Théorème $4.4 \ldots \ldots \ldots \ldots \ldots \ldots \ldots 64$

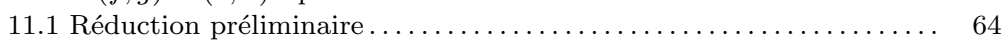

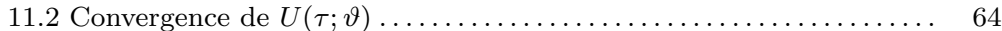

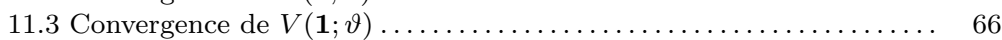

$12\left(D_{\vartheta}\right)$ pour $(f, g)=(\Lambda,-\mu \log ):$ preuve du Théorème $4.5 \ldots \ldots \ldots \ldots \ldots 68$

13 Cas des fonctions multiplicatives $\ldots \ldots \ldots \ldots \ldots \ldots \ldots \ldots \ldots \ldots \ldots \ldots$

13.1 Preuve du Théorème 4.6: $(f, g) \in \mathcal{F}_{A} \times \mathcal{F}_{A}$ et $\vartheta \in \mathbb{R} \backslash \mathbb{Q} \ldots \ldots \ldots .69$

13.2 Preuve du Théorème 4.7: $g \in \mathcal{F}_{A}^{*}$ et $\vartheta \in \mathbb{Q} \ldots \ldots \ldots \ldots \ldots \ldots \ldots \ldots$

13.3 Preuve du Théorème $4.8:(f, g)=\left(\tau_{z+1}, \tau_{z}\right) \ldots \ldots \ldots \ldots \ldots \ldots \ldots$ 


\section{Introduction}

Soit $\mathbb{T}:=\mathbb{R} / \mathbb{Z}$. Considérons une fonction 1-périodique $F \in L^{1}(\mathbb{T})$, d'intégrale nulle et partout somme de sa série de Fourier, soit

$$
F(\vartheta)=\sum_{n=1}^{\infty}\left\{a_{F}(n) \cos (2 \pi n \vartheta)+b_{F}(n) \sin (2 \pi n \vartheta)\right\} \quad(\vartheta \in \mathbb{R}) .
$$

On a alors formellement, pour toute fonction arithmétique $h$,

$$
\sum_{n=1}^{\infty} h(n) F(n \vartheta)=\sum_{n=1}^{\infty}\left\{\left(a_{F} * h\right)(n) \cos (2 \pi n \vartheta)+\left(b_{F} * h\right)(n) \sin (2 \pi n \vartheta)\right\}
$$

où les convolutions sont prises au sens de Dirichlet. Le problème de décider dans quels cas cette égalité formelle prend un sens analytique a été abordé par Davenport dans [7] et [8]. Il considère essentiellement le cas où $F=B$, la somme de la série de Fourier de la première fonction de Bernoulli, $B_{1}(\vartheta):=\langle\vartheta\rangle-\frac{1}{2}$, où $\langle\vartheta\rangle$ désigne la partie fractionnaire du nombre réel $\vartheta$ - on a donc

$$
B(\vartheta)= \begin{cases}\langle\vartheta\rangle-\frac{1}{2} & \text { si } \vartheta \notin \mathbb{Z} \\ 0 & \text { si } \vartheta \in \mathbb{Z}\end{cases}
$$

Ce choix s'explique d'une part par l'intérêt arithmétique de la fonction $B_{1}$, immédiatement liée à la fonction partie entière, et d'autre part par le fait bien connu que la convergence de la série des coefficients est meilleure dans le cas des séries de sinus que dans celui des séries de cosinus, ${ }^{(1)}$ ce qui augmente a priori la probabilité de convergence de $(1 \cdot 1)$. On a

$$
B(\vartheta)=-\sum_{m=1}^{\infty} \frac{\sin (2 \pi m \vartheta)}{\pi m}
$$

Normalisons la fonction $h$ de $(1 \cdot 1)$ en posant $h(n)=g(n) / n$ pour $n \geqslant 1$. Nous pouvons alors réécrire $(1 \cdot 1)$ sous la forme

$$
\sum_{m=1}^{\infty} \frac{f(m)}{\pi m} \sin (2 \pi m \vartheta)+\sum_{n=1}^{\infty} \frac{g(n)}{n} B(n \vartheta)=0
$$

où les fonctions arithmétiques $f$ et $g$ sont liées par la relation de convolution

$$
f=g * \mathbf{1}, \quad \text { i.e. } \quad f(n)=\sum_{d \mid n} g(d) \quad(n \geqslant 1) .
$$

1. Si $\sum_{n \geqslant 1} a_{n} \sin (2 \pi n \vartheta)$ est une série de Fourier à coefficients de signe constant, on a nécessairement $\sum_{n \geqslant 1}\left|a_{n}\right| / n<\infty$ : voir par exemple [18], Corollary I.4.2. 
Dans tout cet article, nous écrivons

$$
(f, g) \in D_{\vartheta}
$$

pour signifier que la relation $\left(D_{\vartheta}\right)$ est satisfaite pour le couple de fonctions arithmétiques $(f, g)$ vérifiant $(1 \cdot 3)$. En outre, par commodité de référence, nous désignons respectivement par $U(f ; \vartheta)$ et $V(g ; \vartheta)$ les séries en $f$ et $g$ de $\left(D_{\vartheta}\right)$, soit formellement

$$
U(f ; \vartheta):=\sum_{m \geqslant 1} \frac{f(m)}{\pi m} \sin (2 \pi m \vartheta), \quad V(g ; \vartheta):=\sum_{n \geqslant 1} \frac{g(n)}{n} B(n \vartheta) .
$$

Nous remarquons d'emblée que, si $(f, g) \in D_{\vartheta}$ pour tout $\vartheta$ et si la convergence des deux membres est uniformément bornée en $\vartheta$, alors $(f * h, g * h) \in D_{\vartheta}$ pour tout $\vartheta$ dès que

$$
\sum_{n \geqslant 1} \frac{|h(n)|}{n}<\infty
$$

Cela découle immédiatement du théorème de Lebesgue, et nous omettons les détails, qui sont standard.

Une autre application immédiate du théorème de Lebesgue dans ce contexte consiste à remarquer que, si

$$
F(\vartheta)=\sum_{n \geqslant 1}\left\{a_{F}(n) \cos (2 \pi n \vartheta)+b_{F}(n) \sin (2 \pi n \vartheta)\right\}
$$

est une fonction à variation bornée sur $\mathbb{T}$ qui est en tout point somme de sa série de Fourier, ${ }^{(2)}$ et si $(f, g)$ est un couple de fonctions arithmétiques satisfaisant $\left(D_{\vartheta}\right)$ pour tout $\vartheta \in \mathbb{R}$ avec une convergence uniformément bornée des séries $U(f ; \vartheta)$ et $V(g ; \vartheta)$, alors on a

$$
\sum_{m \geqslant 1} f(m)\left\{a_{F}(m) \cos (2 \pi m \vartheta)+b_{F}(m) \sin (2 \pi m \vartheta)\right\}+\sum_{n \geqslant 1} g(n) F_{n}(\vartheta)=0
$$

où

$$
F_{n}(\vartheta):=\frac{1}{n} \sum_{1 \leqslant j \leqslant n} F\left(\vartheta+\frac{j}{n}\right)=\sum_{\substack{k \geqslant 1 \\ k \equiv 0(\bmod n)}}\left\{a_{F}(k) \cos (2 \pi k \vartheta)+b_{F}(k) \sin (2 \pi k \vartheta)\right\}
$$

2. D'après un théorème de Jordan, il suffit pour cela qu'elle soit normalisée par $F(\vartheta)=\frac{1}{2} F(\vartheta+)+\frac{1}{2} F(\vartheta-)$. 
est la somme de Riemann associée à $F$. Cela résulte immédiatement de la représentation

$$
F_{n}(\vartheta)=\frac{1}{n} \int_{\mathbb{T}} B(n \vartheta-n v) \mathrm{d} F(v) \quad(n \geqslant 1),
$$

et nous omettons les détails.

Le développement de Fourier classique $(1 \cdot 2)$ coïncide avec $\left(D_{\vartheta}\right)$ lorsque

$$
(f, g)=(\mathbf{1}, \boldsymbol{\delta}),
$$

où $\delta$ désigne l'élément neutre de la convolution. ${ }^{(3)}$ Dans [7], [8], Davenport donne un certain nombre de cas de validité de $\left(D_{\vartheta}\right)$ et prouve en particulier que $(\boldsymbol{\delta}, \mu) \in D_{\vartheta}$ pour tout nombre réel $\vartheta$. Plus précisément, en établissant entre autres, pour chaque $A>0$ fixé, la majoration

$$
\sup _{\vartheta \in \mathbb{R}}\left|\sum_{n \leqslant y} \mu(n) \mathrm{e}^{2 \pi i n \vartheta}\right| \ll \frac{y}{(\log y)^{A}} \quad(y \geqslant 2),
$$

il obtient la forme quantitative

$$
\Delta(\vartheta ; y):=\frac{\sin (2 \pi \vartheta)}{\pi}+\sum_{n \leqslant y} \frac{\mu(n)}{n} B(n \vartheta) \ll \frac{1}{(\log y)^{A}}
$$

valable, pour tout $A>0$, uniformément en $\vartheta \in \mathbb{R} .^{(4)}$

Posons plus généralement

$$
\Delta_{f}(\vartheta ; y):=\sum_{m \leqslant y} \frac{f(m)}{\pi m} \sin (2 \pi m \vartheta)+\sum_{n \leqslant y} \frac{g(n)}{n} B(n \vartheta),
$$

de sorte que $\Delta(\vartheta ; y)=\Delta_{\delta}(\vartheta ; y)$. En remplaçant, dans cette expression, $\sin (2 \pi m \vartheta) / \pi$ par

$$
\Delta(m \vartheta ; y / m)-\sum_{d \leqslant y / m} \frac{\mu(d)}{d} B(d m \vartheta)
$$

3. Défini par $\boldsymbol{\delta}(1)=1$ et $\boldsymbol{\delta}(n)=0$ si $n \geqslant 2$.

4. L'estimation conditionnelle de $\Delta(\vartheta ; y)$ pose également un problème intéressant encore partiellement ouvert. Sous l'hypothèse de Riemann généralisée, Baker et Harman [1] ont montré que le membre de gauche de $(1.6)$ est $<y^{(3 / 4)+o(1)}$. On peut en déduire facilement, en utilisant le résultat de Davenport, que $\sup _{\vartheta \in \mathbb{R}}|\Delta(\vartheta ; y)|<y^{-1 / 4+o(1)}$. En fait, pour toute fonction $\varepsilon_{y}$ positive et décroissante vérifiant $\int_{1}^{\infty} \varepsilon_{t} \mathrm{~d} t / t<\infty$ et telle que le membre

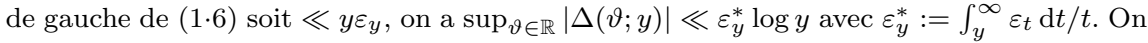
peut établir cela en utilisant la représentation $(7 \cdot 39)$, la majoration $(7 \cdot 41)$ et le fait que $\Delta(\vartheta, y)$ est dérivable en $\vartheta$, et de dérivée $\ll y \varepsilon_{y}$, sur tout intervalle ne contenant pas de point de la suite de Farey d'ordre $y$. 
pour chaque entier $m$ de $[1, y]$, nous obtenons l'identité

$$
\Delta_{f}(\vartheta ; y)=\sum_{m \leqslant y} \frac{f(m)}{m} \Delta(m \vartheta ; y / m)
$$

qui peut être utilisée, compte tenu de (1·7), pour donner des conditions suffisantes de validité de $\left(D_{\vartheta}\right)$. Il est clair, en effet, que l'on a $(f, g) \in D_{\vartheta}$ si, et seulement si, l'une des séries $U(f ; \vartheta), V(g ; \vartheta)$, converge et $\Delta_{f}(\vartheta ; y) \rightarrow 0$ lorsque $y \rightarrow \infty$. L'énoncé suivant, qui ne fait intervenir qu'une condition de croissance sur $f$, résulte immédiatement de (1.7) et (1.9).

Théorème 1.1. Soient $\vartheta \in \mathbb{R}$, et $(f, g)$ un couple de fonctions arithmétiques vérifiant (1-3). Alors, on a $(f, g) \in D_{\vartheta}$ dès que l'une au moins des deux conditions suivantes est réalisée :

(i) Les deux séries de $\left(D_{\vartheta}\right)$ sont convergentes et l'on a, pour un nombre réel $A>0$ convenable,

$$
\liminf _{y \rightarrow \infty} \sum_{m \leqslant y} \frac{|f(m)|}{m(\log 2 y / m)^{A}}=0 .
$$

(ii) L'une des deux séries de $\left(D_{\vartheta}\right)$ est convergente et l'on a

$$
\sum_{m \leqslant y}|f(m)|=o(y) \quad(y \rightarrow \infty) .
$$

Davenport montre dans [7] que l'on a $(\mathbf{1} * \lambda, \lambda) \in D_{\vartheta}$ pour tout $\vartheta \in \mathbb{R}$, où $\lambda$ désigne la fonction de Liouville; sa démonstration s'adapte plus généralement au cas $\sum_{n=1}^{\infty}|f(n)| / n<\infty$, qui est inclus dans l'assertion (i) de l'énoncé précédent.

Dans ce travail, nous nous proposons d'aborder le problème de la validité de $\left(D_{\vartheta}\right)$ en utilisant la notion nouvelle de $P$-convergence. Ce procédé de sommation a été formellement défini par Fouvry et Tenenbaum dans [14] ; la notion est comparable à celle de «densité multiplicative», introduite par Davenport et Erdős [11], qui recouvre essentiellement la même idée.

Rappelons les définitions essentielles dans un énoncé formel. Ici et dans tout l'article, nous désignons par $P(n)$ le plus grand facteur premier d'un entier générique $n$ avec la convention $P(1)=1$.

Définition $1.2([\mathbf{1 4}])$. On dit qu'une série de nombres complexes $\sum_{n=1}^{\infty} \alpha_{n}, P$ converge vers $\alpha$ si la série $\sum_{P(n) \leqslant y} \alpha_{n}$ converge pour tout $y \geqslant 2$, et si l'on a

$$
\sum_{P(n) \leqslant y} \alpha_{n}=\alpha+o(1) \quad(y \rightarrow \infty) .
$$


On dit qu'une série convergente $\sum_{n=1}^{\infty} \alpha_{n}$ est $P$-régulière si elle est $P$-convergente et si sa $P$-somme est égale à sa somme ordinaire, autrement dit si l'on a

$$
\lim _{y \rightarrow \infty} \sum_{P(n) \leqslant y} \alpha_{n}=\sum_{n=1}^{\infty} \alpha_{n} .
$$

Il est à noter qu'une série $\sum_{P(n) \leqslant y} \alpha_{n}$ est convergente dès que $\alpha_{n} \ll 1 / n^{c}$ avec $c>0$.

Considérons les $P$-sommes partielles de la série $(1 \cdot 2)$, soit

$$
B(\vartheta ; y):=-\sum_{P(n) \leqslant y} \frac{\sin (2 \pi n \vartheta)}{\pi n} .
$$

La remarque fondamentale, qui justifie l'introduction du procédé de $P$-sommation dans cette étude, est que le $P$-analogue de $\left(D_{\vartheta}\right)$

$$
\sum_{P(m) \leqslant y} \frac{f(m)}{\pi m} \sin (2 \pi m \vartheta)+\sum_{P(n) \leqslant y} \frac{g(n)}{n} B(n \vartheta ; y)=0 \quad(y \geqslant 2)
$$

est trivialement vérifié dès que

$$
\sum_{P(n) \leqslant y}|g(n)| / n<\infty .
$$

Introduisant les $P$-sommes partielles de la série $(1 \cdot 8)$, soit

$$
\nabla_{f}(\vartheta ; y):=\sum_{P(m) \leqslant y} \frac{f(m)}{\pi m} \sin (2 \pi m \vartheta)+\sum_{P(n) \leqslant y} \frac{g(n)}{n} B(n \vartheta),
$$

et les «défauts de $P$-régularité» des $P$-sommes correspondant aux séries $U(f ; \vartheta)$ et $V(f ; \vartheta)$, soit

$$
U_{f}(\vartheta ; y):=\sum_{\substack{P(m) \leqslant y \\ m>y}} \frac{f(m)}{\pi m} \sin (2 \pi m \vartheta), \quad V_{g}(\vartheta ; y):=\sum_{\substack{P(n) \leqslant y \\ n>y}} \frac{g(n)}{n} B(n \vartheta),{ }^{(5)}
$$

nous déduisons trivialement de $(1 \cdot 15)$ une autre identité pour $\Delta_{f}(\vartheta ; y)$ :

$$
\Delta_{f}(\vartheta ; y)=\nabla_{f}(\vartheta ; y)-U_{f}(\vartheta ; y)-V_{g}(\vartheta ; y) \quad(y \geqslant 2) .
$$

Cette formule est la base de notre approche. Elle implique en particulier que $\left(D_{\vartheta}\right)$ est réalisée dès que les deux séries sont $P$-régulières et

$$
\nabla_{f}(\vartheta ; y)=o(1) \quad(y \rightarrow \infty) .
$$

Cela conduit naturellement à la définition suivante.

5. De sorte que, sous réserve de convergence ordinaire, les séries de $\left(D_{\vartheta}\right)$ sont $P$-régulières si, et seulement si, $U_{f}(\vartheta ; y)=o(1)$ ou $V_{g}(\vartheta ; y)=o(1)$, respectivement. 
Définition 1.3. On dit qu'un couple $(f, g)$ de fonctions arithmétiques liées par la relation (1.3) est $\vartheta$-adapté si l'on a (1.14) pour tout $y \geqslant 2$ et si la relation (1.17) est satisfaite.

Il est utile de garder à l'esprit que, pour un couple $(f, g) \vartheta$-adapté, la $P$-convergence de l'une des deux séries $U(f ; \vartheta)$ et $V(g ; \vartheta)$ implique celle de l'autre, les $P$-sommes étant alors nécessairement opposées.

À fins de référence ultérieure nous récapitulons dans la proposition suivante les conditions suffisantes usuelles de validité de $\left(D_{\vartheta}\right)$ pour un couple $\vartheta$-adapté.

Proposition 1.4. Soit $(f, g)$ un couple $\vartheta$-adapté. On a $(f, g) \in D_{\vartheta}$ si l'une au moins des conditions suivantes est réalisée :

(a) la série $U(f ; \vartheta)$ converge et $U_{f}(\vartheta ; y)=o(1), V_{g}(\vartheta ; y)=o(1)$ lorsque $y \rightarrow \infty$;

(b) la série $V(g ; \vartheta)$ converge et $U_{f}(\vartheta ; y)=o(1), V_{g}(\vartheta ; y)=o(1)$ lorsque $y \rightarrow \infty$;

(c) les deux séries $U(f ; \vartheta)$ et $V(g ; \vartheta)$ sont convergentes et l'on a

$$
\liminf _{y \rightarrow \infty}\left|V_{g}(\vartheta ; y)\right|+\left|U_{f}(\vartheta ; y)\right|=0 .
$$

De plus, sous les conditions (a) ou (b), les deux séries de $\left(D_{\vartheta}\right)$ sont $P$-régulières.

Bien entendu, si $(f, g)$ est $\vartheta$-adapté, les séries de $\left(D_{\vartheta}\right)$ sont simultanément $P$ convergentes ou simultanément $P$-divergentes. Il serait intéressant de savoir si elles sont nécessairement simultanément $P$-régulières. Dans le cas contraire, il faudrait encore déterminer s'il peut se produire qu'une seule des deux séries soit convergente au sens ordinaire ou si, lorsque les deux séries sont à la fois $P$-convergentes et convergentes, la $P$-régularité de l'une implique celle de l'autre. Nous verrons plus loin que les deux séries associées à un couple $\vartheta$-adapté peuvent être $P$-convergentes alors que l'une au moins est divergente au sens usuel.

D'après [14], on a pour tout $\vartheta \in \mathbb{R}$

$$
\nabla_{\mathbf{1}}(\vartheta ; y)=\sum_{P(m) \leqslant y} \frac{\sin (2 \pi m \vartheta)}{\pi m}+B(\vartheta)=o(1) \quad(y \rightarrow \infty),
$$

ce qui est une reformulation de la $P$-régularité de la série $B(\vartheta)$ pour tout nombre réel $\vartheta$, puisque

$$
B(\vartheta ; y)=B(\vartheta)-\nabla_{\mathbf{1}}(\vartheta ; y) .
$$

Le $P$-analogue du membre de gauche de $(1 \cdot 7)$ est

$$
\nabla(\vartheta ; y):=\nabla_{\delta}(\vartheta ; y)=\frac{\sin (2 \pi \vartheta)}{\pi}+\sum_{P(n) \leqslant y} \frac{\mu(n)}{n} B(n \vartheta) .
$$

Nous verrons plus loin (Théorème 2.1 ) que cette quantité est $\ll 1 / \log y$ uniformément en $\vartheta$, avec même une majoration meilleure lorsque $\vartheta$ est irrationnel. 
Lorsque $f$ et $g$ sont liées par (1·3), (1·13) implique

$$
\nabla_{f}(\vartheta ; y)=\sum_{P(m) \leqslant y} \frac{f(m)}{m} \nabla(m \vartheta ; y)=\sum_{P(n) \leqslant y} \frac{g(n)}{n} \nabla_{\mathbf{1}}(n \vartheta ; y) .
$$

Les conditions (a), (b), (c) de la Proposition 1.4, liées à la $P$-régularité, sont fines par nature. En revanche, il apparait au vu de (1.22) que la $\vartheta$-adaptation est une forme en moyenne de l'une ou l'autre des relations $\nabla(\vartheta ; y)=o(1), \nabla_{\mathbf{1}}(\vartheta ; y)=o(1)$, que l'on sait, d'après respectivement $(1 \cdot 19)$ et $(2 \cdot 3)$ infra, être satisfaites pour tout $\vartheta$. Il est donc naturel d'espérer que la $\vartheta$-adaptation a lieu sous des conditions relativement générales : c'est effectivement ce que nous établissons au paragraphe 3.

\section{Estimations fondamentales : $\nabla(\vartheta ; y)$ et $\boldsymbol{B}(\vartheta ; \boldsymbol{y})$}

Nous donnons dans ce paragraphe des évaluations quantitatives de $\nabla(\vartheta ; y)$ et $B(\vartheta ; y)$ en fonction de $y$ et des approximations rationnelles de $\vartheta$.

Le résultat suivant, relatif à $\nabla(\vartheta ; y)$, constitue une version effective de la $P$ régularité de la série $V(\mu ; \vartheta)$. Ici et dans la suite, nous désignons par $\tau(q)$ le nombre des diviseurs d'un entier naturel $q$. Conformément à la tradition, nous notons $\varphi$ la fonction indicatrice d'Euler.

Plusieurs de nos résultats sont exprimés en fonction des bonnes approximations rationnelles d'un nombre réel $\vartheta$. Posons, selon l'usage,

$$
\|\vartheta\|:=\min _{n \in \mathbb{Z}}|\vartheta-n| \quad(\vartheta \in \mathbb{R}) .
$$

Pour $\vartheta \in \mathbb{R}, Q \in \mathbb{N}^{*}$ on a, en vertu du théorème de Dirichlet,

$$
\mu(\vartheta ; Q):=\min _{1 \leqslant m \leqslant Q}\|m \vartheta\| \leqslant 1 / Q .
$$

Nous utilisons systématiquement la notation

$$
q(\vartheta ; Q):=\min \{q: 1 \leqslant q \leqslant Q,\|q \vartheta\|=\mu(\vartheta ; Q)\} .
$$

Par commodité d'écriture, nous étendons la définition de $t \mapsto q(\vartheta ; t)$ à $[1, \infty[$ en posant $q(\vartheta ; t):=q(\vartheta ;[t])$. Les $q(\vartheta ; t)$ décrivent l'ensemble

$$
\left\{q:\|q \vartheta\|<\min _{1 \leqslant r<q}\|r \vartheta\|\right\}
$$


Théorème 2.1. Soit $A>0$. On a uniformément sous les conditions $y \geqslant 2$, $Q_{y}:=y /(\log y)^{5 A+20}, \vartheta \in \mathbb{R}, q:=q\left(\vartheta ; Q_{y}\right)$,

$$
\nabla(\vartheta ; y) \ll \frac{q \tau(q)^{2}}{\varphi(q)^{2} \log y}+\frac{1}{(\log y)^{A}} .
$$

De plus, on a uniformément pour $y \geqslant 2, a \in \mathbb{Z}, q \in \mathbb{N}, 1 \leqslant q \leqslant Q_{y}$,

$$
\nabla(a / q ; y) \ll \frac{1}{(\log y)^{A}} .
$$

La puissance du logarithme dans le premier terme du membre de droite de $(2 \cdot 3)$ est optimale compte tenu de l'uniformité imposée : il résulte en effet immédiatement de $(1 \cdot 21)$ que

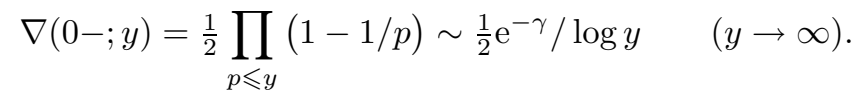

Cette formule est étendue et précisée au Lemme 9.4. Il est intéressant, à ce propos, de noter que l'étude des variations de $\nabla(\vartheta ; y)$ se confond essentiellement avec celle des discontinuités : en tout point $\vartheta$ où la fonction $\nabla(\vartheta ; y)$ est dérivable, c'est-à-dire lorsque $\vartheta \prod_{p \leqslant y} p \notin \mathbb{Z}$, la somme en $n$ de $(1 \cdot 21)$ est de dérivée nulle, de sorte que $\nabla^{\prime}(\vartheta ; y)=2 \cos (2 \pi \vartheta)$.

Le second résultat de ce paragraphe est une version quantitative uniforme en $\vartheta$ de $(1 \cdot 19)$ — et donc une mesure effective de la convergence de $B(\vartheta ; y)$.

Nous utilisons les notations

$$
u_{t, y}:=\log t / \log y, \quad u:=u_{x, y}, \quad u_{\vartheta, y}^{*}:=u_{1 /\|\vartheta\|, y} .
$$

Conformément à l'usage, nous notons $\varrho(v)$ la fonction de Dickman, définie comme l'unique solution continue à droite sur $\mathbb{R}$ tout entier de l'équation différentielle aux différences

$$
v \varrho^{\prime}(v)+\varrho(v-1)=0 \quad(v>1)
$$

avec les conditions initiales $\varrho(v)=0(v<0)$ et $\varrho(v)=1(0 \leqslant v \leqslant 1)$. Des informations complémentaires sur la fonction de Dickman sont disponibles, par exemple, aux paragraphes III.5.3-4 de [29].

Théorème 2.2. Soit $A>0$. On a uniformément sous les conditions $y \geqslant 2$, $Q_{y}:=y /(\log y)^{4 A+20}, \vartheta \in \mathbb{R}, q:=q\left(\vartheta ; Q_{y}\right)$,

$(2 \cdot 6) B(\vartheta ; y)=\varrho\left(u_{\vartheta, y}^{*}\right) B(\vartheta)+O\left(\frac{2^{\omega(q)}(\log q)^{2} \varrho\left(u_{\vartheta, y}^{*}\right) \log \left(u_{\vartheta, y}^{*}+2\right)}{\varphi(q) \log y}+\frac{1}{(\log y)^{A}}\right)$.

En particulier, on a

$$
\nabla_{\mathbf{1}}(\vartheta ; y) \ll \frac{2^{\omega(q)}(\log q)^{2}}{\varphi(q) \log y}+\frac{1}{(\log y)^{A}}
$$

dès que $\|\vartheta\| \geqslant 1 / y$. 
On déduit immédiatement de $(2 \cdot 7)$ que

$$
B(\vartheta ; y)=B(\vartheta)+O(1 / \log y) \quad(\|\vartheta\| \geqslant 1 / y)
$$

Lorsque $\vartheta$ est très proche d'un entier, l'estimation $(2 \cdot 8)$ n'est plus valable, ${ }^{(6)}$ et $B(\vartheta ; y)$ n'approche pas $B(\vartheta)$. Cependant, contrairement au cas de la convergence usuelle des séries, la $P$-convergence permet d'éviter le phénomène de Gibbs (cf. [35], chapitre 8) : la formule $(2 \cdot 6)$ fournit ainsi

$$
\lim _{y \rightarrow \infty} \sup _{\vartheta \in \mathbb{R}} B(\vartheta ; y)=\frac{1}{2}=\sup _{\vartheta \in \mathbb{R}} B(\vartheta) .
$$

Nous généralisons ce résultat au paragraphe 5 .

\section{Critères de $\boldsymbol{\vartheta}$-adaptation}

Pour $\alpha>0$, nous désignons par $\mathcal{L}^{\alpha}\left(\mathbb{N}^{*}\right)$, ou plus simplement $\mathcal{L}^{\alpha}$, la classe des fonctions arithmétiques $h$ satisfaisant à

$$
\|h\|_{\alpha}:=\left\{\limsup _{x \rightarrow \infty} \frac{1}{x} \sum_{n \leqslant x}|h(n)|^{\alpha}\right\}^{1 / \alpha}<\infty .
$$

Le Théorème 2.1 permet d'obtenir une condition suffisante très générale pour qu'un couple $(f, g)$ soit $\vartheta$-adapté.

Théorème 3.1. Soient $\alpha>1, \vartheta \in \mathbb{R}$, et $(f, g)$ un couple de fonctions arithmétiques satisfaisant (1·3). Si $f \in \mathcal{L}^{\alpha}$, alors $(f, g)$ est $\vartheta$-adapté.

Une autre conséquence, beaucoup plus simple, de la majoration $(2 \cdot 3)$ du Théorème 2.1 et de la formule $(1 \cdot 22)$ fait l'objet de la première assertion de l'énoncé suivant.

Théorème 3.2. Soient $\vartheta \in \mathbb{R}$ et $(f, g)$ un couple de fonctions arithmétiques satisfaisant (1·3). Alors $(f, g)$ est $\vartheta$-adapté dès que

$$
\frac{1}{\log y} \sum_{P(m) \leqslant y} \frac{|f(m)|}{m}=o(1) \quad(y \rightarrow \infty) .
$$

De plus, il existe une infinité de triplets $(\vartheta, f, g)$ tels que

$$
\frac{1}{\log y} \sum_{P(m) \leqslant y} \frac{|f(m)|}{m} \ll 1 \quad(y \geqslant 2)
$$

alors que $(f, g)$ n'est pas $\vartheta$-adapté.

6. Le prolongement par continuité fournit $\varrho\left(u_{\vartheta, y}^{*}\right)=0$ lorsque $\vartheta \in \mathbb{Z}$. 
Le Théorème 3.1 permet aussi d'exhiber, conformément à ce qui a été annoncé au paragraphe 1 , des couples $(f, g) \vartheta$-adaptés pour lesquels les séries $U(f ; \vartheta)$ et $V(g ; \vartheta)$ sont toutes deux $P$-convergentes — donc, par $(1 \cdot 15)$ et $(1 \cdot 17)$, vers des limites opposées — mais tels que $U(f ; \vartheta)$ soit divergente au sens ordinaire.

Posons

$$
S(x, y):=\{n \leqslant x: P(n) \leqslant y\} .
$$

La construction de notre contre-exemple repose sur un résultat qui découle facilement des estimations de [14] pour la quantité

$$
E(x, y ; \vartheta):=\sum_{n \in S(x, y)} \mathrm{e}(n \vartheta)
$$

et que nous énonçons ici formellement à fins de référence ultérieure. Ici et dans la suite nous utilisons la notation classique

$$
\mathrm{e}(u):=\mathrm{e}^{2 \pi i u} \quad(u \in \mathbb{R})
$$

Proposition 3.3. Soient $\vartheta, \tau$ des nombres réels. Alors la série

$$
\sum_{n \geqslant 1} \frac{\mathrm{e}(n \vartheta)}{n^{1+i \tau}}
$$

est $P$-régulière si, et seulement si, l'une des deux conditions suivantes est réalisée :

(a) $\tau=0$ et, pour toute puissance de nombre premier $p^{\nu}, p^{\nu} \vartheta \notin \mathbb{Z}$;

(b) $\tau \neq 0$ et $\vartheta \notin \mathbb{Z}$.

Démonstration. Le cas $\tau=0$ a été traité dans [14] (théorème 11). Lorsque $\tau \neq 0$ et $\vartheta \in \mathbb{Z}$, il résulte du théorème des nombres premiers que la série (non convergente) (3.4) est $P$-convergente, de $P$-somme $\zeta(1+i \tau)$. Lorsque $\tau \neq 0$ et $\vartheta \notin \mathbb{Z}$, les calculs sont en fait identiques à ceux de de la démonstration du théorème 11 de [14], à ceci près que, dans le cas $\vartheta \in \mathbb{Q}$, la $P$-régularité découle du lemme de RiemannLebesgue, dont l'application est permise par la non nullité de $\tau$. Nous omettons les détails.

Nous sommes maintenant en mesure de décrire le contre-exemple annoncé.

Soient $\tau \in \mathbb{R}^{*}, \alpha \in \mathbb{R} \backslash \frac{1}{2} \mathbb{Z}$. Considérons la fonction arithmétique $f=f_{\alpha, \tau}$ définie par $f(m):=\sin (2 \pi m \alpha) / m^{i \tau}$. Ainsi qu'il a été vu plus haut, la série $\sum_{m=1}^{\infty} 1 / m^{1+i \tau}$ est $P$-convergente et non convergente alors que la série $\sum_{m=1}^{\infty} \cos (2 \pi m \vartheta) / m^{1+i \tau}$ est $P$-régulière pour $\vartheta \in \mathbb{R} \backslash \mathbb{Z}$. Grâce à la relation

$$
\frac{f(m) \sin (2 \pi m \vartheta)}{m}=\frac{\cos \{2 \pi(\vartheta-\alpha) m\}-\cos \{2 \pi(\vartheta+\alpha) m\}}{2 m^{1+i \tau}},
$$

nous voyons donc que la série $U(f ; \vartheta)$ est $P$-régulière si $\vartheta \not \equiv \pm \alpha(\bmod 1)$ alors qu'elle est $P$-convergente, mais divergente au sens ordinaire, si $\vartheta \equiv \pm \alpha(\bmod 1)$. Comme $f$ est bornée, le Théorème 3.1 implique immédiatement, dans ce dernier cas, que la série $V(g ; \vartheta)$ est également $P$-convergente.

Lorsque $\vartheta$ est rationnel, nous pouvons établir un critère de $\vartheta$-adaptation beaucoup plus large. 
Théorème 3.4. Soient $\vartheta \in \mathbb{Q}$ et $(f, g)$ un couple de fonctions arithmétiques liées par la relation (1.3). S'il existe une constante $A>0$ telle que l'une ou l'autre des conditions

$$
\sum_{P(m) \leqslant y} \frac{|f(m)|}{m} \ll(\log y)^{A}, \quad \sum_{P(m) \leqslant y} \frac{|g(m)|}{m} \ll(\log y)^{A}
$$

soit satisfaite, alors $(f, g)$ est $\vartheta$-adapté.

Remarque. L'emploi d'un argument sensiblement plus sophistiqué permettrait de remplacer dans cet énoncé la majoration $(\log y)^{A}$ par $\exp \{c \sqrt{\log y}\}$ pour une constante positive convenable $c$.

Lorsque $g$ est bornée, uniformément ou en moyenne, le Théorème 2.2 permet d'obtenir des conditions suffisantes de $\vartheta$-adaptation portant sur les dénominateurs des réduites de $\vartheta$.

Théorème 3.5. Soit $\vartheta \in \mathbb{R} \backslash \mathbb{Q}$. Si $\left\{q_{m}\right\}_{m=1}^{\infty}$ est la suite des dénominateurs des réduites de $\vartheta$ et si $(f, g)$ est un couple de fonctions arithmétiques satisfaisant $(1 \cdot 3)$, alors $(f, g)$ est $\vartheta$-adapté dès que l'une des conditions suivantes est réalisée :

(a) $g$ est bornée, et l'on a $\log q_{m+1}=o\left(q_{m}\right)(m \rightarrow \infty)$;

(b) il existe $\alpha>1$ et $\beta<1-1 / \alpha$ tels que $g \in \mathcal{L}^{\alpha}$ et $\log q_{m+1} \ll q_{m}^{\beta}(m \geqslant 1)$.

Lorsque $g=\mathbf{1}$, la condition (a) est également nécessaire.

\section{Critères de validité pour la relation $\left(D_{\vartheta}\right)$}

Posons

$$
B^{*}(\vartheta ; y):=-\sum_{1 \leqslant n \leqslant y} \frac{\sin (2 \pi n \vartheta)}{\pi n}
$$

de sorte que l'on a classiquement

$$
B(\vartheta)=B^{*}(\vartheta ; y)+O\left(\frac{1}{1+y\|\vartheta\|}\right)
$$

La relation $(4 \cdot 2)$ est une forme effective de l'assertion $(\mathbf{1}, \boldsymbol{\delta}) \in D_{\vartheta}$ et l'on peut en déduire aisément que $(f, g) \in D_{\vartheta}$ sous la condition

$$
\sum_{n \geqslant 1} \frac{|g(n)|}{n}<\infty
$$

Dans [8], Davenport obtient le résultat pour $f=\mu^{2}$; la fonction $g$ est alors définie par $g(n)=\mu(m)$ si $n=m^{2}, g(n)=0$ si $n$ n'est pas un carré. Pour établir le cas général, nous appliquons $(4 \cdot 2)$ avec $n \vartheta$ et $y / n$ à la place de $\vartheta$ et $y$. Multipliant par 
$g(n) / n$ et sommant sur l'ensemble de tous les entiers $n \leqslant y$ tels que $\|n \vartheta\| \neq 0$, nous obtenons après regroupement dans le membre de droite

$$
\sum_{n \leqslant y} \frac{g(n)}{n} B(n \vartheta)+\sum_{m \leqslant y} \frac{f(m) \sin (2 \pi m \vartheta)}{\pi m} \ll \sum_{n \leqslant y,\|n \vartheta\| \neq 0} \frac{|g(n)|}{n+y\|n \vartheta\|} .
$$

Sous l'hypothèse $(4 \cdot 3)$, le majorant tend bien vers 0 lorsque $y \rightarrow \infty$.

Dans la nouvelle approche du problème explorée ici, la relation (2.7) joue le rôle de $(4 \cdot 2)$ et il n'est pas du tout évident a priori, même en faisant simultanément appel à ces deux relations, que le résultat précédent puisse être retrouvé : il est en effet théoriquement possible que $\Delta_{f}(\vartheta ; y)$ tende vers 0 lorsque $y \rightarrow \infty$ alors qu'il n'en va pas ainsi pour l'un au moins des termes du membre de droite de (1·16). Nous allons voir cependant que la méthode passe allègrement ce premier test, fournissant en fait un renseignement supplémentaire spécifique.

Théorème 4.1. Soit $g$ une fonction arithmétique satisfaisant à (4·3), et $f:=1 * g$. On a $(f, g) \in D_{\vartheta}$ pour tout $\vartheta \in \mathbb{R}$ et les séries $U(f ; \vartheta)$ et $V(g ; \vartheta)$ sont $P$-régulières.

Démonstration. Utilisons le critère (b) de la Proposition 1.4. La $\vartheta$-adaptation du couple $(f, g)$ découle de $(2 \cdot 7)$ et de la seconde expression $(1 \cdot 22)$ pour $\nabla_{f}(\vartheta ; y)$. La $P$-régularité de la série $V(g ; \vartheta)$ résulte immédiatement de $(4 \cdot 3)$.

Il reste à montrer que

$$
U_{f}(\vartheta ; y)=o(1) \quad(y \rightarrow \infty) .
$$

Une interversion de sommations fournit, avec la notation (4·1),

$$
U_{f}(\vartheta ; y)=\sum_{n \leqslant y} \frac{g(n)}{n}\left\{B^{*}\left(n \vartheta ; \frac{y}{n}\right)-B(n \vartheta ; y)\right\}
$$

Par $(4 \cdot 2)$ et $(2 \cdot 7)$, l'expression entre accolades est uniformément bornée en $\vartheta$ et est $\ll n /(n+\log y)$ lorsque, disons, $\|n \vartheta\|>1 / \sqrt{y}$. Il suit

$$
U_{f}(\vartheta ; y) \ll \sum_{P(n) \leqslant y} \frac{|g(n)|}{n+\log y}+\sum_{\substack{P(n) \leqslant y \\ 0<\|n \vartheta\| \leqslant 1 / \sqrt{y}}} \frac{|g(n)|}{n} .
$$

Compte tenu de l'hypothèse $(4 \cdot 3)$, le théorème de Lebesgue implique donc $(4 \cdot 5)$.

Le résultat suivant établit qu'en un certain sens le Théorème 4.1 est optimal.

Théorème 4.2. Pour toute fonction $\xi$ positive telle que $\lim _{y \rightarrow+\infty} \xi(y)=\infty$, il existe une fonction arithmétique $g$ satisfaisant à $\sum_{P(n) \leqslant y}|g(n)| / n \leqslant \xi(y)$ et un nombre réel $\vartheta$ tels que :

(a) la série $V(g ; \vartheta)$ est divergente et $P$-divergente; 
(b) le couple $(f, g)$ avec $f=\mathbf{1} * g$ n'est pas $\vartheta$-adapté.

Le fait que la majoration $(2 \cdot 7)$, qui est le $P$-analogue de $(4 \cdot 2)$, fournisse la même condition taubérienne pour la validité de $\left(D_{\vartheta}\right)$ montre que l'approche du problème via la $P$-sommation n'induit pas de perte systématique. Nous verrons qu'elle conduit en fait à des résultats nouveaux, non seulement en exploitant le $P$-analogue $(2 \cdot 3)$ de $(1 \cdot 7)$ — qui est la version duale de $(4 \cdot 2)$ —, mais aussi en employant directement $(2 \cdot 7)$, qui se révèle dans certains cas plus pertinent que $(4 \cdot 2)$.

Le résultat suivant est un exemple d'une telle situation. Nous y établissons que $(\log , \Lambda) \in D_{\vartheta}$ pour tout nombre réel $\vartheta .^{(7)}$ On peut aisément vérifier qu'avec ce choix des fonctions $f$ et $g$ l'estimation $(4 \cdot 4)$ ne permet pas de conclure. ${ }^{(8)}$

Théorème 4.3. Pour tout $\vartheta \in \mathbb{R}$, on a

$$
\sum_{m=1}^{\infty} \frac{\log m}{\pi m} \sin (2 \pi m \vartheta)+\sum_{n=1}^{\infty} \frac{\Lambda(n)}{n} B(n \vartheta)=0
$$

et les deux séries sont P-régulières.

Dans [7], Davenport a prouvé que (4.7) a lieu pour un ensemble de valeurs de $\vartheta \in \mathbb{T}$ de mesure 1 , contenant les rationnels. La validité inconditionnelle de (4.7) a été annoncée par Segal dans [24] mais sa preuve n'est pas correcte, la justification de l'interversion dans la formule (4) de [24] étant déficiente. Le résultat est repris tel quel dans [17]. L'approche de Segal, qu'il met en œuvre pour les couples $(f, g)=(\boldsymbol{\delta}, \mu)$ et $(f, g)=(\log , \Lambda)$, repose sur la représentation intégrale

$$
B(z)=-\frac{1}{2 \pi i} \int_{-c-i \infty}^{-c+i \infty} \frac{\zeta(s) z^{s}}{s} \mathrm{~d} s \quad(z>0),
$$

certainement valable pour $c \in] 0, \frac{1}{2}\left[.^{(9)}\right.$ Cette formule peut être facilement établie par déplacement de l'abscisse d'intégration à partir d'une intégrale de Perron sur une droite d'abscisse $>1$. L'intégrale de (4.8) est semi-convergente mais non absolument convergente : l'équation fonctionnelle de $\zeta$ et la formule de Stirling complexe fournissent en effet, pour $s=-c+i \tau$,

$$
\frac{\zeta(s) z^{s}}{s}=\frac{|\tau|^{c-1 / 2} \zeta(1+c-i \tau) \mathrm{e}^{i h_{z}(\tau)}}{i z^{c}(2 \pi)^{c+1 / 2}}\left\{1+O\left(\frac{1}{|\tau|}\right)\right\} \quad(|\tau| \geqslant 1)
$$

7. Conformément à l'usage, nous désignons par $\Lambda$ la fonction de von Mangoldt.

8. En effet,

$$
\sum_{n \leqslant y,\|n \vartheta\| \neq 0} \frac{\Lambda(n)}{n+y\|n \vartheta\|} \geqslant \sum_{y / 2<n \leqslant y} \frac{\Lambda(n)}{2 n}=\frac{1}{2} \log 2+o(1) .
$$

9. Segal affirme que $(4 \cdot 8)$ est valable pour $0<c<1$. L'intégrale de $(4 \cdot 8)$ ne converge pas pour $\frac{1}{2}<c<1$. 
avec $h_{z}(\tau):=\tau \log (2 \pi z /|\tau|)+\tau-\frac{3}{4} \pi \operatorname{sgn}(\tau)$. Il est à noter que la discontinuité de $B$ en $z=0$ interdit une approximation uniforme de $B(z)$ par troncature de l'intégrale de (4.8). En appliquant (4.8) à $z=n \vartheta$ pour $n \in \mathbb{N}^{*}$ et en sommant sur $n \leqslant x$, on obtient

$$
\sum_{n \leqslant x} \frac{g(n)}{n} B(n \vartheta)=-\frac{1}{2 \pi i} \int_{c-i \infty}^{c+i \infty} \frac{\zeta(-s)}{s \vartheta^{s}} \sum_{n \leqslant x} \frac{g(n)}{n^{s+1}} \mathrm{~d} s .
$$

Un passage à la limite sous le signe d'intégration et l'application de l'équation fonctionnelle de $\zeta$ suivi de la formule d'inversion de Mellin fournissent formellement $(f, g) \in D_{\vartheta}$ lorsque, par exemple, $g=\mu$. Cependant, contrairement à ce qui est annoncé dans [24], la convergence absolue de $\sum_{n} g(n) / n^{s+1}$ ne suffit pas à justifier le procédé puisque l'intégrande du second membre de (4-10) n'est pas dominé par une fonction intégrable.

Le développement en série de Fourier de $\log \Gamma(\vartheta)$ obtenu par Kummer $[21]^{(10)}$ permet d'exprimer la somme des séries de (4.7) en termes de fonctions classiques. On a pour tout $\vartheta \in \mathbb{R} \backslash \mathbb{Z}$

$$
\sum_{m \geqslant 1} \frac{\log m}{\pi m} \sin (2 \pi m \vartheta)=\log \left(\frac{\Gamma(\langle\vartheta\rangle) \sqrt{|\sin (\pi \vartheta)|}}{\pi \sqrt{2}}\right)+(\gamma+\log 2 \pi)\langle\vartheta\rangle-\frac{1}{2} \gamma,
$$

où $\gamma$ est la constante d'Euler.

Le Théorème 4.3 n'est pas transposable au couple $(f, g)=(\tau, \mathbf{1})$ : ainsi que l'a remarqué Chowla [6], il existe des réels $\vartheta$ tels que la série $V(\mathbf{1} ; \vartheta)$ diverge. ${ }^{(11)}$ Le Théorème 3.5 nous permet de caractériser complètement les nombres réels $\vartheta$ tels que $(\tau, \mathbf{1}) \in D_{\vartheta}$.

Théorème 4.4. On a

$$
\sum_{m \geqslant 1} \frac{\tau(m)}{\pi m} \sin (2 \pi m \vartheta)+\sum_{n \geqslant 1} \frac{B(n \vartheta)}{n}=0
$$

pour tout $\vartheta \in \mathbb{Q}$. Pour $\vartheta \in \mathbb{R} \backslash \mathbb{Q}$, la relation (4.11) est valide si, et seulement si, la série

$$
\sum_{m \geqslant 1}(-1)^{m} \frac{\log q_{m+1}}{q_{m}}
$$

converge, où $\left\{q_{m}\right\}_{m \geqslant 1}$ désigne la suite des dénominateurs des réduites de $\vartheta$. Lorsque la série (4·12) diverge, il en va de même des deux séries de (4·11).

10. Voir Whittaker et Watson [32] §12.31.

11. Un calcul élémentaire permet en effet de montrer que l'on a, pour $y \geqslant q-1$,

$$
\sum_{n \leqslant y} \frac{B(n / q)}{n}=-\frac{1}{2} \sum_{1 \leqslant b<q} \frac{1}{b}+O(1)=-\frac{1}{2} \log q+O(1) .
$$

Cela permet de construire des irrationnels $\vartheta$ pour lesquels la série $V(\mathbf{1} ; \vartheta)$ diverge. 
L'équivalence des convergences de $(4 \cdot 12)$ et de la première série $(4 \cdot 11)$ n'est pas nouvelle : elle été établie, avec une méthode totalement différente et sensiblement plus compliquée, par Wilton [33]. Par ailleurs, Wilton ne considère pas la question de l'identité $(4 \cdot 11)$.

Les nombres de Bruno — voir [34] —, qui sont définis par la condition

$$
\sum_{m \geqslant 1}\left(\log q_{m+1}\right) / q_{m}<\infty
$$

satisfont $(4 \cdot 12)$. Ils constituent donc, en vertu du Théorème 4.4, des exemples non triviaux de nombres irrationnels pour lesquels $(4 \cdot 11)$ a lieu.

On peut, plus généralement, poser la question de la validité de $\left(D_{\vartheta}\right)$ lorsque $f=\tau^{\alpha}$ avec $\alpha \in \mathbb{R}$. Le Théorème 1.1 fournit une réponse affirmative pour tout $\vartheta \in \mathbb{R}$ lorsque $\alpha<0$. La relation $\left(D_{\vartheta}\right)$ a encore lieu lorsque $\alpha=0$, autrement dit $f=1$ : elle coïncide alors avec le développement en série de Fourier $(1 \cdot 2)$ de la fonction $B$. En revanche, pour chaque $\alpha>0$, on peut construire des nombres réels $\vartheta$ invalidant $\left(D_{\vartheta}\right)$ en imposant la divergence d'une série analogue à $(4 \cdot 12)$, qui correspond au cas $\alpha=1$.

Les estimations de $\sum_{n \in S(x, y)} \mu(n) B(n \vartheta)$ établies au cours de la preuve du Théorème 2.1 fournissent des cas de validité pour $\left(D_{\vartheta}\right)$ fondamentalement distincts de ceux qui, à l'instar de (4·7), sont déduits des évaluations du Théorème 2.2.

Théorème 4.5. Pour tout $\vartheta \in \mathbb{R}$, on a

$$
\sum_{m \geqslant 1} \frac{\Lambda(m)}{\pi m} \sin (2 \pi m \vartheta)-\sum_{n \geqslant 1} \frac{\mu(n) \log n}{n} B(n \vartheta)=0
$$

et ces deux séries sont $P$-régulières.

De plus, la convergence des deux séries de (4-13) est uniformément bornée.

Comme nous l'avons remarqué dans l'introduction, un argument simple permet de déduire de ce résultat que $(\Lambda * h,-(\mu \log ) * h) \in D_{\vartheta}$ pour tout $\vartheta \in \mathbb{R}$ si la fonction $h$ satisfait (1.4). Il est à noter que le Théorème 4.3, qui correspond au cas $h=\mathbf{1}{ }^{(12)}$ représente un cas de validité essentiellement différent.

Notre approche est également pertinente lorsque les fonctions $f$ et $g$ sont multiplicatives. Pour chaque valeur du paramètre réel $A \geqslant 1$, nous désignons par $\mathcal{F}_{A}$ la classe des fonctions arithmétiques multiplicatives $f$ telles que

$$
\sup _{p}|f(p)| \leqslant A, \quad \text { et } \sum_{m \leqslant t}|f(m)|^{2} \leqslant A^{2} t \quad\left(t \in \mathbb{R}^{+}\right) .
$$

Il est clair que $\mathcal{F}_{1}$ contient l'ensemble $\mathcal{M}_{1}$ des fonctions multiplicatives à valeurs dans le disque unité fermé.

12. On a classiquement $\Lambda=-(\mu \log ) * 1$. 
Théorème 4.6. Soient $A>0,(f, g) \in \mathcal{F}_{A} \times \mathcal{F}_{A}$ un couple de fonctions arithmétiques satisfaisant (1.3) et $\vartheta \in \mathbb{R} \backslash \mathbb{Q}$. Alors on a $(f, g) \in D_{\vartheta}$ dès que les séries $U(f ; \vartheta)$ et $V(g ; \vartheta)$ sont convergentes.

Soit $\mathcal{F}_{A}^{*}$ le sous-ensemble de $\mathcal{F}_{A}$ constitué des fonctions multiplicatives $f$ qui, outre les conditions $(4 \cdot 14)$, satisfont également à

$$
\sup _{p} \sup _{\nu \geqslant 2}\left|f\left(p^{\nu}\right)\right| / p^{\delta \nu / 2} \leqslant A
$$

pour un nombre réel convenable de $[0,1[$. Le résultat suivant fournit un critère pratique de validité de $\left(D_{\vartheta}\right)$ lorsque $f$ et $g$ sont multiplicatives, $g \in \mathcal{F}_{A}^{*}$ et $\vartheta \in \mathbb{Q}$.

Théorème 4.7. Soient $q \in \mathbb{N}^{*}, A>0, g \in \mathcal{F}_{A}^{*}$ et $f:=g * 1$. On suppose d'une part que, pour tout caractère $\chi$ impair ${ }^{(13)}$ de module divisant $q$, on a

$$
p \leqslant(A+1)^{2} \Rightarrow \sum_{\nu \geqslant 0} \frac{g\left(p^{\nu}\right) \chi\left(p^{\nu}\right)}{p^{\nu}} \neq 0
$$

et d'autre part que l'une des trois conditions équivalentes suivantes est réalisée :

(a) la série $\sum_{n=1}^{\infty} g(n) \chi(n) / n$ converge pour tout caractère $\chi$ impair dont le module divise $q$;

(b) la série $\sum_{n=1}^{\infty} g(n) \sin (2 \pi b n / q) / n$ converge pour tout $b \in \mathbb{N}$;

(c) la série $V(g ; b / q)=\sum_{n=1}^{\infty} g(n) B(b n / q) / n$ converge pour tout $b \in \mathbb{N}$.

Alors on a $(f, g) \in D_{\vartheta}$ pour tout nombre rationnel $\vartheta=a / q$ avec $a \in \mathbb{Z}$. De plus, les séries $U(f ; a / q)$ et $V(g ; a / q)$ sont $P$-régulières.

Pour $z \in \mathbb{C}$, nous désignons par $\tau_{z}$ la puissance de convolution d'ordre $z$ de la fonction 1 ; autrement dit, $\tau_{z}(n)$ est le coefficient générique de la série de Dirichlet $\zeta(s)^{z}$. Les Théorèmes 4.6 et 4.7 impliquent $\left(\tau_{z+1}, \tau_{z}\right) \in D_{\vartheta}$ pour tout $\vartheta \in \mathbb{R}$ lorsque $|z| \leqslant 1$ et $|z+1| \leqslant 1$. Une étude spécifique permet de s'affranchir de la première restriction.

Théorème 4.8. Soit $z \in \mathbb{C},|z+1| \leqslant 1$. Alors on a, pour tout $\vartheta \in \mathbb{R}$,

$$
\sum_{m \geqslant 1} \frac{\tau_{z+1}(m)}{\pi m} \sin (2 \pi m \vartheta)+\sum_{n \geqslant 1} \frac{\tau_{z}(n)}{n} B(n \vartheta)=0
$$

13. On rappelle qu'un caractère impair est caractérisé par la relation $\chi(-1)=-1$. 


\section{Sur l'absence de phénomène de Gibbs}

Soit $V B_{n}(\mathbb{T})$ la classe des fonctions $F$ qui sont 1-périodiques, à variation bornée sur $\mathbb{T}=\mathbb{R} / \mathbb{Z}$, et normalisées par $F(\vartheta)=\frac{1}{2}\{F(\vartheta+)+F(\vartheta-)\}$ aux points de discontinuité. Toute fonction $F$ de $V B_{n}(\mathbb{T})$ est dérivable presque partout et la décomposition de Radon-Nikodym de la mesure de Stieltjes dF s'écrit

$$
\mathrm{d} F(\vartheta)=F^{\prime}(\vartheta) \mathrm{d} \vartheta+\mathrm{d} \sigma_{F}(\vartheta)
$$

où la partie singulière $\sigma_{F}$ est également à variation bornée sur $\mathbb{T}$ (cf., par exemple, [23], théorème 6.10). Pour $\alpha>1$, désignons par $V B_{n}^{\alpha}(\mathbb{T})$ le sous-espace de $V B_{n}(\mathbb{T})$ constitué des fonctions $F$ dont la dérivée pp $F^{\prime}$ est dans $L^{\alpha}(\mathbb{T})$. Le résultat suivant généralise $(2 \cdot 6)$ aux éléments de $V B_{n}^{\alpha}(\mathbb{T})$ et implique en particulier, pour les fonctions de cet espace, l'absence de phénomène de Gibbs dans la $P$-sommation de Fourier. Dans ce paragraphe, nous notons $a_{n}(F), b_{n}(F)$ les coefficients de Fourier d'une fonction $F$ de $L^{1}(\mathbb{T})$, et nous définissons la $P$-série de Fourier de $F$ par

$$
F(\vartheta ; y):=a_{0}(F)+\sum_{P(n) \leqslant y}\left\{a_{n}(F) \cos (2 \pi n \vartheta)+b_{n}(F) \sin (2 \pi n \vartheta)\right\}
$$

pour toute valeur de $\vartheta$ où cela a un sens. C'est en particulier le cas si $F \in V B_{n}(\mathbb{T})$ puisque $\left|a_{n}(F)\right|+\left|b_{n}(F)\right| \ll 1 / n$, ce qui implique l'absolue convergence de la série $(5 \cdot 2)$ pour chaque $y \geqslant 2$.

Théorème 5.1. Soient $\alpha>1$ et $F \in V B_{n}^{\alpha}(\mathbb{T})$. Il existe une fonction $\varepsilon_{F}(y)$ satisfaisant à $\lim _{y \rightarrow \infty} \varepsilon_{F}(y)=0$ et telle que l'on ait, uniformément pour $\vartheta \in \mathbb{R}$ et $y \geqslant 2$,

$$
F(\vartheta ; y)-F(\vartheta)=\int_{\mathbb{T}}\left\{\varrho\left(u_{v, y}^{*}\right)-1\right\} B(v) \mathrm{d} \sigma_{F}(\vartheta-v)+O\left(\varepsilon_{F}(y)\right) .
$$

En particulier, on a

$$
\lim _{y \rightarrow \infty} F(\vartheta ; y)=F(\vartheta) \quad(\vartheta \in \mathbb{T})
$$

et

$$
\lim _{y \rightarrow \infty} \sup _{\vartheta \in \mathbb{T}} F(\vartheta ; y)=\sup _{\vartheta \in \mathbb{T}} F(\vartheta)
$$

Démonstration. Pour toute fonction $F$ de $V B_{n}(\mathbb{T})$ et tout entier $n \geqslant 1$, on a

$$
\begin{aligned}
a_{n}(F) \cos (2 \pi n \vartheta)+b_{n}(F) \sin (2 \pi n \vartheta) & =2 \int_{\mathbb{T}} \cos (2 \pi n(\vartheta-v)) F(v) \mathrm{d} v \\
& =-\int_{\mathbb{T}} \frac{\sin (2 \pi n(\vartheta-v))}{\pi n} \mathrm{~d} F(v) \\
& =-\int_{\mathbb{T}} \frac{\sin (2 \pi n v)}{\pi n} \mathrm{~d} F(\vartheta-v) .
\end{aligned}
$$


Cette quantité étant trivialement $\ll 1 / n$, on peut sommer pour $P(n) \leqslant y$. On en déduit que l'on a pour tout $y \geqslant 2$

$$
F(\vartheta ; y)=a_{0}(F)+\int_{\mathbb{T}} B(v ; y) \mathrm{d} F(\vartheta-v) .
$$

Par ailleurs, une simple intégration par parties fournit, dès que $F \in V B_{n}(\mathbb{T})$,

$$
F(\vartheta)=a_{0}(F)+\int_{\mathbb{T}} B(v) \mathrm{d} F(\vartheta-v) .
$$

En effectuant la différence de $(5 \cdot 6)$ et $(5 \cdot 7)$, nous obtenons

$$
F(\vartheta ; y)-F(\vartheta)=\int_{\mathbb{T}} \nabla_{\mathbf{1}}(v ; y) \mathrm{d} F(\vartheta-v)=R_{1}(\vartheta ; y)+R_{2}(\vartheta ; y),
$$

disons, où $R_{1}(\vartheta ; y)$ et $R_{2}(\vartheta ; y)$ désignent les contributions respectives à l'intégrale précédente des mesures $F^{\prime}(\vartheta-v) \mathrm{d} v$ et $\mathrm{d} \sigma_{F}(\vartheta-v)$ définies en $(5 \cdot 1)$.

Soient $r:=\min (2, \alpha), s:=r /(r-1)$. Alors $F^{\prime} \in L^{r}(\mathbb{T})$; un résultat classique (cf., par exemple, [18], theorem I.4.7) implique donc

$$
\begin{aligned}
& \sum_{n \geqslant 1} \frac{\left|a_{n}\left(F^{\prime}\right)\right|+\left|b_{n}\left(F^{\prime}\right)\right|}{n} \\
& \quad \leqslant\left(\sum_{n \geqslant 1} \frac{2}{n^{r}}\right)^{1 / r}\left(\sum_{n \geqslant 1}\left\{\left|a_{n}\left(F^{\prime}\right)\right|^{s}+\left|b_{n}\left(F^{\prime}\right)\right|^{s}\right\}\right)^{1 / s}<\infty .
\end{aligned}
$$

On en déduit que

$$
\begin{aligned}
R_{1}(\vartheta ; y) & =\int_{\mathbb{T}} \nabla_{\mathbf{1}}(\vartheta-v ; y) F^{\prime}(v) \mathrm{d} v \\
& =2 \sum_{P(n)>y} \frac{b_{n}\left(F^{\prime}\right) \cos (2 \pi n \vartheta)-a_{n}\left(F^{\prime}\right) \sin (2 \pi n \vartheta)}{\pi n} \ll \eta_{F}(y)
\end{aligned}
$$

avec $\eta_{F}(y):=\sum_{n>y}\left\{\left|a_{n}\left(F^{\prime}\right)\right|+\left|b_{n}\left(F^{\prime}\right)\right|\right\} / n=o(1)(y \rightarrow \infty)$.

L'estimation $(2 \cdot 6)$ fournit

$$
R_{2}(\vartheta ; y)=\int_{\mathbb{T}}\left\{\varrho\left(u_{v, y}^{*}\right)-1\right\} B(v) \mathrm{d} \sigma_{F}(\vartheta-v)+O_{F}(1 / \log y) .
$$

En reportant $(5 \cdot 10)$ et $(5 \cdot 11)$ dans $(5 \cdot 8)$, nous obtenons bien $(5 \cdot 3)$, quitte à choisir $\varepsilon_{F}(y):=\eta_{F}(y)+1 / \log y$.

Comme $\left\{\varrho\left(u_{v, y}^{*}\right)-1\right\} B(v)$ tend simplement vers 0 sur $\mathbb{T}$, la relation (5.3) implique clairement $(5 \cdot 4)$, en vertu du théorème de la convergence dominée. 
Montrons maintenant (5·5). On déduit immédiatement de (5·4) que

$$
\lim _{y \rightarrow \infty} \sup _{\vartheta} F(\vartheta ; y) \geqslant \sup _{\vartheta} F(\vartheta) \text {. }
$$

Il suffit donc de prouver l'inégalité inverse. Nous pouvons supposer sans perte de généralité que $a_{0}(F)=0$. Posons

$$
F_{y}(\vartheta):=\int_{\mathbb{T}} B(v) F^{\prime}(\vartheta-v) \mathrm{d} v+\int_{\mathbb{T}} \varrho\left(u_{v, y}^{*}\right) B(v) \mathrm{d} \sigma_{F}(\vartheta-v)
$$

de sorte que, par $(5 \cdot 3)$ et $(5 \cdot 7)$, on a

$$
\sup _{\vartheta}\left|F(\vartheta ; y)-F_{y}(\vartheta)\right|=o(1) \quad(y \rightarrow \infty) .
$$

Comme l'application $v \mapsto \varrho\left(u_{v, y}^{*}\right) B(v)$ est continue sur $\mathbb{T}, F_{y}(\vartheta)$ est, pour chaque $y$, une fonction continue de $\vartheta$. Elle atteint donc son maximum en un point $\vartheta_{y}$. Quitte à extraire une sous-suite, on peut supposer que

$$
\lim _{y} F_{y}\left(\vartheta_{y}\right)=\lim _{y} \sup _{\vartheta} F\left(\vartheta_{y} ; y\right)=\lim _{y} \sup _{\vartheta} F(\vartheta ; y)
$$

et que $\vartheta_{y}$ tend vers une limite $\vartheta_{0} \in \mathbb{T}$. On peut encore supposer, quitte à changer $F(\vartheta)$ en $F\left(\vartheta-\vartheta_{0}\right)$, que $\vartheta_{0}=0$.

Soit $\delta_{F}:=\sigma_{F}(0+)-\sigma_{F}(0-)$ le saut de $\sigma_{F}$ en 0 . Posons

$$
H(\vartheta):= \begin{cases}0 & \text { si } \vartheta<0 \\ \frac{1}{2} & \text { si } \vartheta=0 \\ 1 & \text { si } \vartheta>0\end{cases}
$$

de sorte que $\widetilde{\sigma}_{F}:=\sigma_{F}-\delta_{F} H$ est continue en 0 . On a pour $\vartheta \in \mathbb{T}$

$(5 \cdot 12) F_{y}(\vartheta)=\int_{\mathbb{T}} B(v) F^{\prime}(\vartheta-v) \mathrm{d} v+\int_{\mathbb{T}} \varrho\left(u_{v, y}^{*}\right) B(v) \mathrm{d} \widetilde{\sigma}_{F}(\vartheta-v)+\delta_{F} \varrho\left(u_{\vartheta, y}^{*}\right) B(\vartheta)$.

La première intégrale est classiquement une fonction continue de $\vartheta$. Donc

$$
\lim _{y \rightarrow \infty} \int_{\mathbb{T}} B(v) F^{\prime}\left(\vartheta_{y}-v\right) \mathrm{d} v=\int_{\mathbb{T}} B(v) F^{\prime}(-v) \mathrm{d} v .
$$

Comme $\varrho\left(u_{v, y}^{*}\right) B(v)$ tend simplement vers $B(v)$ sur $\mathbb{T}$ avec une convergence uniforme sur tout compact de $\mathbb{T}$ ne contenant pas 0 , et comme $\widetilde{\sigma}_{F}$ est continue en 0 , on a

$$
\lim _{y \rightarrow \infty} \int_{\mathbb{T}} \varrho\left(u_{v, y}^{*}\right) B(v) \mathrm{d} \widetilde{\sigma}_{F}\left(\vartheta_{y}-v\right)=\int_{\mathbb{T}} B(v) \mathrm{d} \widetilde{\sigma}_{F}(-v)
$$

Il suit

$$
F_{y}\left(\vartheta_{y}\right) \leqslant \int_{\mathbb{T}} B(v) F^{\prime}(-v) \mathrm{d} v+\int_{\mathbb{T}} B(v) \mathrm{d} \widetilde{\sigma}_{F}(-v)+\frac{1}{2}\left|\delta_{F}\right|+o(1) .
$$

Or, il découle de $(5 \cdot 12)$ que

$$
\max \{F(0+), F(0-)\}=\int_{\mathbb{T}} B(v) F^{\prime}(-v) \mathrm{d} v+\int_{\mathbb{T}} B(v) \mathrm{d} \widetilde{\sigma}_{F}(-v)+\frac{1}{2}\left|\delta_{F}\right| .
$$

On en déduit que $F_{y}\left(\vartheta_{y}\right) \leqslant \sup _{\vartheta} F(\vartheta)+o(1)$. Cela achève la preuve de $(5 \cdot 5)$. 


\section{Sommes d'exponentielles}

\subsection{Préliminaires}

Avec la notation $(3 \cdot 3)$ pour l'ensemble $S(x, y)$, nous posons

$(6 \cdot 1) \quad E_{g}(x, y ; \vartheta):=\sum_{n \in S(x, y)} g(n) \mathrm{e}(\vartheta n), \quad Z_{g}(x, y ; \vartheta):=\sum_{n \in S(x, y)} g(n) \sin (2 \pi \vartheta n)$.

Lorsque $g=\mathbf{1}$, nous omettons l'indice, soit

$$
E(x, y ; \vartheta):=E_{\mathbf{1}}(x, y ; \vartheta), \quad Z(x, y ; \vartheta):=Z_{\mathbf{1}}(x, y ; \vartheta)
$$

La quantité

$$
\Psi(x, y):=|S(x, y)|
$$

constitue la borne triviale de référence pour les quantités (6 1 ) lorsque $|g| \leqslant 1$. Nous aurons souvent l'occasion d'utiliser le résultat uniforme suivant, établi dans [31] (exercice corrigé III.5.6).

Lemme 6.1. On a

$$
\Psi(x, y) \ll x u^{-u}+x^{1 / 4} \ll x \mathrm{e}^{-u / 2} \quad(x \geqslant 2, y \geqslant 2) .
$$

Nos estimations pour les quantités (6-1) sont génériquement exprimées en fonction des bonnes approximations rationnelles de $\vartheta$, dont les dénominateurs sont définis en $(2 \cdot 1)$. Nous aurons ultérieurement l'occasion d'exploiter la minoration suivante de $q(m \vartheta ; Q)(m \geqslant 1)$.

Lemme 6.2. Soient $\vartheta \in \mathbb{R}, Q \in \mathbb{N}^{*}, m \in \mathbb{N}^{*}, q:=q(\vartheta ; Q), q_{m}:=q(m \vartheta ; 2 Q)$. Alors l'une au moins des deux relations suivantes a lieu :

$$
\text { (i) } q_{m}=q /(q, m) \text {; } \quad \text { (ii) } q_{m} \geqslant Q /(2 m) \text {. }
$$

Démonstration. Il existe des entiers $a$ et $a_{m}$, avec $(a, q)=\left(a_{m}, q_{m}\right)=1$, tels que $|\vartheta-a / q| \leqslant 1 /(q Q),\left|m \vartheta-a_{m} / q_{m}\right| \leqslant 1 /\left(2 q_{m} Q\right)$, et donc

$$
\left|\frac{a}{q}-\frac{a_{m}}{m q_{m}}\right| \leqslant \frac{1}{Q}\left\{\frac{1}{q}+\frac{1}{2 m q_{m}}\right\}
$$

Si $a / q=a_{m} /\left(m q_{m}\right)$, alors $a_{m} / q_{m}=\{a m /(m, q)\} /\{q /(m, q)\}$ et, puisque les deux membres de cette dernière égalité sont des fractions irréductibles, $q_{m}=q /(m, q)$. Sinon,

$$
\frac{1}{q m q_{m}} \leqslant \frac{1}{Q}\left\{\frac{1}{q}+\frac{1}{2 m q_{m}}\right\}
$$

d'où $\frac{1}{2} Q \leqslant Q-\frac{1}{2} q \leqslant m q_{m}$ et donc $q_{m} \geqslant Q /(2 m)$.

Nous aurons également l'usage du classique résultat d'approximation suivant, dont nous rappelons la démonstration pour la commodité du lecteur. 
Lemme 6.3. Soient $\vartheta \in \mathbb{R}, a \in \mathbb{Z}, q \geqslant 1,(a, q)=1, \vartheta=a / q+\beta,|\beta| \leqslant 1 / q^{2}$, $t \in \mathbb{N}, 0 \leqslant v \leqslant q-1$. Il y a au plus six valeurs de $r \in \mathbb{N}, 0 \leqslant r<q$ telles que l'on ait

$$
\|\vartheta(t q+r)\| \in] v / q,(v+1) / q]
$$

Démonstration. Pour chaque $t$, nous avons $\|\vartheta(t q+r)\|=\left\|\alpha_{r}\right\|$ avec $\alpha_{r}:=$ $r a / q+r \beta+t q \beta$. Lorsque $0 \leqslant r \neq s<q$, et si $\left\langle\alpha_{r}\right\rangle-\frac{1}{2}$ et $\left\langle\alpha_{s}\right\rangle-\frac{1}{2}$ ont le même signe, nous pouvons écrire $\left|\left\|\alpha_{s}\right\|-\left\|\alpha_{r}\right\|\right|=\left\|\alpha_{s}-\alpha_{r}\right\|>\|(s-r) a / q\|-1 / q$. Il y a donc au plus six valeurs de $r, 0 \leqslant r<q$, telles que $\alpha_{r}$ appartienne à l'intervalle ]$v / q,(v+1) / q$ ] modulo 1 .

Nous rappelons enfin certaines propriétés connues du développement en fraction continue des nombres irrationnels. Rappelons la définition $(2 \cdot 1)$. Pour chaque $\vartheta \in \mathbb{R} \backslash \mathbb{Q}$, l'ensemble des entiers de la forme $q(\vartheta ; Q)(Q \geqslant 1)$ coïncide avec celui des dénominateurs des réduites de $\vartheta$ — cf., par exemple, [19], théorèmes 16 et 17. Notant $\left\{q_{m}\right\}_{m=1}^{\infty}=\left\{q_{m}(\vartheta)\right\}_{m=1}^{\infty}$ la suite des dénominateurs des réduites de $\vartheta$, on a $q_{0}=1$ et

$$
q_{m}=\min \left\{q:\|q \vartheta\|<\left\|q_{m-1} \vartheta\right\|\right\} \quad(m \geqslant 1) .
$$

La croissance de la suite des $q_{m}$ est au moins exponentielle. On a par exemple $q_{m+2} \geqslant q_{m+1}+q_{m} \geqslant 2 q_{m}$.

Désignant par $a_{m} / q_{m}$ la $m$-ième réduite de $\vartheta \in \mathbb{R} \backslash \mathbb{Q}$, nous posons

$$
\varepsilon_{m}:=\vartheta-a_{m} / q_{m}
$$

La quantité $(-1)^{m} \varepsilon_{m}$ est de signe constant. On a

$$
\frac{1}{q_{m}\left(q_{m+1}+q_{m}\right)}<\left|\varepsilon_{m}\right|<\frac{1}{q_{m} q_{m+1}} \quad(m \geqslant 1)
$$

d'où

$$
\left|\varepsilon_{m}\right| \asymp \frac{1}{q_{m} q_{m+1}} \quad(m \geqslant 1)
$$

Soient $B$ une constante positive et $Q_{x}:=x /(\log x)^{B}(x \geqslant 2)$. L'ensemble des nombres réels $x$ tels que $q\left(\vartheta ; Q_{x}\right)=q_{m}$ est l'intervalle défini par les conditions $q_{m} \leqslant Q_{x}<q_{m+1} ;$ nous le notons $\left[\xi_{m}, \xi_{m+1}[\right.$. Nous utiliserons les relations

$$
\xi_{m} \asymp q_{m}\left(\log q_{m}\right)^{B}, \quad\left|\varepsilon_{m}\right| \xi_{m} \asymp \frac{\left(\log q_{m}\right)^{B}}{q_{m+1}}, \quad\left|\varepsilon_{m}\right| \xi_{m+1} \asymp \frac{\left(\log q_{m+1}\right)^{B}}{q_{m}} .
$$




\subsection{Estimations de $E(x, y ; \vartheta)$ et $Z(x, y ; \vartheta)$}

Dans la suite de ce travail, nous utilisons systématiquement les notations

$$
Y:=\mathrm{e}^{\sqrt{\log y}}, \quad Y_{1}:=y^{\log _{2} y} .
$$

Lemme 6.4. Il existe des constantes positives $c_{1}$ et $c_{2}$ telles que, pour tous $x \geqslant 2$, $y \geqslant 2, \vartheta \in \mathbb{R}$ vérifiant $\|\vartheta\| x \leqslant Y^{c_{1}}$, on ait

$$
Z(x, y ; \vartheta)=\varrho(u) Z(x, x ; \vartheta)+O\left(R_{1}(x, y ; \vartheta)\right)
$$

avec

$$
R_{1}(x, y ; \vartheta):=\Psi(x, y) \frac{\log (u+1)}{\log y} \frac{\log \left(1+x^{2}\|\vartheta\|^{2}\right)}{x\|\vartheta\|}+\frac{x}{Y^{c_{2}}} .
$$

Démonstration. Nous pouvons nous restreindre au cas $x \geqslant y$ car le résultat est trivialement vérifié dans la circonstance opposée. Nous supposons également, quitte à changer $\vartheta$ en $-\vartheta$, que $\vartheta=\|\vartheta\|$.

Il est établi au §2 de [4] que l'on a, sous les conditions de l'énoncé,

$$
E(x, y ; \vartheta)=\varrho(u) E(x, x ; \vartheta)+O\left(\Psi(x, y) \frac{\log (u+1)}{\log y} \frac{\log (1+x\|\vartheta\|)}{x\|\vartheta\|}+\frac{x}{Y^{c_{2}}}\right) .
$$

Cela implique bien (6.6) si $\|\vartheta\|>1 / x$. Il nous suffit donc d'établir l'estimation

$$
Z(x, y ; \vartheta)-\varrho(u) Z(x, x ; \vartheta) \ll \Psi(x, y) \frac{\log (u+1)}{\log y} x\|\vartheta\|+\frac{x}{Y^{c_{2}}}
$$

lorsque $\|\vartheta\| \leqslant 1 / x$.

Le membre de gauche de (6.7) est trivialement

$$
\ll \Psi(x, y)+x \varrho(u) \ll x u^{-u}+x^{1 / 4},
$$

où la dernière estimation résulte de $(6 \cdot 2)$. Cette majoration étant $\ll x / Y$ lorsque $\log x>(\log y)^{3 / 2}$, nous pouvons supposer dans la suite que

$$
y \leqslant x \leqslant \exp \left\{(\log y)^{3 / 2}\right\} .
$$

D'après de Bruijn [5], on a dans ce domaine

$$
\Psi(x, y)=\Lambda(x, y)+O\left(\Psi(x, y) / Y^{2 c_{2}}\right)
$$

avec

$$
\Lambda(x, y):= \begin{cases}x \int_{0-}^{\infty} \varrho(u-v) \mathrm{d}\left(\left[y^{v}\right] / y^{v}\right) & (x \notin \mathbb{Z}), \\ \Lambda(x+, y) & (x \in \mathbb{Z}) .\end{cases}
$$


Il vient

$$
\begin{aligned}
Z(x, y ; \vartheta) & -\varrho(u) Z(x, x ; \vartheta) \\
& =\int_{x / Y^{c_{2}}}^{x} \sin (2 \pi \vartheta t) \mathrm{d}\{\Lambda(t, y)-[t] \varrho(u)\}+O\left(x / Y^{c_{2}}\right)
\end{aligned}
$$

Il est nécessaire d'évaluer la mesure figurant au membre de droite. On a, pour y fixé, l'égalité entre mesures en $t$ :

$$
\mathrm{d} \Lambda(t, y)=\lambda(t, y) \mathrm{d} t+t \mathrm{~d}([t] / t)
$$

avec

$$
\lambda(t, y):=\frac{\Lambda(t, y)}{t}+\frac{1}{\log y} \int_{0-}^{\infty} \varrho^{\prime}\left(u_{t, y}-v\right) \mathrm{d}\left(\left[y^{v}\right] / y^{v}\right),
$$

où l'on a employé la notation $(2 \cdot 5)$. On peut aisément établir l'estimation

$$
\lambda(t, y)=\varrho\left(u_{t, y}\right)+O\left(\frac{\varrho\left(u_{t, y}\right) \log \left(u_{t, y}+1\right)}{\log y}\right) \quad\left(y \geqslant(\log t)^{2}\right) .
$$

Nous omettons les détails qui sont semblables à ceux de la preuve du théorème 3 de [14]. Compte tenu de la majoration

$$
\varrho(u-v) \ll \varrho(u)\{u \log (1+u)\}^{v} \quad(0 \leqslant v \leqslant 1)
$$

qui résulte du corollaire III.5.8.4 de [29], cela implique

$$
\lambda(t, y)=\varrho\left(u_{t, y}\right)+O\left(\frac{\varrho(u) \log (u+1)}{\log y}\right) \quad\left(x / Y^{c_{2}} \leqslant t \leqslant x\right)
$$

dans le domaine $(6 \cdot 8)$.

Il découle de $(6 \cdot 10)$ que

$$
\mathrm{d}\{\Lambda(t, y)-[t] \varrho(u)\}=\lambda_{1}(t, y) \mathrm{d} t+\{\varrho(u)-1\} \mathrm{d}\langle t\rangle
$$

avec

$$
\lambda_{1}(t, y):=\lambda(t, y)-\varrho(u)+\langle t\rangle / t .
$$

Cette dernière quantité peut être évaluée grâce à (6•11). On a, dans le domaine (6•8),

$$
\lambda_{1}(t, y) \ll \frac{\varrho(u) \log (u+1)\{\log (x / t)+1\}}{\log y} \quad\left(x / Y^{c_{2}} \leqslant t \leqslant x\right) .
$$

Reportons (6.12) dans (6.9) en tenant compte de (6.13). La contribution de la mesure $\mathrm{d}\langle t\rangle$ est nulle. Estimons celle de la mesure absolument continue en employant la borne triviale $|\sin (2 \pi \vartheta t)| \ll\|\vartheta\| x$. Nous obtenons bien l'estimation requise $(6 \cdot 7)$.

Le résultat suivant, qui fait l'objet du corollaire 3 de [2], nous permet de traiter le cas des grandes valeurs de $q(\vartheta ; Q)$. 
Lemme 6.5. On a uniformément pour $x \geqslant y \geqslant 2, R \geqslant 1, \vartheta \in \mathbb{R}, q(\vartheta ; x / R) \geqslant R$,

$$
\begin{gathered}
|E(x, y ; \vartheta)| \ll x(\log x)^{4}\left\{R^{-1 / 4}+\mathrm{e}^{-\sqrt{\log x}}\right\}, \\
\left|E_{\mu}(x, y ; \vartheta)\right| \ll x(\log x)^{4}\left\{R^{-1 / 4}+\mathrm{e}^{-\sqrt{\log x}}\right\} .
\end{gathered}
$$

Nous traitons finalement le cas des petites valeurs de $q(\vartheta ; Q)$. Nous introduisons les notations

$$
\begin{aligned}
\alpha(q) & :=5 /(6+\log q), \\
J_{q}(x, y) & :=\frac{\log q}{\log y}(\log (u+1))^{2}+\left(\frac{y}{x}\right)^{\alpha(q)}, \\
R_{q}(x, y) & :=\Psi(x, y) \frac{2^{\omega(q)} \log q}{\varphi(q) \log y} J_{q}(x, y) .
\end{aligned}
$$

Lemme 6.6. Soit $A>0$. On a uniformément pour $\vartheta \in \mathbb{R}, x \geqslant y \geqslant 2$, $Q_{x}:=x /(\log x)^{4 A+16}, q:=q\left(\vartheta ; Q_{x}\right) \geqslant 2, a \in \mathbb{Z},(a, q)=1,|q \vartheta-a| \leqslant 1 / Q_{x}$, $\vartheta_{q}:=\vartheta-a / q$,

$$
Z(x, y ; \vartheta)=-x \frac{\Lambda(q)}{\varphi(q)} \frac{\varrho^{\prime}(u)}{\log y} \frac{\left\{\sin \left(\pi \vartheta_{q} x\right)\right\}^{2}}{\pi \vartheta_{q} x}+O\left(R_{q}(x, y)+\frac{x}{(\log x)^{A}}\right) .
$$

Démonstration. L'estimation $(6 \cdot 14)$ peut être établie par des calculs très voisins de ceux qui sont menés dans les preuves des théorèmes 1 et 2 de [2], aussi nous nous bornons à indiquer les étapes essentielles de la déduction.

Posons

$$
S_{q}(t):=\frac{1}{t} \sum_{n \leqslant t} \frac{\mu(q /(n, q))}{\varphi(q /(n, q))}, \quad V_{q}(x, y):= \begin{cases}x \int_{-\infty}^{\infty} \varrho(u-v) \mathrm{d} S_{q}\left(y^{v}\right) & \left(x \notin \mathbb{N}^{*}\right) \\ V_{q}(x+, y) & \left(x \in \mathbb{N}^{*}\right) .\end{cases}
$$

Le point (ii) du théorème 1 de [2] fournit une évaluation de $V_{q}(x, y)$ lorsque $u$ n'est pas trop proche d'un entier par excès. La démonstration fournit en fait ${ }^{(14)}$ le résultat suivant, que par souci de concision, nous énonçons seulement dans le cas $k=1$ de la forme originale : il existe des constantes positives $\kappa_{0}, \kappa_{1}$ telles que, pour $x \geqslant 3, \exp \left\{\kappa_{0}\left(\log _{2} x\right)^{2}\right\} \leqslant y \leqslant x, 2 \leqslant q \leqslant Y^{\kappa_{1}}$, on ait

$$
V_{q}(x, y)=-x \frac{\Lambda(q) \varrho^{\prime}(u)}{\varphi(q) \log y}+O\left(R_{q}(x, y)\right) .
$$

Nous sommes maintenant en position de décrire sommairement la preuve de $(6 \cdot 14)$.

14. Voir les calculs de la p. 59 de [2]. 
Nous observons d'abord que la majoration (6.2) nous permet, compte tenu de la présence du terme $x /(\log x)^{A}$ dans le terme résiduel de (6.14), de limiter l'étude au domaine

$$
2 \leqslant y \leqslant x \leqslant Y_{1}:=y^{\log _{2} y} .
$$

Lorsque cette condition n'est pas réalisée, (6-14) est impliquée par l'estimation triviale $Z(x, y ; \vartheta) \ll \Psi(x, y)$. Au vu du Lemme 6.5 , nous pouvons également supposer que

$$
2 \leqslant q \leqslant(\log x)^{4 A+16} .
$$

Si $\vartheta_{q}=0$, le théorème 1 de [2] implique

$$
E(x, y ; a / q)=V_{q}(x, y)+O\left(\frac{x}{(\log x)^{A}}\right),
$$

d'où, puisque $V_{q}(x, y)$ est réel,

$$
Z(x, y ; a / q)=\Im m E(x, y ; a / q) \ll x /(\log x)^{A} .
$$

Nous nous plaçons dorénavant dans l'hypothèse $\vartheta_{q} \neq 0$. Posons

$$
x_{1}:=\max \left(x /(\log x)^{A}, y\right) .
$$

Alors on a sous les conditions $(6 \cdot 16)$ et $(6 \cdot 17)$

$$
E\left(x_{1}, y ; \vartheta\right) \ll x /(\log x)^{A} .
$$

En effet, cette estimation est triviale si $y \leqslant x /(\log x)^{A}$ et, dans le cas contraire, elle résulte immédiatement de la majoration

$$
E(y, y ; \vartheta) \ll 1 /\|\vartheta\| \leqslant 2 q \ll(\log x)^{4 A+16} .
$$

Nous déduisons de $(6 \cdot 18)$ que

$$
E(x, y ; \vartheta)=\int_{x_{1}}^{x} \mathrm{e}\left(\vartheta_{q} t\right) \mathrm{d} E(t, y ; a / q)+O\left(x /(\log x)^{A}\right) .
$$

Posons

$$
v_{q}(t, y):=V_{q}(t, y) / t
$$

Avec des calculs essentiellement identiques à ceux du paragraphe 2.5 de [2] et reposant sur l'évaluation de $E(t, y ; \vartheta)$ obtenue au théorème 1 de ce même travail, nous pouvons déduire de (6.19) que l'on a

$$
E(x, y ; \vartheta)=\int_{x_{1}}^{x} \mathrm{e}\left(\vartheta_{q} t\right) v_{q}(t, y) \mathrm{d} t+O\left(R_{q}(x, y)+x /(\log x)^{A}\right) .
$$


La fonction $v_{q}(t, y)$ est réelle. En égalant les parties imaginaires des deux membres, nous obtenons

$$
\begin{aligned}
Z(x, y ; \vartheta) & =\int_{x_{1}}^{x} \sin \left(2 \pi \vartheta_{q} t\right) v_{q}(t, y) \mathrm{d} t+O\left(R_{q}(x, y)+x /(\log x)^{A}\right) \\
& =\frac{-\Lambda(q)}{\varphi(q) \log y} \int_{x_{1}}^{x} \sin \left(2 \pi \vartheta_{q} t\right) \varrho^{\prime}\left(u_{t, y}\right) \mathrm{d} t+O\left(R_{q}(x, y)+x /(\log x)^{A}\right),
\end{aligned}
$$

où le terme d'erreur issu de celui de (6-15) a été traité en majorant trivialement $\left|\sin \left(2 \pi \vartheta_{q} t\right)\right|$ par 1 et en utilisant l'estimation du lemme 4.1 de [14] :

$$
\int_{0}^{\infty} \varrho(u-v) y^{-v} \mathrm{~d} v \ll \varrho(u) / \log y,
$$

valable pour $y \geqslant 2,0 \leqslant u \leqslant \sqrt{y}$. Une intégration par parties permet maintenant d'écrire

$$
\begin{aligned}
\int_{x_{1}}^{x} \sin \left(2 \pi \vartheta_{q} t\right) \varrho^{\prime}\left(u_{t, y}\right) \mathrm{d} t-\frac{x \varrho^{\prime}(u)\left\{1-\cos \left(2 \pi \vartheta_{q} x\right\}\right.}{2 \pi \vartheta_{q} x} \\
=\int_{x_{1}}^{x} \frac{\left\{\cos \left(2 \pi \vartheta_{q} t\right)-1\right\} \varrho^{\prime \prime}\left(u_{t, y}\right)}{2 \pi \vartheta_{q} t \log y} \mathrm{~d} t+O\left(\frac{x}{(\log x)^{A}}\right) \\
\ll \frac{x \varrho(u)\{\log (u+1)\}^{2}}{\log y}+\frac{x}{(\log x)^{A}} .
\end{aligned}
$$

où l'on a utilisé les majorations

$$
\begin{aligned}
& \varrho^{\prime \prime}\left(u_{t, y}\right) \ll \varrho(u)\{\log (u+1)\}^{2} \quad\left(x /(\log x)^{A} \leqslant t \leqslant x\right), \\
& \left\{1-\cos \left(2 \pi \vartheta_{q} t\right)\right\} / 2 \pi \vartheta_{q} \ll t \quad(t \in \mathbb{R}) .
\end{aligned}
$$

Cela implique bien l'estimation requise (6-14).

\section{3. Estimations de $E_{\tau_{z}}(x, y ; \vartheta)$ et $Z_{\tau_{z}}(x, y ; \vartheta)$}

Nous conservons les notations du paragraphe 6.1. La fonction $\tau_{z}$ est définie à la fin du paragraphe 4 . Nous utiliserons à plusieurs reprises dans la suite les inégalités

$$
\left|\tau_{z}(m)\right| \leqslant \tau_{\kappa}(m) \leqslant \tau_{\lambda}(m) \quad(|z| \leqslant \kappa \leqslant \lambda, m \geqslant 1) .
$$

Les deux lemmes suivants fournissent des majorations de $E_{\tau_{z}}(x, y ; \vartheta)$ en fonction des approximations rationnelles de $\vartheta$.

Lemme 6.7. Soit $\kappa \geqslant 1$. Sous les conditions $z \in \mathbb{C},|z| \leqslant \kappa, x \geqslant 2, q \in \mathbb{N}$, $1 \leqslant q \leqslant x, a \in \mathbb{Z},(a, q)=1,|\vartheta-a / q| \leqslant 1 / q^{2}$, on a

$$
E_{\tau_{z}}(x, x ; \vartheta) \ll_{\kappa} x(\log x)^{3+\kappa^{2} / 2}\left\{\frac{\mathrm{e}^{\sqrt{\log q}}}{\sqrt{q}}+\left(\frac{q}{x}\right)^{1 / 4}+\mathrm{e}^{-\sqrt{\log x}}\right\} .
$$


Remarque. Nous n'avons pas cherché à optimiser la puissance de $\log x$ dans $(6 \cdot 21)$. Démonstration. Nous utilisons la méthode de [8], qui correspond au cas de la fonction de Möbius, c'est-à-dire $z=-1$. Posons $y:=\min \left\{\mathrm{e}^{2 \sqrt{\log x}}, \sqrt{x / q}\right\}$. La contribution à $E_{\tau_{z}}(x, x ; \vartheta)$ des entiers $n$ tels que $P(n) \leqslant y$ est

$$
\begin{aligned}
& \ll \sum_{n \in S(x, y)} \tau_{\kappa}(n) \leqslant\left\{\Psi(x, y) \sum_{n \leqslant x} \tau_{\kappa}(n)^{2}\right\}^{1 / 2} \\
& \ll x u^{-u / 2}(\log x)^{\left(\kappa^{2}-1\right) / 2} \ll x \mathrm{e}^{-2 \sqrt{\log x}}
\end{aligned}
$$

où l'on a fait appel à $(6 \cdot 2) .{ }^{(15)}$ Décomposons les entiers $n$ restants sous la forme $n=m p$ avec $p=P(n)>y$. Nous obtenons

$$
\begin{aligned}
E_{\tau_{z}}(x, x ; \vartheta) & =\sum_{\substack{m p \leqslant x \\
p \geqslant P(m), p>y}} \tau_{z}(m p) \mathrm{e}(\vartheta m p)+O\left(x \mathrm{e}^{-2 \sqrt{\log x}}\right) \\
& =T_{1}+T_{2}+T_{3}+O\left(x \mathrm{e}^{-2 \sqrt{\log x}}\right),
\end{aligned}
$$

où $T_{1}, T_{2}, T_{3}$ correspondent respectivement aux conditions supplémentaires $m \leqslant y$, $m>y$ et $p \nmid m, m>y$ et $p \mid m$.

Nous avons

$$
\begin{aligned}
T_{1} & =z \sum_{m \leqslant y} \tau_{z}(m) \sum_{y<p \leqslant x / m} \mathrm{e}(\vartheta m p) \\
& \ll(\log x)^{3} \sum_{m \leqslant y} \tau_{\kappa}(m)\left\{\frac{x}{m \sqrt{q_{m}}}+\left(\frac{x}{m}\right)^{4 / 5}+\sqrt{\frac{q_{m} x}{m}}\right\}
\end{aligned}
$$

où l'on a posé $q_{m}:=q /(q, m)$ et où la somme en $p$ a été estimée selon la majoration de Vaughan — cf., par exemple, Davenport [9], chapitre 25. La fonction $\tau_{\kappa}$ est sousmultiplicative lorsque $\kappa \geqslant 1$. On peut donc écrire

$$
\begin{aligned}
\sum_{m \leqslant y} \frac{\tau_{\kappa}(m) \sqrt{(m, q)}}{m} & \leqslant \sum_{d \mid q} \frac{\tau_{\kappa}(d)}{\sqrt{d}} \sum_{n \leqslant y / d} \frac{\tau_{\kappa}(n)}{n} \\
& \ll(\log y)^{\kappa} \prod_{p \mid q}\left(1+\frac{\kappa}{\sqrt{p}}+\frac{\kappa(\kappa+1)}{2 p}\right) \\
& \ll(\log x)^{\kappa / 2} \mathrm{e}^{\sqrt{\log q}} .
\end{aligned}
$$

Par ailleurs, on déduit de l'estimation classique de la fonction sommatoire de $\tau_{\kappa}(n)$ (cf., par exemple, [29], chap. II.5) que l'on a

$$
\sum_{m \leqslant y} \frac{\tau_{\kappa}(m)}{m^{1-\sigma}} \ll_{\sigma} y^{\sigma}(\log y)^{\kappa-1} \quad(0<\sigma \leqslant 1) .
$$

15. Voir Smida [25], [26], pour des estimations précises de $\sum_{n \in S(x, y)} \tau_{\kappa}(n)$. 
En reportant ces majorations dans $(6 \cdot 23)$, nous obtenons

$$
T_{1} \ll_{\kappa} x(\log x)^{3+\kappa / 2}\left\{\frac{\mathrm{e}^{\sqrt{\log q}}}{\sqrt{q}}+\left(\frac{q}{x}\right)^{1 / 4}+\mathrm{e}^{-\sqrt{\log x}}\right\} .
$$

Pour majorer $T_{2}$, nous utilisons une technique classique due à Vinogradov. En intervertissant les sommations et en scindant la sommation en $p$ en intervalles dyadiques, nous obtenons

$$
T_{2} \ll \sum_{0 \leqslant j<J} W\left(2^{j} y\right)
$$

avec $J:=\frac{\log (x / y)}{\log y}$ et

$$
\begin{aligned}
W(P) & :=\sum_{P<p \leqslant \min (2 P, x / y)}\left|\sum_{\substack{y<m \leqslant x / p \\
P(m)<p}} \tau_{z}(m) e(\vartheta m p)\right| \\
& \leqslant \sum_{P<k \leqslant \min (2 P, x / y)}\left|\sum_{\substack{y<m \leqslant x / k \\
P(m)<k}} \tau_{z}(m) e(\vartheta m k)\right|,
\end{aligned}
$$

où nous avons étendu la somme en $p$ à tous les entiers $k$ du même intervalle. Pour chaque $P, y \leqslant P \leqslant x / y$, l'inégalité de Cauchy-Schwarz fournit

$$
\begin{aligned}
W(P)^{2} & \leqslant P \sum_{P<k \leqslant 2 P} \sum_{\substack{y<m, n \leqslant x / k \\
P(m n)<k}} \tau_{z}(m n) e(k \vartheta(n-m)) \\
& =P \sum_{y<m, n \leqslant x / P} \tau_{z}(m n) \sum_{\max (P, P(m n))<k \leqslant \min (2 P, x / m, x / n)} e(k \vartheta(n-m)) \\
& \ll P \sum_{1 \leqslant m, n \leqslant x / P} \tau_{\kappa}(m n) \min (P, 1 /\|\vartheta(n-m)\|) \\
& \ll P \sum_{0 \leqslant h \leqslant x / P} \min (P, 1 /\|\vartheta h\|) \sum_{h+1 \leqslant m \leqslant x / P} \tau_{\kappa}(m(m-h)) .
\end{aligned}
$$

Une seconde application de l'inégalité de Cauchy-Schwarz nous permet d'écrire

$$
\sum_{h+1 \leqslant m \leqslant x / P} \tau_{\kappa}(m(m-h)) \ll \sum_{1 \leqslant m \leqslant x / P} \tau_{\kappa}(m)^{2} \ll \frac{x}{P}(\log x)^{\kappa^{2}-1} .
$$

Pour estimer la somme en $h$ de $(6 \cdot 27)$, nous écrivons $\vartheta=a / q+\beta$ avec $|\beta| \leqslant 1 / q^{2}$ et $h=t q+r$ avec $0 \leqslant r<q$. Le Lemme 6.3 implique

$$
\begin{aligned}
\sum_{0 \leqslant h \leqslant x / P} \min (P, 1 /\|\vartheta h\|) & \ll \sum_{0 \leqslant t \leqslant x / P q} \sum_{0 \leqslant r<q} \min (P, 1 /\|a r / q\|) \\
& \ll(1+x / P q)(P+q \log q) \\
& \ll x \log x\left(\frac{P}{x}+\frac{q}{x}+\frac{1}{q}+\frac{1}{P}\right) \ll x \log x\left(\frac{1}{y}+\frac{q}{x}+\frac{1}{q}\right) .
\end{aligned}
$$


En reportant cette estimation et $(6 \cdot 28)$ dans $(6 \cdot 27)$, nous obtenons

$$
W(P) \ll x(\log x)^{\kappa^{2} / 2}\left(\frac{1}{y}+\frac{q}{x}+\frac{1}{q}\right)^{1 / 2} \quad(y \leqslant P \leqslant x / y),
$$

ce qui fournit, par insertion dans (6·25), la majoration

$$
T_{2} \ll x(\log x)^{1+\kappa^{2} / 2}\left\{\frac{1}{\sqrt{q}}+\left(\frac{q}{x}\right)^{1 / 4}+\mathrm{e}^{-\sqrt{\log x}}\right\} .
$$

Pour évaluer $T_{3}$, nous nous contentons d'une majoration triviale de l'exponentielle :

$$
T_{3} \ll \sum_{p>y} \sum_{m \leqslant x / p^{2}} \tau_{\kappa}(m) \ll x(\log x)^{\kappa-1} \sum_{p>y} 1 / p^{2} \ll \frac{x(\log x)^{\kappa-1}}{y} .
$$

En regroupant $(6 \cdot 24),(6 \cdot 30)$ et $(6 \cdot 31)$, nous obtenons bien $(6 \cdot 21)$.

Lemme 6.8. Soit $\kappa \geqslant 1$. On a uniformément pour $z \in \mathbb{C},|z| \leqslant \kappa, x \geqslant y \geqslant 2$, $R \geqslant 1, \vartheta \in \mathbb{R}, q(\vartheta ; x / R) \geqslant R$,

$$
E_{\tau_{z}}(x, y ; \vartheta) \ll x(\log x)^{\kappa^{2}+3}\left\{R^{-1 / 4}+\mathrm{e}^{-\sqrt{\log x}}\right\} .
$$

Démonstration. Nous pouvons manifestement supposer $R \leqslant \mathrm{e}^{4 \sqrt{\log x}}$ et, au vu de $(6 \cdot 2)$ et $(6 \cdot 22), y>R^{2}$. La proposition 1 de [2] appliquée avec $f=\tau_{z}$, $A^{2}=(\log x)^{\kappa^{2}-1}$, implique sous les hypothèses effectuées

$$
E_{\tau_{z}}(x, y ; \vartheta) \ll x(\log x)^{\kappa^{2}+1 / 2}\left\{\sqrt{\frac{y}{x}}+\frac{1}{\sqrt{R}}+\mathrm{e}^{-\sqrt{\log x}}\right\} .
$$

Par ailleurs, la proposition 2 de [2], appliquée avec $f=\tau_{z}, \alpha_{1}=\kappa, \alpha_{2}=0$, fournit, sous l'hypothèse supplémentaire $y>x / \sqrt{R}$,

$$
E_{\tau_{z}}(x, y ; \vartheta) \ll\left|E_{\tau_{z}}(x, x ; \vartheta)\right|+x(\log x)^{\kappa+3} R^{-1 / 4} .
$$

La relation (6.33) implique l'estimation annoncée (6.32) si $y \leqslant x / \sqrt{R}$. Dans le cas contraire, nous reportons la majoration $(6 \cdot 21)$ pour $\left|E_{\tau_{z}}(x, x ; \vartheta)\right|$ dans $(6 \cdot 34)$; cela fournit encore $(6 \cdot 32)$. 
Lemme 6.9. Soit $\kappa \geqslant 1$. On a uniformément pour $\vartheta \in \mathbb{R}, z \in \mathbb{C},|z| \leqslant \kappa, y \geqslant 2$, $R \geqslant 1$ et $q(\vartheta ; y / R) \geqslant R$,

$$
\sum_{\substack{P(n) \leqslant y \\ n>y}} \frac{\tau_{z}(n)}{n} \mathrm{e}(\vartheta n) \ll \frac{(\log y)^{\kappa^{2}+5}}{R^{1 / 4}}+\mathrm{e}^{-\frac{1}{2} \sqrt{\log y}} .
$$

En particulier, si $q\left(\vartheta ; y /(\log y)^{4 \kappa^{2}+21}\right) \geqslant(\log y)^{4 \kappa^{2}+21}$, on a

$$
U_{\tau_{z}}(\vartheta ; y)=o(1) \quad(y \rightarrow \infty) .
$$

Démonstration. Il suffit d'intégrer par parties l'estimation (6.32) en utilisant la majoration triviale issue de (6.22) pour $x>Y_{1}$. Nous omettons les détails.

Lorsque $\vartheta$ est un nombre rationnel à «petit » dénominateur, nous pouvons donner un développement asymptotique pour $E_{\tau_{z}}(x, x ; \vartheta)$.

Pour $q \in \mathbb{N}^{*}, z \in \mathbb{C}, s=\sigma+i \tau, \sigma \geqslant 1-c /\left(1+\log ^{+}|\tau|\right)$, avec $c$ assez petite, nous posons

$$
G_{q}(s, z):=\frac{\{\zeta(s)(s-1)\}^{z}}{q^{s-1}} \prod_{p^{\nu} \| q}\left(1-p^{-s}\right)^{z}\left\{\sum_{\ell \geqslant 0} \frac{\tau_{z}\left(p^{\ell+\nu}\right)}{p^{\ell s}}-\frac{\tau_{z}\left(p^{\nu-1}\right) p^{s-1}}{1-1 / p}\right\}
$$

où le logarithme complexe est pris en détermination principale.

Lemme 6.10. Soient $A>0, \kappa \geqslant 1$ et $J \in \mathbb{N}$. On a, uniformément pour $x \geqslant 2$, $q \in \mathbb{N}, 1 \leqslant q \leqslant(\log x)^{A}, a \in \mathbb{Z},(a, q)=1, z \in \mathbb{C},|z| \leqslant \kappa$,

$$
E_{\tau_{z}}(x, x ; a / q)=\frac{x}{q}(\log x)^{z-1}\left\{\sum_{j=0}^{J} \frac{\lambda_{j}(z, q)}{(\log x)^{j}}+O\left(\frac{\tau_{\kappa+1}(q)(\log 2 q)^{J+2}}{(\log x)^{J+1}}\right)\right\}
$$

avec

$$
\lambda_{j}(z, q):=\frac{1}{\Gamma(z-j)} \frac{\partial^{j} G_{q}}{\partial s^{j}}(1, z) \ll_{j, \kappa} \tau_{\kappa+1}(q)(\log 2 q)^{j+1} \quad(0 \leqslant j \leqslant J) .
$$

Démonstration. Une variante de ces résultats est établie au lemme 4 de [12] pour la fonction $n \mapsto z^{\Omega(n)}$ à la place de $n \mapsto \tau_{z}(n)$. La technique employée dans [12] est encore valable ici, moyennant quelques modifications contingentes que nous nous contentons de décrire brièvement.

Posons

$$
F(s, z, a / q):=\sum_{n=1}^{\infty} \tau_{z}(n) \frac{e(a n / q)}{n^{s}} \quad(\sigma>1) .
$$

En classant les entiers $n$ selon leurs résidus modulo $q$ et en introduisant les caractères de Dirichlet pour repérer les entiers en progressions arithmétiques, on obtient l'identité

$$
F(s, z, a / q)=F_{1}(s, z, q)+F_{2}(s, z, a / q)
$$


avec

$$
\left\{\begin{aligned}
F_{1}(s, z, q) & :=\sum_{m \mid q} \frac{\mu(m)}{\varphi(m)} \frac{m^{s}}{q^{s}} \sum_{n \geqslant 1} \frac{\tau_{z}(n q / m) \chi_{0, m}(n)}{n^{s}} \\
F_{2}(s, z, a / q) & :=\sum_{m \mid q} \frac{m^{s}}{\varphi(m) q^{s}} \sum_{\substack{\chi \neq \chi_{0, m} \\
\chi \bmod m}} G_{m}(\bar{\chi}) \chi(a) \sum_{n \geqslant 1} \frac{\tau_{z}(n q / m) \chi(n)}{n^{s}}
\end{aligned}\right.
$$

où $\chi_{0, m}$ est le caractère principal modulo $m$ et $G_{m}(\chi)$ désigne génériquement la somme de Gauss associée à un caractère $\chi$ de module $m$.

On a

$$
F_{1}(s, z, q)=\sum_{m \mid q} \frac{\mu(m)}{\varphi(m)} T_{m}(q / m, s, z)
$$

avec, pour $m \mid q, \mu(m) \neq 0$, et en posant $r:=q / m$,

$$
\begin{aligned}
T_{m}(r, s, z) & :=\sum_{n \geqslant 1} \frac{\tau_{z}(r n) \chi_{0, m}(n)}{(r n)^{s}}=L\left(s, \chi_{0, q}\right)^{z} \prod_{p^{a} \| r} \sum_{b \geqslant 0} \frac{\tau_{z}\left(p^{a+b}\right) \chi_{0, m}\left(p^{b}\right)}{p^{(a+b) s}} \\
& =\zeta(s)^{z} \prod_{p \mid q}\left(1-p^{-s}\right)^{z} \prod_{p^{\nu} \| q, p \nmid m} \sum_{b \geqslant \nu} \frac{\tau_{z}\left(p^{b}\right)}{p^{b s}} \prod_{p^{\nu} \| q, p \mid m} \frac{\tau_{z}\left(p^{\nu-1}\right)}{p^{(\nu-1) s}} \\
& =\zeta(s)^{z} \prod_{p \mid q}\left(1-p^{-s}\right)^{z} M(q ; s) M_{q}(m ; s),
\end{aligned}
$$

où $q \mapsto M(q ; s)$ et $m \mapsto M_{q}(m ; s)$ sont des fonctions multiplicatives satisfaisant à

$$
\begin{aligned}
& M\left(p^{\nu} ; s\right):=\sum_{\ell \geqslant \nu} \tau_{z}\left(p^{\ell}\right) p^{-\ell s}, \\
& M_{q}(p ; s):=\tau_{z}\left(p^{\nu-1}\right) p^{-(\nu-1) s} / M\left(p^{\nu} ; s\right) \quad\left(p^{\nu} \| q\right) .
\end{aligned}
$$

En reportant dans $(6 \cdot 39)$, il suit

$$
\begin{aligned}
F_{1}(s, z, q) & =\zeta(s)^{z} \prod_{p \mid q}\left(1-p^{-s}\right)^{z} M(q ; s) \prod_{p^{\nu} \| q}\left\{1-\frac{M_{q}(p ; s)}{p-1}\right\} \\
& =\zeta(s)^{z} \prod_{p^{\nu} \| q}\left(1-p^{-s}\right)^{z}\left\{M\left(p^{\nu} ; s\right)-\frac{\tau_{z}\left(p^{\nu-1}\right) p^{-(\nu-1) s}}{p-1}\right\}=\frac{G_{q}(s, z)}{q(s-1)^{z}} .
\end{aligned}
$$

La série $F_{2}$ s'exprime comme combinaison linéaire de puissances $L(s, \chi)^{z}$ où $\chi$ est un caractère non principal de module divisant $q$. Elle possède donc un prolongement analytique à une région du plan complexe débordant dans le demi-plan $\sigma<1$. On 
obtient (6.37) en employant la méthode de Selberg-Delange (cf. [29] chap. II.5). La majoration (6.38) résulte de l'inégalité de Cauchy pour un cercle de centre 1 et de rayon $1 / \log 2 q$. Nous ne détaillons pas les calculs, mais nous mentionnons la majoration

$$
G_{q}(s, z) \ll \tau_{\kappa+1}(q) \log 2 q \quad(|s-1| \ll 1 / \log 2 q) .
$$

Nous déduisons maintenant de ce qui précède une estimation de $Z_{\tau_{z}}(x, x ; \vartheta)$ en fonction des approximations rationnelles de $\vartheta$.

Lemme 6.11. Soient $\varepsilon>0, \kappa \geqslant 1, A>0$ et $B \geqslant 4 A+4 \kappa^{2}+12$. On a uniformément pour $\vartheta \in \mathbb{R}, z \in \mathbb{C},|z| \leqslant \kappa, x \geqslant 2, Q=Q_{x}:=x /(\log x)^{B}, q=q\left(\vartheta ; Q_{x}\right),(a, q)=1$, $|q \vartheta-a| \leqslant 1 / Q_{x}, \vartheta_{q}:=\vartheta-a / q \neq 0$,

$$
\begin{aligned}
Z_{\tau_{z}}(x, x ; \vartheta)= & \frac{x(\log x)^{z-1}}{q}\left\{\frac{\sin ^{2}\left(\pi \vartheta_{q} x\right) \lambda_{0}(z, q)}{\pi \vartheta_{q} x}+O\left(\frac{q^{\varepsilon} \log \left(1+\vartheta_{q}^{2} x^{2}\right)}{\vartheta_{q} x \log x}\right)\right\} \\
& +O\left(\frac{x}{(\log x)^{A}}\right) .
\end{aligned}
$$

Démonstration. Le Lemme 6.8 nous permet de supposer que l'on a $q \leqslant(\log x)^{B}$ : dans le cas contraire, $E_{\tau_{z}}(x, x ; \vartheta)$, et donc $Z_{\tau_{z}}(x, x ; \vartheta)$, est majoré par le terme d'erreur de (6.41), qui domine alors le terme principal.

On a

$$
E_{\tau_{z}}(x, x ; \vartheta)-E_{\tau_{z}}(\sqrt{x}, \sqrt{x} ; \vartheta)=\int_{\sqrt{x}}^{x} e\left(\vartheta_{q} t\right) \mathrm{d} E(t, t ; a / q) .
$$

Notons $R(t ; a / q)$ le terme d'erreur de la formule $(6 \cdot 37)$ pour $J=[B]+1$. Une intégration par parties permet d'écrire

$$
\begin{aligned}
\int_{\sqrt{x}}^{x} e\left(\vartheta_{q} t\right) & \mathrm{d} R(t ; a / q) \\
& \ll|R(x ; a / q)|+|R(\sqrt{x} ; a / q)|+\left|\vartheta_{q}\right| \int_{\sqrt{x}}^{x}|R(u ; a / q)| \mathrm{d} u \\
& \ll \frac{x}{(\log x)^{A}} .
\end{aligned}
$$

De $(6 \cdot 42),(6 \cdot 43)$ et $(6 \cdot 37)$, on déduit donc que

$$
Z_{\tau_{z}}(x, x ; \vartheta)=\sum_{j=0}^{J} \frac{\lambda_{j}(z, q)}{q} \int_{\sqrt{x}}^{x} \sin \left(2 \pi \vartheta_{q} t\right) \mathrm{d}\left\{t(\log t)^{z-1-j}\right\}+O\left(\frac{x}{(\log x)^{A}}\right) .
$$


Admettant momentanément l'évaluation

$$
\begin{aligned}
& \int_{\sqrt{x}}^{x} \sin \left(2 \pi \vartheta_{q} t\right) d\left\{t(\log t)^{z-1-j}\right\} \\
&=x(\log x)^{z-1-j}\left\{\frac{\sin ^{2}\left(\pi \vartheta_{q} x\right)}{\pi \vartheta_{q} x}+O\left(\frac{\log \left(1+\vartheta_{q}^{2} x^{2}\right)}{\vartheta_{q} x \log x}\right)\right\},
\end{aligned}
$$

nous sommes en mesure de conclure grâce aux majorations $(6 \cdot 38)$.

Il reste à prouver $(6 \cdot 44)$. Cette estimation est une conséquence immédiate de la formule

$$
\int_{\sqrt{x}}^{x} \sin (2 \pi \vartheta t)(\log t)^{w} \mathrm{~d} t=x(\log x)^{w}\left\{\frac{\sin ^{2}(\pi \vartheta x)}{\pi \vartheta x}+O\left(\frac{\log \left(1+\vartheta^{2} x^{2}\right)}{\vartheta x \log x}\right)\right\}
$$

valable pour chaque $w \in \mathbb{C}$ fixé et uniformément pour $x \geqslant 2, \vartheta \in \mathbb{R}^{*}$. Pour établir (6·45), nous effectuons d'abord une intégration par parties pour écrire le membre de gauche sous la forme

$$
\left[(\log t)^{w} \frac{\sin ^{2}(\pi \vartheta t)}{\pi \vartheta}\right]_{\sqrt{x}}^{x}-w \int_{\sqrt{x}}^{x} \frac{\sin ^{2}(\pi \vartheta t)}{\pi \vartheta} \frac{(\log t)^{w-1}}{t} \mathrm{~d} t
$$

et nous notons que la majoration

$$
\int_{\sqrt{x}}^{x} \sin ^{2}(\pi \vartheta t) \frac{\mathrm{d} t}{t} \leqslant \int_{0}^{|\vartheta| x}(\sin v)^{2} \frac{\mathrm{d} v}{v} \ll \log \left(1+\vartheta^{2} x^{2}\right)
$$

permet d'estimer convenablement l'intégrale. Il reste à observer, en comparant $|\vartheta|$ à $1 / x$ et à $1 / \sqrt{x \log x}$, que

$$
\frac{\sin ^{2}(\pi \vartheta \sqrt{x})}{\vartheta} \ll \min \left(\frac{1}{|\vartheta|},|\vartheta| x\right) \ll \frac{\log \left(1+\vartheta^{2} x^{2}\right)}{\vartheta \log x} .
$$

\section{7. $P$-convergence de $V(\mu ; \vartheta)$ : preuve du Théorème 2.1}

\section{\%.1. Estimation de $W_{\mu}(x, y ; \vartheta)$}

Étant donnée une fonction arithmétique $f$, nous posons pour $x \geqslant 2, y \geqslant 2, \vartheta \in \mathbb{R}$,

$$
W_{f}(x, y ; \vartheta):=\sum_{n \in S(x, y)} f(n) B(n \vartheta) .
$$

Pour chaque $\varepsilon \in] 0,1[$, nous notons encore

$$
L_{\varepsilon}(v):=\mathrm{e}^{(\log 2 v)^{3(1-\varepsilon) / 5}} \quad(v \geqslant 1)
$$

et

$$
K_{\varepsilon}(x, y):=\frac{1}{\log y}+\frac{1}{L_{\varepsilon}(x / y)} \quad(x \geqslant y \geqslant 2) .
$$

Notre preuve de l'estimation $(2 \cdot 3)$ du Théorème 2.1 repose sur le résultat suivant. 
Proposition 7.1. Soit $A>0$. On a uniformément sous les conditions $x \geqslant y \geqslant 2$, $Q:=x /(\log x)^{5 A+20}, \vartheta \in \mathbb{R}, q=q(\vartheta ; Q)$,

$$
W_{\mu}(x, y ; \vartheta) \ll \Psi(x, y) \frac{q \tau(q)^{2} K_{\varepsilon}(x, y)}{\varphi(q)^{2} \log y}+\frac{x}{(\log x)^{A}} .
$$

En fait, une estimation sensiblement plus précise que $(7 \cdot 3)$ pourrait être obtenue en utilisant notamment les résultats de [2]. Nous nous sommes cantonnés à l'énoncé le plus simple qui permet de déduire le Théorème 2.1 .

Nous dégageons les ingrédients essentiels nécessaires à la preuve de $(7 \cdot 3)$ sous forme de cinq lemmes. Le premier, qui repose sur un argument bien connu, permet de disposer du cas où $\vartheta$ possède une bonne approximation rationnelle avec un 《grand» dénominateur. En vue d'applications ultérieures, dont certaines sont d'ailleurs développées le présent travail, nous donnons une formulation plus générale que nécessaire pour prouver $(7 \cdot 3)$. On pourrait en fait étendre encore davantage la généralité en remplaçant dans l'énoncé la fonction $\tau_{z}$ par une fonction quelconque de $\mathcal{L}^{1}\left(\mathbb{N}^{*}\right)$ satisfaisant une majoration de type $(6 \cdot 32)$.

Lemme 7.2. Soient $\kappa \geqslant 1$ et $R \geqslant 1$. Si $q(\vartheta ; x / R) \geqslant R$, on a uniformément pour $x \geqslant y \geqslant 2, z \in \mathbb{C},|z| \leqslant \kappa, \vartheta \in \mathbb{R}$,

$$
W_{\tau_{z}}(x, y ; \vartheta) \ll x(\log x)^{\kappa^{2}+3} \varepsilon(R, x)
$$

où l'on a posé $\varepsilon(R, x):=R^{-1 / 5}+\mathrm{e}^{-\frac{1}{2} \sqrt{\log x}}$.

Démonstration. Le principe de la preuve consiste à approcher $B(\vartheta)$ par une série de Fourier absolument convergente et à appliquer l'estimation du Lemme 6.8 pour $E_{\tau_{z}}(x, y ; \vartheta)$.

Soit $\delta$ un paramètre positif que nous choisirons ultérieurement. Nous approchons $B(\vartheta)$ par

$$
b_{\delta}(\vartheta):=\frac{1}{\delta} \int_{-\delta / 2}^{\delta / 2} B(\vartheta-u) \mathrm{d} u= \begin{cases}B(\vartheta) & \text { si }\|\vartheta\|>\frac{1}{2} \delta \\ \langle\vartheta\rangle-\langle\vartheta\rangle / \delta & \text { si }\|\vartheta\| \leqslant \frac{1}{2} \delta\end{cases}
$$

On note que $b_{\delta}$ est 1-périodique, continue et impaire. Posons

$$
b_{\delta}^{*}(\vartheta):=\left|B(\vartheta)-b_{\delta}(\vartheta)\right|=\frac{1}{2}(1-2\|\vartheta\| / \delta)^{+},
$$

de sorte que

$$
W_{\tau_{z}}(x, y ; \vartheta)=\sum_{n \in S(x, y)} \tau_{z}(n) b_{\delta}(n \vartheta)+O\left(\sum_{n \in S(x, y)} \tau_{\kappa}(n) b_{\delta}^{*}(n \vartheta)\right)
$$


où l'on a utilisé la majoration $\left|\tau_{z}(n)\right| \leqslant \tau_{\kappa}(n)(n \geqslant 1)$. On a les développements en séries de Fourier

$$
b_{\delta}(\vartheta)=\sum_{m=1}^{\infty} b_{\delta, m} \sin (2 \pi m \vartheta), \quad b_{\delta}^{*}(\vartheta)=\sum_{m=0}^{\infty} a_{\delta, m} \cos (2 \pi m \vartheta),
$$

avec

$$
\left\{\begin{array}{l}
b_{\delta, m}:=-\frac{\sin (\pi m \delta)}{\pi^{2} m^{2} \delta} \quad(m \geqslant 1), \\
a_{\delta, 0}:=\frac{1}{4} \delta, \quad a_{\delta, m}:=\frac{2 \sin ^{2}(\pi m \delta / 2)}{\pi^{2} m^{2} \delta} \quad(m \geqslant 1) .
\end{array}\right.
$$

En particulier

$$
\left|b_{\delta, m}\right| \ll \frac{1}{m(1+\delta m)} \quad(m \geqslant 1), \quad\left|a_{\delta, m}\right| \ll \frac{\delta}{1+m^{2} \delta^{2}} \quad(m \geqslant 0),
$$

d'où

$$
\sum_{m \geqslant 0}\left|a_{\delta, m}\right| \ll 1, \quad \sum_{m>M}\left(\left|b_{\delta, m}\right|+\left|a_{\delta, m}\right|\right) \ll 1 /(\delta M) \quad(M \geqslant 1) .
$$

Nous choisissons $M=R / 5$ et imposons $R \delta \geqslant 1$. Une interversion de sommations permet d'écrire

$$
\begin{aligned}
\sum_{n \in S(x, y)} \tau_{z}(n) b_{\delta}(n \vartheta) & =\sum_{m=1}^{\infty} b_{\delta, m} Z_{\tau_{z}}(x, y ; m \vartheta) \\
& =\sum_{m \leqslant M} b_{\delta, m} Z_{\tau_{z}}(x, y ; m \vartheta)+O\left(\frac{x}{R \delta}(\log x)^{\kappa-1}\right) .
\end{aligned}
$$

Nous pouvons supposer sans perte de généralité que $3 R^{3} \leqslant x$. Posons $Q:=x / R$. Le Lemme 6.2 implique

$$
q(m \vartheta ; 2 Q) \geqslant q(\vartheta ; Q) / 2 m \geqslant R / 2 m \quad(1 \leqslant m \leqslant M) .
$$

Il vient grâce au Lemme 6.8

$$
\begin{aligned}
\sum_{m \leqslant M} b_{\delta, m} Z_{\tau_{z}}(x, y ; m \vartheta) \ll x(\log x)^{3+\kappa^{2}} \sum_{m \leqslant M}\left|b_{\delta, m}\right|\left\{\left(\frac{m}{R}\right)^{1 / 4}+\mathrm{e}^{-\sqrt{\log x}}\right\} \\
\ll x \frac{(\log x)^{3+\kappa^{2}}}{R^{1 / 4}}\left(\sum_{m \leqslant 1 / \delta} \frac{1}{m^{3 / 4}}+\sum_{1 / \delta<m \leqslant M} \frac{1}{\delta m^{7 / 4}}\right)+x \frac{(\log x)^{4+\kappa^{2}}}{\mathrm{e}^{\sqrt{\log x}}} \\
\ll x(\log x)^{3+\kappa^{2}}\left(\frac{1}{R^{1 / 4} \delta^{1 / 4}}+\mathrm{e}^{-\frac{1}{2} \sqrt{\log x}}\right) .
\end{aligned}
$$


Nous déduisons donc que

$$
\sum_{n \in S(x, y)} \tau_{z}(n) b_{\delta}(n \vartheta) \ll x(\log x)^{3+\kappa^{2}}\left(\frac{1}{R^{1 / 4} \delta^{1 / 4}}+\mathrm{e}^{-\frac{1}{2} \sqrt{\log x}}\right) .
$$

Notant $K_{\tau_{\kappa}}(x, y ; \vartheta):=\sum_{n \in S(x, y)} \cos (2 \pi n \vartheta)$, nous avons de même

$$
\begin{aligned}
\sum_{n \in S(x, y)} & \tau_{\kappa}(n) b_{\delta}^{*}(n \vartheta)=\sum_{m=0}^{\infty} a_{\delta, m} K_{\tau_{\kappa}}(x, y ; m \vartheta) \\
& =\sum_{1 \leqslant m \leqslant M} a_{\delta, m} K_{\tau_{\kappa}}(x, y ; m \vartheta)+O\left(x(\log x)^{\kappa-1}\{\delta+1 /(R \delta)\}\right) \\
& \ll x(\log x)^{3+\kappa^{2}}\left(\frac{1}{R^{1 / 4} \delta^{1 / 4}}+\mathrm{e}^{-\frac{1}{2} \sqrt{\log x}}\right)+x(\log x)^{\kappa-1} \delta
\end{aligned}
$$

où l'on a utilisé l'inégalité $R \delta \geqslant 1$.

Reportons $(7 \cdot 10)$ et $(7 \cdot 11)$ dans $(7 \cdot 5)$ pour le choix $\delta:=R^{-1 / 5}$. Nous obtenons bien $(7 \cdot 4)$.

Les deux lemmes suivants sont dévolus à mettre en place des outils généraux pour estimer $W_{f}(x, x ; \vartheta)$. Nous en déduirons ensuite la majoration requise en choisissant $f=\mu \mathbf{1}_{S(\infty, y)}$.

Notre approche s'applique aux fonctions arithmétiques qui sont bien réparties dans les progressions arithmétiques de «petit» module. Nous formalisons la notion sous-jacente dans la définition suivante.

Définition 7.3. On dit qu'une fonction arithmétique $f$ est de type Siegel-Walfisz fort, et l'on note $f \in S W^{+}$, si l'une des deux conditions équivalentes suivantes est satisfaite :

(i) pour tout $A>0$ fixé, et uniformément pour $r \in \mathbb{N}^{*}, m \in \mathbb{N}^{*}, c \in \mathbb{N}^{*}$, $(c, m)=1$, on a

$$
\sum_{\substack{n \leqslant x / r \\ n \equiv c(\bmod m)}} f(r n)=\frac{1}{\varphi(m)} \sum_{\substack{n \leqslant x / r \\(n, m)=1}} f(r n)+O\left(\frac{x}{(\log x)^{A}}\right) ;
$$

(ii) pour tout $A>0$ fixé, et uniformément pour $q \in \mathbb{N}^{*}, a \in \mathbb{Z}$, on a

$$
\sum_{\substack{n \leqslant x \\ n \equiv a(\bmod q)}} f(n)=\frac{1}{\varphi(m)} \sum_{\substack{n \leqslant m x / q \\(n, m)=1}} f(q n / m)+O\left(\frac{x}{(\log x)^{A}}\right),
$$

où l'on a posé $m:=q /(q, a)$.

L'équivalence entre ces deux assertions résulte d'un simple changement de variable dans les sommations. Des notions analogues ont été utilisées dans de 
nombreux travaux. Ainsi, par exemple, les fonctions de type Siegel-Walfisz fort satisfont à la condition (i) du théorème 1 de [13].

Nous montrons aux Lemmes 7.6 et 13.5 que les fonctions $\mathbf{1}_{S(\infty, y)} \mu$ et $\tau_{z}$ sont de type Siegel-Walfisz fort, les constantes impliquées dans (7.12) et (7.13) étant uniformes en $y$ et localement uniformes en $z$.

Lorsque $(a, q)=1$ et $m \mid q$, la quantité

$$
\gamma(m ; b / q):=\sum_{\substack{1 \leqslant d \leqslant m \\(d, m)=1}} B\left(\frac{a d}{m}+\frac{b}{q}\right)
$$

est clairement indépendante de $a$. L'énoncé suivant fournit une majoration.

Lemme 7.4. Soient $q \in \mathbb{N}^{*}, m \mid q$. On a

$$
\sup _{b \in \mathbb{Z}}|\gamma(m ; b / q)| \leqslant \tau(m)+\frac{1}{2} .
$$

Démonstration. On ne réduit pas la généralité en supposant $0 \leqslant b<q$ et, ainsi que nous l'avons remarqué, $a=1$. On note également que $\gamma(m ; 0)=0$ en vertu de la symétrie autour de $m / 2$ des entiers premiers à $m$. On a

$$
B\left(\frac{d}{m}+\frac{b}{q}\right)=B\left(\frac{d}{m}\right)+\frac{b}{q}-X\left(\frac{d}{m}+\frac{b}{q}\right) \quad \text { où } \quad X(v):= \begin{cases}0 & \text { si } 0<v<1, \\ \frac{1}{2} & \text { si } v=1, \\ 1 & \text { si } 1<v<2 .\end{cases}
$$

Il s'ensuit que

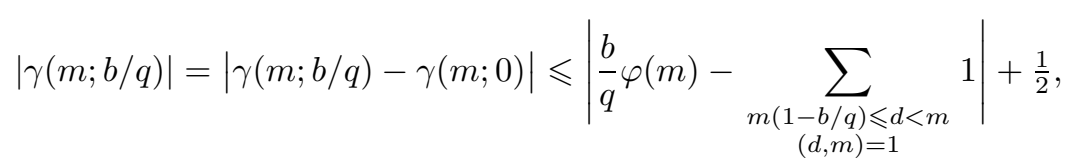

où le terme $\frac{1}{2}$ dans le membre de droite prend en compte l'éventualité $d / m+b / q=1$. Le changement de variable $d \mapsto m-d$ permet de récrire la somme en $d$ sous la forme

$$
\sum_{\substack{d \leqslant b m / q \\(d, m)=1}} 1=\sum_{d \leqslant b m / q} \sum_{c \mid(d, m)} \mu(c)=\sum_{c \mid m} \mu(c)\left[\frac{b m}{q c}\right]=\frac{b \varphi(m)}{q}-\sum_{c \mid m} \mu(c)\left\langle\frac{b m}{q c}\right\rangle,
$$

où nous avons utilisé la formule $\varphi(m) / m=\sum_{c \mid m} \mu(c) / c$. Cela implique bien (7·15). 
L'énoncé du lemme suivant nécessite quelques notations. Lorsque $\vartheta \in \mathbb{R} \backslash \mathbb{Q}$ et $b \geqslant 0$, nous posons $x_{b}:=\min (x, b /\|q \vartheta\|)$ et introduisons, étant donnée une fonction arithmétique $f$, les sommes

$$
\begin{aligned}
& T_{b}^{(1)}=T_{b}^{(1)}(x ; \vartheta):=\sum_{x_{b}<n \leqslant x_{b+1}} f(n) B\left(\frac{a n+b}{q}\right), \\
& T_{b}^{(2)}=T_{b}^{(2)}(x ; \vartheta):=\sum_{x_{b}<n \leqslant x_{b+1}} f(n)\left(n \vartheta_{q}-\frac{b}{q}\right), \\
& T_{b}^{(3)}=T_{b}^{(3)}(x ; \vartheta):=\sum_{\substack{x_{b}<n \leqslant x_{b+1} \\
a n \equiv-b(\bmod q)}} f(n) .
\end{aligned}
$$

Lemme 7.5. Soient $A, B>0$. On a uniformément pour $f \in S W^{+}, \vartheta \in \mathbb{R} \backslash \mathbb{Q}$, $x \geqslant 2, Q=Q_{x}:=x /(\log x)^{B}, q=q\left(\vartheta ; Q_{x}\right) \leqslant(\log x)^{B}, a \in \mathbb{Z},(a, q)=1$, $|q \vartheta-a| \leqslant 1 / Q_{x}, \vartheta_{q}:=\vartheta-a / q>0$,

$$
W_{f}(x, x ; \vartheta)=\sum_{0 \leqslant b \leqslant x\|q \vartheta\|} T_{b}+O\left((\log x)^{B} N_{f}(x)\right)
$$

où $N_{f}(x):=\max _{n \leqslant x}|f(n)|$ et

$$
T_{b}:=T_{b}^{(1)}+T_{b}^{(2)}-\frac{1}{2} T_{b}^{(3)} .
$$

De plus, on a sous les mêmes conditions, et uniformément pour $0 \leqslant b \leqslant x\|q \vartheta\|$,

$$
\begin{aligned}
& T_{b}^{(1)}=\sum_{m \mid q} \frac{\gamma(m ; b / q)}{\varphi(m)} \sum_{\substack{x_{b} m / q<\ell \leqslant x_{b+1} m / q \\
(\ell, m)=1}} f\left(\frac{q \ell}{m}\right)+O\left(\frac{x}{(\log x)^{B+A}}\right), \\
& T_{b}^{(3)}=\frac{1}{\varphi(d)} \sum_{\substack{d x_{b} / q<\ell \leqslant d x_{b+1} / q \\
(\ell, d)=1}} f\left(\frac{q \ell}{d}\right)+O\left(\frac{x}{(\log x)^{B+A}}\right),
\end{aligned}
$$

où l'on a noté $d=q /(b, q)$ et où les constantes implicites ne dépendent que de celles de $(7 \cdot 12)$ et $(7 \cdot 13)$.

Remarque. Puisque $x\|q \vartheta\| \ll(\log x)^{B}$, la contribution à (7.17) des termes d'erreur de $(7 \cdot 19)$ est toujours $\ll x /(\log x)^{A}$.

Démonstration. Soit $T_{b}^{*}$ la sous-somme de $W_{f}(x, x ; \vartheta)$ correspondant à la condition supplémentaire $x_{b}<n \leqslant x_{b+1}$. Lorsque $n$ est compté dans $T_{b}^{*}$, on a donc $b / q<n \vartheta_{q} \leqslant(b+1) / q$, où la seconde inégalité est stricte sauf pour au plus une valeur de $n$. Lorsque $x_{b}<n<x_{b+1}$, on a la décomposition

$$
B(n \vartheta)=B\left(n a / q+n \vartheta_{q}\right)=B\left(\frac{a n+b}{q}\right)+\left(n \vartheta_{q}-\frac{b}{q}\right)-\frac{1}{2} \mathbf{1}_{\mathbb{Z}}\left(\frac{a n+b}{q}\right) .
$$


Il s'ensuit que

$$
T_{b}^{*}=T_{b}+O\left(N_{f}(x)\right) \quad(0 \leqslant b \leqslant x\|q \vartheta\|),
$$

et l'on note immédiatement que, puisque $\|q \vartheta\| \leqslant 1 / Q \leqslant 2(\log x)^{B} / x$, la contribution globale à $(7 \cdot 17)$ des termes d'erreur de $(7 \cdot 21)$ est $\ll(\log x)^{B} N_{f}(x)$. Cela établit bien $(7 \cdot 17)$.

Il reste à établir les estimations $(7 \cdot 19)$.

Classons les entiers $n$ comptés dans $T_{b}^{(1)}$ selon leurs résidus modulo $q$, paramétrés sous la forme $c q / m(\bmod q)$ où $m \mid q$ et $(c, m)=1$. Nous obtenons la décomposition

$$
T_{b}^{(1)}=\sum_{m \mid q} \sum_{\substack{1 \leqslant c \leqslant m \\(c, m)=1}} B\left(\frac{c a}{m}+\frac{b}{q}\right) \sum_{\substack{x_{b}<n \leqslant x_{b+1} \\ n \equiv c q / m(\bmod q)}} f(n)
$$

Compte tenu de la notation $(7 \cdot 14)$, nous déduisons donc de $(7 \cdot 13)$ l'estimation requise pour $T_{b}^{(1)}$.

On déduit par ailleurs directement de $(7 \cdot 13)$ que

$$
T_{b}^{(3)}=\frac{1}{\varphi(d)} \sum_{\substack{d x_{b} / q<\ell \leqslant d x_{b+1} / q \\(\ell, d)=1}} f\left(\frac{q \ell}{d}\right)+O\left(\frac{x}{(\log x)^{B+A}}\right),
$$

c'est-à-dire l'évaluation annoncée dans (7·19).

Nos deux derniers lemmes concernent le comportement de la fonction sommatoire de la fonction de Möbius sur l'intersection de $S(x, y)$ et d'une progression arithmétique. Nous posons

$$
M(x, y ; a, q):=\sum_{\substack{n \in S(x, y) \\ n \equiv a(\bmod q)}} \mu(n), \quad M_{q}(x, y):=\sum_{\substack{n \in S(x, y) \\(n, q)=1}} \mu(n)
$$

Lemme 7.6. Soit $A>0$. On a uniformément pour $x \geqslant y \geqslant 2, q \geqslant 1, a \in \mathbb{Z}$, $(a, q)=1$,

$$
M(x, y ; a, q)=\frac{M_{q}(x, y)}{\varphi(q)}+O\left(\frac{x}{(\log x)^{A}}\right)
$$

De plus, notant $\mu_{y}:=\mathbf{1}_{S(\infty, y)} \mu$, on a

$$
\mu_{y} \in S W^{+} \quad(y \geqslant 2),
$$

les constantes implicites de (7-12) et (7-13) pour $f=\mu_{y}$ étant indépendantes de $y$. 
Démonstration. Les estimations triviales

$$
|M(x, y ; a, q)| \leqslant \min \{\Psi(x, y), 1+x / q\} \quad\left|M_{q}(x, y)\right| \leqslant \Psi(x, y),
$$

nous permettent, grâce par exemple à $(6 \cdot 2)$, de restreindre la preuve de $(7 \cdot 24)$ au cas $x^{1 / \log _{2} x}<y \leqslant x, q \leqslant(\log x)^{A}$. La formule requise est donc une conséquence immédiate des évaluations des quantités $(7 \cdot 23)$ établies aux théorèmes 3 et 5 de [2].

Nous allons maintenant déduire, avec l'uniformité annoncée, (7·25) de (7·24).

Observons d'abord que $(7 \cdot 24)$ coïncide avec $(7 \cdot 12)$ lorsque $r=1$. Pour obtenir l'assertion générale, il suffit donc de considérer le cas $1<r \leqslant(\log x)^{A}$ où $A$ est une constante arbitraire positive. Soient $m \geqslant 1, r>1$. Désignons par $r_{m}$ le plus grand diviseur de $r$ premier à $m$. Lorsque $(c, m)=1$, on a

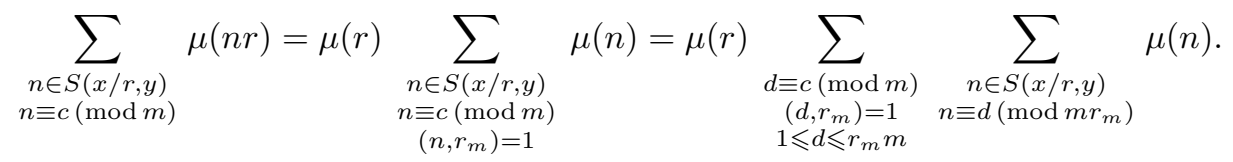

La relation $(7 \cdot 24)$ fournit alors, puisque $r_{m} \leqslant r \leqslant(\log x)^{A}$,

$$
\begin{aligned}
\sum_{\substack{n \in S(x / r, y) \\
n \equiv c(\bmod m)}} \mu(n r) & =\mu(r) \frac{\varphi\left(r_{m}\right)}{\varphi\left(m r_{m}\right)} \sum_{\substack{n \in S(x / r, y) \\
\left(n, m r_{m}\right)=1}} \mu(n)+O\left(\frac{x \varphi\left(r_{m}\right)}{(\log x)^{2 A}}\right) \\
& =\frac{1}{\varphi(m)} \sum_{\substack{n \in S(x / r, y) \\
(n, m)=1}} \mu(n r)+O\left(\frac{x}{(\log x)^{A}}\right) .
\end{aligned}
$$

Lemme 7.7. Soit $A>0$. On a uniformément pour $x \geqslant y \geqslant 2, z \geqslant 1, q \geqslant 1$,

$$
M_{q}(x+x / z, y)-M_{q}(x, y) \ll \frac{q \Psi(x, y) K_{\varepsilon}(x, y)}{z \varphi(q) \log y}+\frac{x}{(\log x)^{A}} .
$$

Démonstration. Comme précédemment, nous pouvons restreindre l'étude au cas

$$
x^{1 / \log _{2} x} \leqslant y \leqslant x, \quad 1 \leqslant z \leqslant(\log x)^{A} .
$$

Posons

$$
M_{q}(x):=\sum_{\substack{n \leqslant x \\(n, q)=1}} \mu(n)
$$

Le théorème 3 de [2] permet d'écrire, dans le domaine requis, ${ }^{(16)}$

$$
M_{q}(x, y)=\int_{0}^{x} \mu_{q}(t, y) \mathrm{d} t+O\left(\frac{x}{(\log x)^{A}}\right)
$$

16. Le résultat de [2] comporte la restriction supplémentaire $q \leqslant y$. On peut vérifier qu'elle est en fait superflue. 
avec

$$
\mu_{q}(t, y):=\int_{0}^{\infty} \omega\left(u_{t, y}-v\right) M_{q}\left(y^{v}\right) y^{-v} \mathrm{~d} v+\frac{y}{\log y} M_{q}(t / y)
$$

où $\omega$ désigne la fonction de Buchstab. ${ }^{(17)}$

La quantité $M_{q}(x)$ est la fonction sommatoire associée à la série de Dirichlet

$$
\zeta(s)^{-1} \prod_{p \mid q}\left(1-p^{-s}\right)^{-1}
$$

Cela implique d'une part

$$
\int_{0}^{\infty} M_{q}\left(y^{v}\right) y^{-v} \mathrm{~d} v=0
$$

et d'autre part, en appliquant par exemple le théorème II.5.5 de [29], pour chaque $\varepsilon>0$,

$$
M_{q}(x) \ll_{\varepsilon} \frac{q x}{\varphi(q) L_{\varepsilon}(x)} \quad(x \geqslant 2, q \geqslant 1) .{ }^{(18)}
$$

Les relations $(7 \cdot 29)$ et $(7 \cdot 30)$ permettent, en raisonnant comme dans [28] où le cas $q=1$ est traité aux pp. 235-6, d'obtenir l'estimation

$$
\mu_{q}(t, y) \ll \frac{q \varrho\left(u_{t, y}\right) K_{\varepsilon}(t, y)}{\varphi(q) \log y} \quad(t \geqslant 2, y \geqslant 2, q \geqslant 1) .
$$

Nous renvoyons le lecteur à [28] pour les détails calculatoires, qui sont essentiellement indépendants de la valeur de $q$. En reportant cette estimation dans $(7 \cdot 28)$, on obtient bien $(7 \cdot 26)$.

Fin de la démonstration de la Proposition 7.1.

Soit $B:=5 A+20$, de sorte que $Q=x /(\log x)^{B}$ et $q=q(\vartheta ; Q)$. Si $q=$ $q(\vartheta ; Q)>(\log x)^{B}$, le Lemme 7.2 , appliqué avec $z=-1$, permet de conclure. Nous pouvons donc supposer dans la suite que $q \leqslant(\log x)^{B}$. Au vu de la majoration triviale $\left|W_{\mu}(x, y ; \vartheta)\right| \leqslant \Psi(x, y)$ et de $(6 \cdot 2)$, nous pouvons également, sans perte de généralité, faire l'hypothèse supplémentaire

$$
x^{1 / \log _{2} x}<y \leqslant x .
$$

17. Voir par exemple [29], chap. III.6.

18. En toute rigueur, le résultat invoqué n'implique cette estimation qu'en y remplaçant $L_{\varepsilon}(x)$ par $\mathrm{e}^{c \sqrt{\log x}}$ avec une constante $c>0$ adéquate. Cela est dû au fait que la démonstration donnée dans [29] n'utilise, pour des raisons de cohérence et de complétude, que la région sans zéro classique de La Vallée-Poussin pour la fonction zêta de Riemann. En incorporant celle de Vinogradov-Korobov, la preuve fournit immédiatement la borne annoncée. On notera que, dans la formule (III.5.30) de [29], toutes les quantités $\lambda_{k}(z)$ sont nulles pour $z=-1$ et que l'on a $H_{N}(-1) \ll(\log 2 q)^{N+3}$. 
Il existe un entier $a$ vérifiant $(a, q)=1$ et tel que, notant $\vartheta_{q}=\vartheta-a / q$, l'on ait $\|q \vartheta\|= \pm q \vartheta_{q}$. Nous supposons, pour fixer les idées, que $\vartheta_{q}>0$.

Le Lemme 7.6 nous permet d'appliquer le Lemme 7.5 aux fonctions $\mu_{y}=\mu \mathbf{1}_{S(\infty, y)}$ avec des constantes implicites indépendantes de $y$. Avec les notations $(7 \cdot 16)$, on peut donc écrire

$$
W_{\mu}(x, y ; \vartheta)=\sum_{0 \leqslant b \leqslant x\|q \vartheta\|} T_{b}+O\left((\log x)^{B}\right) .
$$

On a

$$
\sum_{\substack{\ell \in S(x, y) \\(\ell, m)=1}} \mu(q \ell / m)=\mu(q / m) M_{q}(x, y) .
$$

La conjonction des Lemmes 7.4, 7.5 et 7.7 fournit donc

$$
\begin{aligned}
T_{b}^{(1)} & \ll \sum_{m \mid q} \frac{|\gamma(m ; b / q)|}{\varphi(m)}\left|M_{q}\left(\frac{m x_{b+1}}{q}, y\right)-M_{q}\left(\frac{m x_{b}}{q}, y\right)\right|+\frac{x}{(\log x)^{B+A}} \\
& \ll \frac{K_{\varepsilon}\left(x_{b} / q, y\right)}{b \log y} \sum_{m \mid q} \frac{\tau(m) q}{\varphi(m) \varphi(q)} \Psi\left(\frac{x_{b} m}{q}, y\right)+\frac{x}{(\log x)^{B+A}} .
\end{aligned}
$$

En observant que, dans le domaine $(7 \cdot 27)$ et pour $1 \leqslant q \leqslant(\log x)^{B}$,

$$
K_{\varepsilon}\left(x_{b} / q, y\right) \ll K_{\varepsilon}\left(x_{b}, y\right), \quad \Psi\left(\frac{x_{b} m}{q}, y\right) \ll \frac{m}{q} \Psi\left(x_{b}, y\right) \quad(m \mid q),
$$

on en déduit que

$$
\begin{aligned}
T_{b}^{(1)} & \ll \frac{q \tau(q)^{2} \Psi\left(x_{b}, y\right) K_{\varepsilon}\left(x_{b}, y\right)}{b \varphi(q)^{2} \log y}+\frac{x}{(\log x)^{B+A}} \\
& \ll \frac{q \tau(q)^{2} \Psi(x, y) K_{\varepsilon}(x, y)}{x\|q \vartheta\| \varphi(q)^{2} \log y}+\frac{x}{(\log x)^{B+A}},
\end{aligned}
$$

où la seconde évaluation résulte, compte tenu de $(7 \cdot 32)$, de propriétés bien connues du comportement local de $\Psi(x, y)$.

Semblablement, le Lemme 7.7 appliqué avec $q=1$ fournit par sommation d'Abel

$$
\begin{aligned}
T_{b}^{(2)} & \ll \frac{\Psi\left(x_{b}, y\right) K_{\varepsilon}\left(x_{b}, y\right)}{b q \log y}+\frac{x}{(\log x)^{B+A}} \\
& \ll \frac{\Psi(x, y) K_{\varepsilon}(x, y)}{x\|q \vartheta\| q \log y}+\frac{x}{(\log x)^{B+A}} .
\end{aligned}
$$

Enfin, nous déduisons de $(7 \cdot 33)$ et de $(7 \cdot 26)$ que

$$
\begin{aligned}
T_{b}^{(3)} & \ll \frac{q \Psi\left(x_{b} / d, y\right) K_{\varepsilon}\left(x_{b} / d, y\right)}{d \varphi(q / d)^{2} b \log y}+\frac{x}{(\log x)^{B+A}} \\
& \ll \frac{q^{2} \Psi(x, y) K_{\varepsilon}(x, y)}{x\|q \vartheta\| q \varphi(q)^{2} \log y}+\frac{x}{(\log x)^{B+A}} .
\end{aligned}
$$

En reportant $(7 \cdot 34),(7 \cdot 35)$ et $(7 \cdot 36)$ dans $(7 \cdot 18)$ puis $(7 \cdot 17)$, nous obtenons bien l'estimation requise $(7 \cdot 3)$. 


\subsection{Estimation de $W_{\mu}(x, y ; a / q)$}

Nous rassemblons ici deux résultats auxiliaires nécessaires à la preuve de (2·4).

Lemme 7.8. Soit $A>0$. On a uniformément pour $x \geqslant y \geqslant 2, q \in \mathbb{N}$, $1 \leqslant q \leqslant(\log x)^{A}, a \in \mathbb{Z},(a, q)=1$,

$$
Z_{\mu}(x, y ; a / q) \ll \frac{x}{(\log x)^{A}}
$$

Démonstration. Cette majoration est une conséquence immédiate de l'estimation de $E_{\mu}(x, y ; a / q)$ donnée au théorème 6 de [2] et dont le terme principal, réel, ne contribue pas à $Z_{\mu}(x, y ; a / q)$.

Lemme 7.9. Soient $A>0, B:=5 A+20$. On a uniformément pour $x \geqslant y \geqslant 2$, $q \in \mathbb{N}, 1 \leqslant q \leqslant x /(\log x)^{B}, a \in \mathbb{Z},(a, q)=1$,

$$
W_{\mu}(x, y ; a / q) \ll \frac{x}{(\log x)^{A}}
$$

Démonstration. L'inégalité triviale $\left|W_{\mu}(x, y ; a / q)\right| \leqslant \Psi(x, y)$ nous permet, compte tenu de $(6 \cdot 2)$, de restreindre l'étude au cas $y>x^{1 / \log _{2} x}$. D'après le Lemme 7.2 , la majoration (7.38) a bien lieu si $q>(\log x)^{B}$. Dans le cas contraire, nous allons montrer que le résultat souhaité est une conséquence de (7·37).

Pour tout $a \in \mathbb{Z}$, la relation suivante résulte du calcul des coefficients de Fourier discrets de la fonction $q$-périodique $n \mapsto B(n / q)$ : ${ }^{(19)}$

$$
\begin{aligned}
B\left(\frac{a}{q}\right) & =-\frac{1}{2 q} \sum_{1 \leqslant b<q} \cot \left(\frac{\pi b}{q}\right) \sin \left(\frac{2 \pi b a}{q}\right) \\
& =-\frac{1}{2 q} \sum_{m \mid q} \sum_{\substack{1 \leqslant c<m \\
(c, m)=1}} \cot \left(\frac{\pi c}{m}\right) \sin \left(\frac{2 \pi c a}{m}\right) .
\end{aligned}
$$

Nous en déduisons que

$$
W_{\mu}(x, y ; a / q)=-\frac{1}{2 q} \sum_{m \mid q} \sum_{\substack{1 \leqslant c<m \\(c, m)=1}} \cot \left(\frac{\pi c}{m}\right) Z_{\mu}(x, y, a c / m) .
$$

\footnotetext{
19. On sait a priori qu'il existe des coefficients $c_{b}$ tels que $B(a / q)=\sum_{1 \leqslant b<q} c_{b} \sin (2 \pi b a / q)$.

L'orthogonalité des caractères additifs implique donc

$$
c_{b}=\frac{1}{q} \sum_{1 \leqslant k<q} B(k / q) \sin (2 \pi b k / q)=\frac{1}{q^{2}} \sum_{1 \leqslant k<q} k \sin (2 \pi b k / q) \quad(1 \leqslant b<q) .
$$

La relation $\sum_{1 \leqslant k<q} k z^{k}=z\left\{1+(q-1) z^{q}-q z^{q-1}\right\} /(1-z)^{2}(z \neq 1)$, appliquée à $z=\exp (2 \pi i b / q)$ fournit alors $c_{b}=-(1 / 2 q) \cot (\pi b / q)$.
} 
On conclut donc en appliquant (7.37) et en remarquant que

$$
\sum_{\substack{1 \leqslant c<m \\(c, m)=1}}\left|\cot \left(\frac{\pi c}{m}\right)\right| \ll \varphi(m) \log m .
$$

En effet

$$
\begin{aligned}
\sum_{\substack{1 \leqslant c<m \\
(c, m)=1}}\left|\cot \left(\frac{\pi c}{m}\right)\right| & \leqslant \sum_{\substack{1 \leqslant c \leqslant m / 2 \\
(c, m)=1}} \frac{m}{c} \\
& \leqslant m \sum_{1 \leqslant c<m} \frac{1}{c} \sum_{d \mid(c, m)} \mu(d)=m \sum_{d \mid m} \frac{\mu(d)}{d} \sum_{t<m / d} \frac{1}{t} \\
& =m \sum_{d \mid m} \frac{\mu(d)}{d}\left\{\log (m / d)+\gamma+O\left(\frac{d}{m}\right)\right\} \\
& =\varphi(m)\left\{\log m+\gamma-\sum_{p \mid m} \frac{\log p}{p-1}\right\}+O\left(2^{\omega(m)}\right),
\end{aligned}
$$

où nous avons calculé la somme $\sum_{d \mid m} \mu(d)(\log d) / d$ par dérivation logarithmique du produit $\prod_{p \mid m}\left(1-p^{-s}\right)$ en $s=1$.

\subsection{Preuve du Théorème 2.1}

Grâce à la convergence de la série $\sum_{n=1}^{\infty} \mu(n) B(n \vartheta) / n$, nous pouvons écrire

$$
\nabla(\vartheta ; y)=-\sum_{P(n)>y} \frac{\mu(n) B(n \vartheta)}{n}=\int_{y}^{\infty} \frac{W_{\mu}(x, x ; \vartheta)-W_{\mu}(x, y ; \vartheta)}{x^{2}} \mathrm{~d} x .
$$

Soit $A>0$. Posant $Q_{y}:=y /(\log y)^{5 A+20}, q:=q\left(\vartheta ; Q_{y}\right)$, on a

$$
\begin{aligned}
\int_{y}^{+\infty} W_{\mu}(x, x ; \vartheta) \frac{\mathrm{d} x}{x^{2}} & =\frac{W_{\mu}(y, y ; \vartheta)}{y}-\sum_{n>y} \frac{\mu(n) B(n \vartheta)}{n} \\
& \ll \frac{q \tau(q)^{2}}{\varphi(q)^{2} \log y}+\frac{1}{(\log y)^{A}},
\end{aligned}
$$

d'après $(7 \cdot 3)$ et $(1 \cdot 7)$.

Nous avons ensuite par $(7 \cdot 3)$

$$
\begin{aligned}
\int_{y}^{\infty} \frac{W_{\mu}(x, y ; \vartheta)}{x^{2}} \mathrm{~d} x & \ll \int_{y}^{\infty} \frac{q \tau(q)^{2} \Psi(x, y) K_{\varepsilon}(x, y)}{x^{2} \varphi(q)^{2} \log y} \mathrm{~d} x+\frac{1}{(\log y)^{A}} \\
& \ll \frac{q \tau(q)^{2}}{\varphi(q)^{2} \log y}+\frac{1}{(\log y)^{A}} .
\end{aligned}
$$

Nous avons donc bien établi $(2 \cdot 3)$.

La relation (2.4) résulte immédiatement de (7.38). La preuve du Théorème 2.1 est donc complète. 


\section{8. $\boldsymbol{P}$-convergence de $U(1 ; \vartheta)$ : preuve du Théorème 2.2}

Nous montrons d'abord trois résultats préliminaires faciles.

Lemme 8.1. Pour tous $\vartheta \in \mathbb{R}, y \geqslant 2$, on a

$$
\nabla_{\mathbf{1}}(\vartheta ; y)=\int_{2}^{\infty} \frac{Z(x, x ; \vartheta)-Z(x, y ; \vartheta)}{\pi x^{2}} \mathrm{~d} x .
$$

Démonstration. La convergence de la série $B(\vartheta)$ permet d'écrire

$$
\nabla_{\mathbf{1}}(\vartheta ; y)=\sum_{P(n)>y} \frac{\sin (2 \pi n \vartheta)}{-\pi n}=\int_{y}^{\infty} \frac{\mathrm{d}\{Z(x, x ; \vartheta)-Z(x, y ; \vartheta)\}}{-\pi x}
$$

La formule requise (8.1) s'en déduit par sommation d'Abel, puisque l'intégrande est nul pour $2 \leqslant x \leqslant y$.

Lemme 8.2. On a uniformément pour $x \geqslant 2, y \geqslant 2$,

$$
\int_{x}^{\infty} \frac{\Psi(t, y)}{t^{2}} \mathrm{~d} t \ll(\log y) u^{-u}+x^{-3 / 4}
$$

Démonstration. Cela découle immédiatement de $(6 \cdot 2)$.

Lemme 8.3. On a uniformément pour $\vartheta \in \mathbb{R} \backslash \mathbb{Z}$ et $T \geqslant 1$

$$
B(\vartheta)=\int_{1 /(T\|\vartheta\|)}^{T /\|\vartheta\|} \frac{Z(x, x ; \vartheta)}{-\pi x^{2}} \mathrm{~d} x+O\left(\frac{1}{T}\right)
$$

Démonstration. On a

$$
B(\vartheta)=\int_{1-}^{\infty} \frac{\mathrm{d} Z(x, x ; \vartheta)}{-\pi x}=\int_{1}^{\infty} \frac{Z(x, x ; \vartheta)}{-\pi x^{2}} \mathrm{~d} x .
$$

La majoration

$$
Z(x, x ; \vartheta) \ll x^{2}\|\vartheta\| /\left(1+x^{2}\|\vartheta\|^{2}\right) \quad(x \geqslant 2, \vartheta \in \mathbb{R})
$$

implique donc la formule annoncée.

Fin de la démonstration du Théorème 2.2.

Rappelons la notation $Y_{1}$ définie en (6.4). Nous observons d'abord que l'on peut disposer simplement du cas $\|\vartheta\| \leqslant 1 / Y_{1}$. En effet, on a alors $\varrho\left(u_{\vartheta, y}^{*}\right) \ll 1 /(\log y)^{A}$, et il suffit donc de montrer que $B(\vartheta ; y) \ll 1 /(\log y)^{A}$. Or, on déduit immédiatement de $(8 \cdot 2)$ et $(6 \cdot 2)$ que l'on a, pour ces valeurs de $\vartheta$,

$$
B(\vartheta ; y)=-\sum_{P(n) \leqslant y} \frac{\sin (2 \pi n \vartheta)}{\pi n} \ll\|\vartheta\| \Psi\left(\frac{1}{\|\vartheta\|}, y\right)+\sum_{\substack{P(n) \leqslant y \\ n>1 /\|\vartheta\|}} \frac{1}{n} \ll \frac{1}{(\log y)^{A}}
$$


Nous supposons dans toute la suite de cette démonstration que

$$
\|\vartheta\|>1 / Y_{1}
$$

Posons $B=4 A+20, T:=(\log y)^{B}$ et $q_{y}:=q(\vartheta ; y / T)$. Nous procédons différemment selon que $q_{y}=1$ ou $q_{y}>1$.

Lorsque $q_{y}=1$, et donc $\|\vartheta\| \leqslant 2 T / y$ en vertu du théorème de Dirichlet, nous allons voir que la contribution de l'intervalle

$$
I:=\left[\frac{1}{\|\vartheta\| T}, \frac{T}{\|\vartheta\|}\right]
$$

à l'intégrale de (8.1) en fournit une bonne approximation. On déduit en effet du Lemme 6.4 que

$$
\begin{aligned}
Z(x, y ; \vartheta) & -\varrho(u) Z(x, x ; \vartheta) \\
& \ll x \frac{\varrho\left(u_{\vartheta, y}^{*}\right) \log \left(u_{\vartheta, y}^{*}+2\right)}{\log y} G(x\|\vartheta\|)+\frac{x}{(\log y)^{A+1}} \quad(x \in I),
\end{aligned}
$$

où nous avons posé $G(v):=v(|\log v|+1) /\left(1+v^{2}\right)$. Nous notons que $G(v) / v$ est intégrable sur $\mathbb{R}^{+}$.

On a

$$
\varrho(u)-\varrho\left(u_{\vartheta, y}^{*}\right) \ll \frac{\varrho\left(u_{\vartheta, y}^{*}\right) \log \left(u_{\vartheta, y}^{*}+2\right)}{\log y}|\log (x\|\vartheta\|)| \quad(x \in I) .
$$

En utilisant $(8 \cdot 4)$, nous déduisons donc de ce qui précède que

$$
\begin{aligned}
Z(x, y ; \vartheta)-\varrho\left(u_{\vartheta, y}^{*}\right) & Z(x, x ; \vartheta) \\
& \ll x \frac{\varrho\left(u_{\vartheta, y}^{*}\right) \log \left(u_{\vartheta, y}^{*}+2\right)}{\log y} G(\|\vartheta\| x)+\frac{x}{(\log y)^{A+1}} \quad(x \in I) .
\end{aligned}
$$

En reportant cette estimation dans (8·3), nous obtenons

$$
\begin{aligned}
& \int_{I} \frac{Z(x, y ; \vartheta)-Z(x, x ; \vartheta)}{-\pi x^{2}} \mathrm{~d} x \\
& \quad=\left\{\varrho\left(u_{\vartheta, y}^{*}\right)-1\right\} B(\vartheta)+O\left(\frac{\varrho\left(u_{\vartheta, y}^{*}\right) \log \left(u_{\vartheta, y}^{*}+2\right)}{\log y}+\frac{1}{(\log y)^{A}}\right) .
\end{aligned}
$$

Il reste donc à majorer la contribution du complémentaire de l'intervalle $I$ à l'intégrale de (8·3). Pour $x \leqslant 1 /(\|\vartheta\| T)$, la majoration triviale

$$
|Z(x, x ; \vartheta)|+|Z(x, y ; \vartheta)| \ll\|\vartheta\| x^{2}
$$


suffit : on a

$$
\begin{aligned}
\int_{2}^{1 /(\|\vartheta\| T)} \frac{Z(x, y ; \vartheta)-Z(x, x ; \vartheta)}{x^{2}} \mathrm{~d} x & \ll\|\vartheta\| \int_{0}^{1 /(\|\vartheta\| T)} \mathrm{d} x \\
& \ll 1 /(\log y)^{B}
\end{aligned}
$$

Pour $x>T /\|\vartheta\|$, nous traitons séparément les contributions de $Z(x, x ; \vartheta)$ et $Z(x, y ; \vartheta)$. Nous avons d'une part, compte tenu de la majoration triviale $|Z(x, x ; \vartheta)| \ll 1 /\|\vartheta\|$,

$$
\int_{T /\|\vartheta\|}^{\infty} \frac{Z(x, x ; \vartheta)}{x^{2}} \mathrm{~d} x \ll \frac{1}{\|\vartheta\|} \int_{T /\|\vartheta\|}^{\infty} \frac{\mathrm{d} x}{x^{2}} \ll \frac{1}{T} .
$$

D'autre part, en remarquant que l'inégalité $\|\vartheta\|>T / x$ implique

$$
q^{*}:=q(\vartheta ; x / T) \geqslant 2
$$

et donc $\|\vartheta\| \geqslant 1 / 2 q^{*}$ d'où $q^{*} \geqslant 1 / 2\|\vartheta\| \geqslant y / 2(\log y)^{B}$, nous déduisons du Lemme 6.5 que l'on a pour $x \geqslant y$

$$
|Z(x, y ; \vartheta)| \ll x(\log x)^{4} /(\log y)^{B / 4} \quad(x>T /\|\vartheta\|) .
$$

Il s'ensuit que

$$
\int_{T /\|\vartheta\|}^{Y_{1}} \frac{Z(x, y ; \vartheta)}{-\pi x^{2}} \mathrm{~d} x \ll(\log y)^{5-B / 4} \ll(\log y)^{-A} .
$$

Comme le Lemme 8.2 fournit en outre

$$
\left|\int_{Y_{1}}^{\infty} \frac{Z(x, y ; \vartheta)}{-\pi x^{2}} \mathrm{~d} x\right| \leqslant \int_{Y_{1}}^{\infty} \frac{\Psi(x, y)}{x^{2}} \mathrm{~d} x \ll \frac{1}{(\log y)^{A}}
$$

nous obtenons bien

$$
\int_{T /\|\vartheta\|}^{\infty}\{Z(x, y ; \vartheta)-Z(x, x ; \vartheta)\} \frac{\mathrm{d} x}{x^{2}} \ll \frac{1}{(\log y)^{A}} .
$$

Compte tenu de $(8 \cdot 6)$ et $(8 \cdot 8)$, cela établit bien $(2 \cdot 6)$ sous les conditions (8.5) et $q_{y}=1$.

Considérons maintenant l'éventualité $q_{y}=q(\vartheta ; y / T) \geqslant 2$. Notons que cela implique $\|\vartheta\| \gg T / y$ et donc $u_{\vartheta, y}^{*} \leqslant 1$. La contribution de $[2, y]$ à l'intégrale du Lemme 8.1 est nulle et nous avons

$$
\int_{y}^{\infty} \frac{Z(x, x ; \vartheta)}{x^{2}} \mathrm{~d} x \ll \frac{1}{\|\vartheta\|} \int_{y}^{\infty} \frac{\mathrm{d} x}{x^{2}}=\frac{1}{\|\vartheta\| y} \ll \frac{1}{T} .
$$


En reportant dans $(8 \cdot 1)$, nous obtenons donc

$$
\nabla_{\mathbf{1}}(\vartheta ; y)=-\int_{y}^{\infty} \frac{Z(x, y ; \vartheta)}{\pi x^{2}} \mathrm{~d} x+O\left(\frac{1}{T}\right) .
$$

Soit $T_{x}:=(\log x)^{B}$. La définition de $t \mapsto q(\vartheta ; t)$ implique que, pour $x \geqslant y$ et $y$ assez grand, on a soit $q\left(\vartheta ; x / T_{x}\right)=q_{y}=q(\vartheta ; y / T)$, soit $q\left(\vartheta ; x / T_{x}\right)>y / T$. Les Lemmes 6.5 et 6.6 fournissent donc pour $x \geqslant y, q=q_{y}$,

$$
\begin{aligned}
& Z(x, y ; \vartheta) \\
& \ll \frac{x 2^{\omega(q)}(\log q) \varrho(u) \log (u+1)}{\varphi(q) \log y}\left\{\frac{x\left|\vartheta_{q}\right|}{1+x^{2} \vartheta_{q}^{2}}+J_{q}(x, y)\right\}+\frac{x}{(\log x)^{A+1}} .
\end{aligned}
$$

Reportons cette majoration dans (8.11) pour évaluer la contribution à l'intégrale du domaine pour $y \leqslant x \leqslant Y_{1}$ et estimons celle du domaine complémentaire $x>Y_{1}$ par le Lemme 8.2. Nous obtenons

$$
\nabla_{\mathbf{1}}(\vartheta ; y) \ll \frac{2^{\omega(q)}(\log q)^{2}}{\varphi(q) \log y}+\frac{1}{(\log y)^{A}}
$$

avec $q=q_{y} \geqslant 2$. Cette estimation coïncide bien avec (2.7) lorsque $\|\vartheta\| \gg T / y$. Cela termine la preuve du Théorème 2.2 .

\section{Démonstration des critères de $\boldsymbol{\vartheta}$-adaptation}

\subsection{Discrépance de la suite $\{\langle\vartheta n\rangle\}_{n \in S(x, y)}$}

Soit

$$
D(x, y ; \vartheta):=\sup _{0 \leqslant \alpha<\beta \leqslant 1}\left|\sum_{\substack{n \in S(x, y) \\\langle\vartheta \vartheta\rangle \in[\alpha, \beta]}} 1-(\beta-\alpha) \Psi(x, y)\right|
$$

la discrépance de la suite $\{\langle\vartheta n\rangle\}_{n \in S(x, y)}$. Le résultat suivant nous sera utile à plusieurs reprises.

Lemme 9.1. Soit $A>0$. Il existe une constante $B=B_{A}$ telle que, pour $x \geqslant y \geqslant 2, Q:=x /(\log x)^{B}, q:=q(\vartheta ; Q)$, on ait uniformément

$$
D(x, y ; \vartheta) \ll \frac{\Psi(x, y)}{q}+\frac{x}{(\log x)^{A}} .
$$

Démonstration. Compte tenu de la majoration triviale $D(x, y ; \vartheta) \ll \Psi(x, y)$ et du Lemme 6.1 , nous pouvons supposer $y \leqslant x \leqslant Y_{1}:=y^{\log _{2} y}$ et $q \geqslant 2$. L'inégalité d'Erdős-Turán (voir par exemple [20], théorème 2.5) implique, pour tout $H \geqslant 1$,

$$
D(x, y ; \vartheta) \ll \frac{\Psi(x, y)}{H}+\sum_{1 \leqslant h \leqslant H} \frac{|E(x, y ; h \vartheta)|}{h} .
$$


Nous choisissons $H:=\min \left\{q-1,(\log x)^{A}\right\}$. D'après le théorème 10 de [14] et le Lemme 6.5 , on peut choisir $B$ tel que, sous la condition $q:=q(\vartheta ; Q) \geqslant 2$, on ait

$$
|E(x, y ; \vartheta)| \ll \Psi(x, y) \frac{2^{\omega(q)}(\log q) \log (u+1)}{\varphi(q) \log y}+\frac{x}{(\log x)^{A+1}} .
$$

Nous allons appliquer cette majoration pour les valeurs $h \vartheta(1 \leqslant h \leqslant H)$ avec $q(h \vartheta ; 2 Q)$ à la place de $q$. Posons $q_{h}=q /(h, q)$. D'après le Lemme 6.2 , on a (non exclusivement) soit $q(h \vartheta ; 2 Q)=q_{h}$, soit $q(h \vartheta ; 2 Q) \geqslant Q / 2 h$. Cela implique bien $q(h \vartheta ; 2 Q) \geqslant 2$ pour $1 \leqslant h \leqslant H$. En remarquant que

$$
\max _{d \mid q} d 2^{\omega(d)}(\log d) / \varphi(d)=q 2^{\omega(q)}(\log q) / \varphi(q)
$$

nous déduisons de $(9 \cdot 3)$ que

$$
|E(x, y ; h \vartheta)| \ll \frac{q 2^{\omega(q)} \log q}{\varphi(q) q_{h}} \Psi(x, y) \frac{\log (u+1)}{\log y}+\frac{x}{(\log x)^{A+1}} .
$$

Maintenant, nous avons

$$
\begin{aligned}
\sum_{1 \leqslant h \leqslant H} \frac{1}{h q_{h}} & =\frac{1}{q} \sum_{1 \leqslant h \leqslant H} \frac{1}{h} \sum_{d \mid(q, h)} \varphi(d) \\
& \leqslant \frac{1}{q} \sum_{d \mid q} \frac{\varphi(d)}{d} \sum_{1 \leqslant k \leqslant H / d} \frac{1}{k} \ll \frac{\tau(q) \log q}{q} .
\end{aligned}
$$

En reportant $(9 \cdot 4)$ dans $(9 \cdot 2)$ et en tenant compte de l'hypothèse $x \leqslant Y_{1}$, nous obtenons donc

$$
D(x, y ; \vartheta) \ll \Psi(x, y)\left\{\frac{1}{H}+\frac{\tau(q)^{2}(\log q)^{2} \log _{3} y}{\varphi(q) \log y}\right\}+\frac{x}{(\log x)^{A}} .
$$

En observant que le dernier terme domine dès que, par exemple, $q \geqslant(\log x)^{A+1}$, on constate sans peine que cette majoration implique bien $(9 \cdot 1)$.

Le Lemme 9.1 permet une estimation des moments absolus de $\nabla(m \vartheta ; y)$ sur $S(x, y)$ qui, à son tour, sera utilisée dans la preuve du Théorème 3.1.

Lemme 9.2. Soient $A>0, \beta>0, \varepsilon>0$. Il existe une constante $C=C(A, \beta)>0$ telle que, l'on ait uniformément pour $x \geqslant y \geqslant 2, Q_{y}:=y /(\log y)^{C}, \vartheta \in \mathbb{R}$, $q:=q\left(\vartheta ; Q_{y}\right)$,

$$
\sum_{n \in S(x, y)}|\nabla(n \vartheta ; y)|^{\beta} \ll \frac{\Psi(x, y)}{q^{(1-\varepsilon) \beta_{1}(\log y)^{\beta}}}+\frac{x}{(\log x)^{A}},
$$

avec $\beta_{1}:=\min (1, \beta / 2)$. 
Démonstration. Au vu de la majoration $\sup _{\vartheta \in \mathbb{R}}|\nabla(\vartheta ; y)| \ll 1 / \log y$, issue du Théorème 2.1 , et de la majoration $6 \cdot 2$ pour $\Psi(x, y)$, nous pouvons supposer $y \leqslant x \leqslant Y_{1}$.

Soit $C:=\max \left\{5 A+30, B_{3 A+2}\right\}$ où $A \mapsto B_{A}$ est la fonction apparaissant au Lemme 9.1. Nous posons $Q_{y}:=y /(\log y)^{C}, q:=q\left(\vartheta ; Q_{y}\right)$, et nous introduisons un paramètre $M$ satisfaisant à $1 \leqslant M \leqslant(\log y)^{O(1)}$. Pour $1 \leqslant m \leqslant M$, nous posons

$$
E_{m}:=\left\{\vartheta_{1} \in \mathbb{R}: q\left(\vartheta_{1} ; Q_{y}\right)=m\right\}, \quad E_{M}^{*}:=\mathbb{R} \backslash \cup_{1 \leqslant m \leqslant M} E_{m} .
$$

D'après le Théorème 2.1, on a

$$
\sup _{\vartheta_{1} \in E_{m}}\left|\nabla\left(\vartheta_{1} ; y\right)\right| \ll \frac{1}{m^{1-\varepsilon} \log y}, \quad \sup _{\vartheta_{1} \in E_{M}^{*}}\left|\nabla\left(\vartheta_{1} ; y\right)\right| \ll \frac{1}{M^{1-\varepsilon} \log y}
$$

Nous remarquons ensuite que

$$
\vartheta_{1} \in E_{m} \Rightarrow\left\langle\vartheta_{1}\right\rangle \in \bigcup_{\substack{1 \leqslant a \leqslant m \\(a, m)=1}}\left[\frac{a}{m}-\frac{1}{m Q_{y}}, \frac{a}{m}+\frac{1}{m Q_{y}}[,\right.
$$

et l'ensemble figurant au membre de droite peut être écrit comme une réunion d'au plus $\varphi(m)+1$ intervalles du tore $\mathbb{T}=\mathbb{R} / \mathbb{Z}$, d'une mesure totale n'excédant pas $2 / Q_{y} \leqslant 2 / q$. Il résulte donc du Lemme 9.1 que

$$
\sum_{\substack{n \in S(x, y) \\ n \vartheta \in E_{m}}} 1 \ll \frac{m \Psi(x, y)}{q}+\frac{x}{(\log x)^{2 A+1}} \quad(1 \leqslant m \leqslant M) .
$$

Nous déduisons de cette majoration et de (9.6) que

$$
\begin{aligned}
\sum_{n \in S(x, y)}|\nabla(n \vartheta ; y)|^{\beta} & \leqslant \sum_{1 \leqslant m \leqslant M} \sup _{\vartheta_{1} \in E_{m}}\left|\nabla\left(\vartheta_{1} ; y\right)\right|^{\beta} \sum_{\substack{n \in S(x, y) \\
n \vartheta \in E_{m}}} 1+\sum_{\substack{n \in S(x, y) \\
n \vartheta \in E_{M}^{*}}} \sup _{\vartheta_{1} \in E_{M}^{*}} \mid \nabla\left(\vartheta_{1} ; y\right)^{\beta} \\
& \ll \sum_{1 \leqslant m \leqslant M}\left\{\frac{m^{1-\beta(1-\varepsilon)} \Psi(x, y)}{q(\log y)^{\beta}}+\frac{x}{(\log x)^{2 A+1}}\right\}+\frac{\Psi(x, y)}{M^{(1-\varepsilon) \beta(\log y)^{\beta}}} \\
& \ll \frac{\Psi(x, y)}{(\log y)^{\beta}}\left\{\frac{M^{2-2 \beta_{1}+2 \beta \varepsilon}}{q}+\frac{1}{M^{\beta(1-\varepsilon)}}\right\}+\frac{x}{(\log x)^{A}} .
\end{aligned}
$$

La majoration requise (9.5) en découle en choisissant $M^{\beta}=\min \left\{q^{\beta_{1}},(\log y)^{A+1}\right\}$, quitte à altérer la valeur de $\varepsilon$. 


\subsection{Critère de $\vartheta$-adaptation lorsque $f \in \mathcal{L}^{\alpha}\left(\mathbb{N}^{*}\right)$ : preuve du}

Théorème 3.1

Le Lemme 9.2 contient, sur le comportement en moyenne de $|\nabla(n \vartheta ; y)|$, une information suffisante pour nous permettre de prouver le Théorème 3.1 . D'après $(1 \cdot 22)$, on a

$$
\nabla_{f}(\vartheta ; y)=\sum_{P(n) \leqslant y} \frac{f(n)}{n} \nabla(n \vartheta ; y)
$$

et il s'agit ici d'établir, sous l'hypothèse $f \in \mathcal{L}^{\alpha}\left(\mathbb{N}^{*}\right)$, que cette quantité tend vers 0 quand $y$ tend vers l'infini. Nous fournissons en fait une forme quantitative de cette assertion.

Lemme 9.3. Soient $\varepsilon>0, \alpha>1, f \in \mathcal{L}^{\alpha}$. Il existe une constante $C>0$ telle que l'on ait, uniformément pour $y \geqslant 2, Q_{y}:=y /(\log y)^{C}, \vartheta \in \mathbb{R}, q:=q\left(\vartheta ; Q_{y}\right)$,

$$
\nabla_{f}(\vartheta ; y) \ll \frac{1}{q^{(1-\varepsilon) \beta_{1} / \beta}}+\frac{\log _{2} y}{\log y}
$$

avec $1 / \beta=1-1 / \alpha, \beta_{1}:=\min (1, \beta / 2)$.

Démonstration. Le cas $\vartheta \in \mathbb{Q}$ résulte aisément de $(2 \cdot 4)$. En effet, l'hypothèse $f \in \mathcal{L}^{\alpha}$ implique trivialement, via l'inégalité de Hölder et $(6 \cdot 2)$,

$$
\sum_{n \in S(x, y)}|f(n)| \ll\left\{\sum_{n \leqslant x}|f(n)|^{\alpha}\right\}^{1 / \alpha} \Psi(x, y)^{1 / \beta} \ll x \mathrm{e}^{-u / 2 \beta}
$$

d'où, par sommation d'Abel,

$$
\sum_{P(n) \leqslant y} \frac{|f(n)|}{n} \ll \log y .
$$

Il s'ensuit donc, en vertu de $(2 \cdot 4)$, que l'on a pour chaque $A>0$ fixé et uniformément par rapport à $y \geqslant 2, a \in \mathbb{Z}, q \leqslant y /(\log y)^{5 A+25}$

$$
\sum_{P(n) \leqslant y} \frac{f(n)}{n} \nabla(n a / q ; y) \ll \frac{1}{(\log y)^{A}} .
$$

Choisissons $A=1$. Cela fournit l'assertion de l'énoncé dans le cas $\vartheta \in \mathbb{Q}$, puisque la valeur $C=30$ convient.

Supposons dorénavant $\vartheta \in \mathbb{R} \backslash \mathbb{Q}$ et posons, avec les notations du Lemme 9.2, $C:=C(2 \beta, \beta)+30, Q_{y}:=y /(\log y)^{C}, q=q\left(\vartheta ; Q_{y}\right)$, de sorte que $q$ tend vers l'infini avec $y$. L'inégalité de Hölder et (9.5) impliquent

$$
\begin{aligned}
\sum_{n \in S(x, y)} f(n) \nabla(n \vartheta ; y) & \ll\left\{\sum_{n \leqslant x}|f(n)|^{\alpha}\right\}^{1 / \alpha}\left\{\sum_{n \in S(x, y)}|\nabla(n \vartheta ; y)|^{\beta}\right\}^{1 / \beta} \\
& \ll \frac{x \mathrm{e}^{-u / 2 \beta}}{q^{(1-\varepsilon) \beta_{1} / \beta} \log y}+\frac{x}{(\log x)^{2}} .
\end{aligned}
$$


Par sommation d'Abel, il suit

$$
\sum_{\substack{P(n) \leqslant y \\ n>y}} \frac{f(n)}{n} \nabla(n \vartheta ; y) \ll \frac{1}{q^{(1-\varepsilon) \beta_{1} / \beta}}+\frac{1}{\log y} .
$$

Il reste à majorer

$$
\sum_{n \leqslant y} \frac{f(n)}{n} \nabla(n \vartheta ; y)
$$

À cette fin, nous utilisons l'estimation $(2 \cdot 3)$ du Théorème 2.1 pour majorer $\nabla(n \vartheta ; y)$ pour tous les entiers $n \leqslant y$ sauf peut-être ceux d'un sous-ensemble dont la contribution globale à $(9 \cdot 12)$ est trivialement acceptable. Comme $C>30$, on a $2 Q_{y} \leqslant y /(\log y)^{30}$ pour $y$ assez grand. Or, d'après le Lemme 6.2, pour $n \leqslant Q_{y} /(\log y)^{2}$, on a $q\left(n \vartheta ; 2 Q_{y}\right)=q /(n, q)$ ou $q\left(n \vartheta ; 2 Q_{y}\right) \geqslant \frac{1}{2}(\log y)^{2}$. Le Théorème 2.1 implique donc

$$
\begin{gathered}
\sum_{n \leqslant Q_{y} /(\log y)^{2}} \frac{f(n)}{n}|\nabla(n \vartheta ; y)| \ll \frac{1}{\log y} \sum_{n \leqslant y} \frac{|f(n)|}{n}\left\{\sqrt{\frac{(q, n)}{q}}+\frac{1}{\log y}\right\} \\
\ll \frac{1}{(\log y) \sqrt{q}} \sum_{d \mid q} \sqrt{d} \sum_{\substack{n \leqslant y \\
n \equiv 0(\bmod d)}} \frac{|f(n)|}{n}+\frac{1}{\log y}
\end{gathered}
$$

où nous avons fait appel à (9-10). Appliquant une nouvelle fois l'inégalité de Hölder, on peut écrire, grâce à l'hypothèse $f \in \mathcal{L}^{\alpha}$,

$$
\sum_{\substack{n \leqslant y \\ n \equiv 0(\bmod d)}} \frac{|f(n)|}{n} \ll\left\{\sum_{\substack{n \leqslant y \\ n \equiv 0(\bmod d)}} \frac{1}{n}\right\}^{1 / \beta}\left\{\sum_{n \leqslant y} \frac{|f(n)|^{\alpha}}{n}\right\}^{1 / \alpha} \ll \frac{\log y}{d^{1 / \beta}} .
$$

Reportons dans (9.13) en tenant compte de l'inégalité

$$
\sum_{d \mid q} d^{1 / 2-1 / \beta} \leqslant \tau(q) q^{1 / 2-\beta_{1} / \beta} \quad(\beta>1, q \geqslant 1) .
$$

Nous obtenons

$$
\sum_{n \leqslant Q_{y} /(\log y)^{2}} \frac{|f(n)|}{n}|\nabla(n \vartheta ; y)| \ll \frac{\tau(q)}{q^{\beta_{1} / \beta}}+\frac{1}{\log y} .
$$

La majoration $\nabla(n \vartheta ; y) \ll 1 / \log y$ issue du Théorème 2.1 fournit enfin

$$
\begin{aligned}
\sum_{Q_{y} /(\log y)^{2}<n \leqslant y} \frac{|f(n)|}{n}|\nabla(n \vartheta ; y)| & \ll \frac{1}{\log y} \sum_{Q_{y} /(\log y)^{2}<n \leqslant y} \frac{|f(n)|}{n} \\
& \ll \frac{\log _{2} y}{\log y},
\end{aligned}
$$

où nous avons estimé la dernière somme en $n$ par sommation d'Abel à partir de $(9 \cdot 9)$.

La conjonction des relations $(9 \cdot 11),(9 \cdot 14)$ et $(9 \cdot 15)$ implique bien $(9 \cdot 8)$. 


\section{3. Preuve du Théorème 3.2}

Le fait que l'hypothèse $(3 \cdot 1)$ est une condition suffisante de $\vartheta$-adaptation résulte trivialement du Théorème 2.1 et de l'expression (9.7). La preuve de la seconde assertion de l'énoncé, concernant l'optimalité de cette condition, repose sur le lemme suivant. Nous conservons la notation $Y_{1}$ de (6.4).

Lemme 9.4. On a, uniformément pour $y \geqslant 2$ et $0<\|\vartheta\| \leqslant 1 / Y_{1}$,

$$
\nabla(\vartheta ; y)=\frac{\mathrm{e}^{-\gamma}}{\log y}\left\{\frac{1}{2} \operatorname{sgn} B(\vartheta)+O\left(\frac{1}{\log y}\right)\right\} .
$$

Démonstration. Les deux membres de la formule à établir sont des fonctions 1périodiques impaires. Nous pouvons donc supposer sans perte de généralité que $0<\vartheta \leqslant 1 / Y_{1}$. Nous avons

$$
\nabla(\vartheta ; y)=\sum_{n \in S(1 / \vartheta, y)} \frac{\mu(n)}{n}\left\{n \vartheta-\frac{1}{2}\right\}+O\left(\sum_{\substack{P(n) \leqslant y \\ n>1 / \vartheta}} \frac{1}{n}\right) .
$$

La majoration (6.2) et le Lemme 8.2 fournissent

$$
\begin{aligned}
\nabla(\vartheta ; y) & =-\frac{1}{2} \sum_{P(n) \leqslant y} \frac{\mu(n)}{n}+O\left(\vartheta \Psi(1 / \vartheta, y)+\frac{1}{(\log y)^{2}}\right) \\
& =-\frac{1}{2} \prod_{p \leqslant y}\left(1-\frac{1}{p}\right)+O\left(\frac{1}{(\log y)^{2}}\right) .
\end{aligned}
$$

On conclut en appliquant la formule de Mertens — voir, e.g., [29], théorème I.1.11.

Nous sommes maintenant en mesure d'établir la seconde assertion du Théorème 3.2. Nous construisons à cette fin, par une méthode analogue à celle de Liouville, des nombres réels $\vartheta$ ayant des propriétés d'approximation adéquates. Étant donnée une suite croissante de nombres premiers $\left\{p_{r}\right\}_{r \geqslant 1}$ satisfaisant

$$
\mathrm{e}^{r^{4 r}} \leqslant p_{r}<2 \mathrm{e}^{r^{4 r}},
$$

on définit $\vartheta$ par

$$
\vartheta:=\sum_{r \geqslant 1} \frac{1}{p_{r}} .
$$

Les approximations rationnelles définies par

$$
\frac{a_{m}}{b_{m}}:=\sum_{1 \leqslant r \leqslant m} \frac{1}{p_{r}}, \quad\left(a_{m}, b_{m}\right)=1,
$$


satisfont alors

$$
b_{m}=\prod_{1 \leqslant r \leqslant m} p_{r} \quad(m \geqslant 1) .
$$

On vérifie aisément que pour $m \geqslant 2$ on a

$$
\mathrm{e}^{m^{4 m}} \leqslant b_{m}=\prod_{1 \leqslant r \leqslant m} p_{r} \leqslant \mathrm{e}^{2 m^{4 m}} \leqslant p_{m}^{2}, \quad p_{m+1} \geqslant b_{m}^{2}+2
$$

et

$$
\left|\vartheta-\frac{a_{m}}{b_{m}}\right| \leqslant \frac{2}{p_{m+1}} \leqslant \frac{\mathrm{e}^{-m^{4 m+2}}}{b_{m}} .
$$

Nous définissons ensuite une fonction arithmétique $f$ par

$$
f(n):= \begin{cases}b_{m} & \text { si } b_{m} \leqslant n \leqslant b_{m}^{2}, b_{m} \mid n \\ 0 & \text { sinon. }\end{cases}
$$

La fonction $f$ satisfait la condition $(3 \cdot 2)$. En effet,

$$
\sum_{P(n) \leqslant y} \frac{f(n)}{n}=\sum_{P\left(b_{m}\right) \leqslant y} \sum_{\substack{b_{m}<n \leqslant b_{m}^{2} \\ b_{m} \mid n}} \frac{b_{m}}{n} \ll \sum_{P\left(b_{m}\right) \leqslant y} \log b_{m} \ll \sum_{p_{m} \leqslant y} \log p_{m} \ll \log y .
$$

Nous allons prouver que $\lim \sup _{y \rightarrow \infty}\left|\nabla_{f}(\vartheta ; y)\right| \geqslant \frac{1}{2} \mathrm{e}^{-\gamma}$, et plus précisément

$$
\lim _{m \rightarrow \infty}\left|\nabla_{f}\left(\vartheta ; b_{m}\right)\right|=\frac{1}{2} \mathrm{e}^{-\gamma}
$$

ce qui montrera bien que $(f, g)$ n'est pas $\vartheta$-adapté.

Nous pouvons écrire

$$
\nabla_{f}\left(\vartheta ; b_{m}\right)=\sum_{1 \leqslant k \leqslant m} \delta_{k, m}
$$

avec

$$
\delta_{k, m}:=\sum_{b_{k}<n \leqslant b_{k}^{2}} \frac{f(n)}{n} \nabla\left(n \vartheta ; b_{m}\right)=\sum_{1<\ell \leqslant b_{k}} \frac{\nabla\left(\ell b_{k} \vartheta ; b_{m}\right)}{\ell} \ll \frac{k^{4 k}}{m^{4 m}},
$$

en vertu de la majoration $\nabla\left(n \vartheta ; b_{m}\right) \ll 1 / \log b_{m} \ll 1 / m^{4 m}$ issue du Théorème 2.1 . Il vient

$$
\sum_{k<m} \delta_{k, m} \ll \frac{1}{m}=o(1)
$$

Il reste donc seulement à estimer $\delta_{m, m}$. Pour $m$ assez grand et $n=\ell b_{m} \leqslant b_{m}^{2}$, on a $\|n \vartheta\| \leqslant b_{m}\left\|b_{m} \vartheta\right\| \leqslant b_{m}^{-\log _{2} b_{m}}$ et $\operatorname{sgn} B(n \vartheta)=\operatorname{sgn} B\left(b_{m} \vartheta\right)$. Les Lemmes 9.4 et 8.2 fournissent donc

$$
\begin{aligned}
\delta_{m, m} & =\sum_{1<\ell \leqslant b_{m}} \frac{\mathrm{e}^{-\gamma}}{\ell \log b_{m}}\left\{\frac{1}{2} \operatorname{sgn} B\left(b_{m} \vartheta\right)+O\left(\frac{1}{\log b_{m}}\right)\right\} \\
& =\frac{1}{2} \mathrm{e}^{-\gamma} \operatorname{sgn} B\left(b_{m} \vartheta\right)+O\left(\frac{1}{\log b_{m}}\right) .
\end{aligned}
$$

En reportant $(9 \cdot 23)$ et $(9 \cdot 24)$ dans $(9 \cdot 22)$, nous obtenons bien $(9 \cdot 21)$. 


\subsection{Critère de $\vartheta$-adaptation lorsque $\vartheta \in \mathbb{Q}:$ preuve du Théo- rème 3.4}

On considère le cas $\vartheta=a / q$ où $(a, q)=1$ et $q$ est fixé. La majoration $(2 \cdot 4)$ implique $\nabla(n \vartheta ; y) \ll 1 /(\log y)^{A+1}$ pour tout $n \in \mathbb{N}$. On en déduit grâce à $(1 \cdot 22)$ que $\nabla_{f}(\vartheta ; y)=o(1)$ pour toute fonction $f$ satisfaisant à

$$
\sum_{P(n) \leqslant y} \frac{|f(n)|}{n} \ll(\log y)^{A}
$$

où $A$ est une constante arbitrairement fixée. Étant donné que

$$
\sum_{P(n) \leqslant y} \frac{|f(n)|}{n} \leqslant \sum_{P(n) \leqslant y} \frac{|g(n)|}{n} \sum_{P(n) \leqslant y} \frac{1}{n} \ll(\log y) \sum_{P(n) \leqslant y} \frac{|g(n)|}{n},
$$

il est clair que la condition

$$
\sum_{P(n) \leqslant y} \frac{|g(n)|}{n} \ll(\log y)^{A}
$$

est également suffisante.

\subsection{Critère de $\vartheta$-adaptation lorsque $g \in \mathcal{L}^{\alpha}\left(\mathbb{N}^{*}\right)$ : preuve du}

Théorème 3.5

Nous établissons d'abord un critère de $\vartheta$-adaptation directement issu du Théorème 2.2 .

Lemme 9.5. Soient $\alpha>1, \vartheta \in \mathbb{R}$ et $(f, g)$ un couple de fonctions arithmétiques satisfaisant (1.3) et $g \in \mathcal{L}^{\alpha}$. Alors $(f, g)$ est $\vartheta$-adapté si, et seulement si,

$$
\lim _{y \rightarrow \infty} \sum_{P(n) \leqslant y} g(n)\left\{1-\varrho\left(u_{n \vartheta, y}^{*}\right)\right\} \frac{B(n \vartheta)}{n}=0 .
$$

Démonstration. D'après $(1 \cdot 22)$ et $(1 \cdot 20)$, on a

$$
\nabla_{f}(\vartheta ; y)=\sum_{P(n) \leqslant y} \frac{g(n)}{n}\{B(n \vartheta)-B(n \vartheta ; y)\}
$$

Or, la formule $(2 \cdot 6)$ permet d'approcher $B(n \vartheta)-B(n \vartheta ; y)$ par $\left\{1-\varrho\left(u_{n \vartheta, y}^{*}\right)\right\} B(n \vartheta)$ avec une erreur qui est, à un facteur $(\log q)^{2}$ près, de l'ordre du majorant fourni par $(2 \cdot 3)$ pour $\nabla(n \vartheta ; y)$. La moyenne pondérée de ces erreurs peut donc être évaluée, sous l'hypothèse $g \in \mathcal{L}^{\alpha}$, avec des calculs identiques à ceux de la preuve du Théorème 3.1 pour la majorer $\nabla_{f}(\vartheta ; y)$ sous l'hypothèse $f \in \mathcal{L}^{\alpha}$. Nous obtenons ainsi que cette moyenne est $o(1)$, soit

$$
\nabla_{f}(\vartheta ; y)=\sum_{P(n) \leqslant y} \frac{g(n)}{n} B(n \vartheta)\left\{1-\varrho\left(u_{n \vartheta, y}^{*}\right)\right\}+o(1) \quad(y \rightarrow \infty)
$$

Cela implique bien le résultat requis. 
Fin de la démonstration du Théorème 3.5.

Commençons par établir que la condition (a) est suffisante pour la $\vartheta$-adaptation. On a $\varrho\left(u_{\vartheta, y}^{*}\right)=1$ dès que $\|\vartheta\|>1 / y$. Lorsque $g$ est bornée, la condition $(9 \cdot 26)$ est donc réalisée dès que

$$
\lim _{y \rightarrow \infty} \sum_{\substack{P(n) \leqslant y \\\|n \vartheta\| \leqslant 1 / y}} \frac{1}{n}=0
$$

Soit $A>0$. D'après le Lemme 9.1, il existe une constante $B=B(A)>0$ telle que, si l'on désigne par $m=m(y)$ l'unique entier tel que $q_{m} \leqslant Q_{y}:=y /(\log y)^{B}<q_{m+1}$, on ait

$$
\sum_{\substack{n \in S(x, y) \\\|n \vartheta\| \leqslant 1 / y}} 1 \ll \frac{\Psi(x, y)}{q_{m}}+\frac{x}{(\log x)^{A}} \quad(x \geqslant y \geqslant 2) .
$$

Comme $q_{m} \leqslant y$, il résulte en outre du Lemme 6.3 que l'inégalité $\|n \vartheta\| \leqslant 1 / y$ a lieu pour au plus six valeurs de $n$ dans chaque intervalle $\left[t q_{m},(t+1) q_{m}[(t \geqslant 1)\right.$. Il s'ensuit que

$$
\sum_{\substack{n \leqslant x \\\|n \vartheta\| \leqslant 1 / y}} 1 \ll \frac{x}{q_{m}} \quad(1 \leqslant x \leqslant y) .
$$

Par sommation d'Abel, on déduit des deux dernières estimations que

$$
\sum_{\substack{P(n) \leqslant y \\\|n \vartheta\| \leqslant 1 / y}} \frac{1}{n} \ll \frac{\log y}{q_{m}}+\frac{1}{(\log y)^{A}} \ll \frac{\log q_{m+1}}{q_{m}}+\frac{1}{(\log y)^{A}}
$$

Cela montre bien que la condition (a) du Théorème 3.5 implique (9·28).

Montrons maintenant que la propriété (b) est également une condition suffisante de $\vartheta$-adaptation. Nous observons d'abord que l'hypothèse $g \in \mathcal{L}^{\alpha}$ implique, pour tout $s, 1<s<\alpha$,

$$
\sum_{P(n) \leqslant y} \frac{|g(n)|^{s}}{n} \ll \log y
$$

Cela se déduit par sommation d'Abel de la majoration

$$
\begin{aligned}
\sum_{n \in S(x, y)}|g(n)|^{s} & \ll \Psi(x, y)^{1-s / \alpha}\left\{\sum_{n \leqslant x}|g(n)|^{\alpha}\right\}^{s / \alpha} \quad(x \geqslant 2, y \geqslant 2) \\
& \ll x \mathrm{e}^{-\frac{1}{2}(1-s / \alpha) u}
\end{aligned}
$$


obtenue par l'inégalité de Hölder et (6·2). Nous déduisons de (9·30) que l'on a, pour $1<s<\alpha, 1 / s+1 / r=1, A>0$,

$$
\begin{aligned}
\left|\sum_{P(n) \leqslant y} g(n)\left\{1-\varrho\left(u_{n \vartheta, y}^{*}\right)\right\} \frac{B(n \vartheta)}{n}\right| & \leqslant\left\{\sum_{P(n) \leqslant y} \frac{|g(n)|^{s}}{n}\right\}^{1 / s}\left\{\sum_{\substack{P(n) \leqslant y \\
\|n \vartheta\| \leqslant 1 / y}} \frac{1}{n}\right\}^{1 / r} . \\
& \ll \frac{\left(\log q_{m+1}\right)^{1 / r}}{q_{m}}+(\log y)^{1-(A+1) / r}
\end{aligned}
$$

d'après (9.29). En choisissant $r$ assez proche de $\alpha /(\alpha-1)$ et $A \geqslant r$, on obtient que cette quantité est $o(1)$. Cela montre bien que la propriété (b) implique la $\vartheta$ adaptation.

Montrons maintenant que le couple $(\tau, \mathbf{1})$ n'est pas $\vartheta$-adapté si

$$
\limsup _{m \rightarrow \infty} \frac{\log q_{m+1}}{q_{m}}>0 .
$$

Soit $A>2$. Il existe une constante positive $c$ et une infinité d'entiers $m$ tels que

$$
q_{m+1}>\mathrm{e}^{c q_{m}}>q_{m}^{8 A} .
$$

Posons $y_{m}:=q_{m+1}^{1 / 2 A}$. Nous allons montrer que, pour un choix convenable de $A$, on a

$$
\left|\nabla_{\tau}\left(\vartheta ; y_{m}\right)\right| \gg 1
$$

lorsque $m$ tend vers l'infini sous la condition (9·32). D'après $(9 \cdot 27)$, on a

$$
\nabla_{\tau}\left(\vartheta ; y_{m}\right)=\sum_{\substack{P(n) \leqslant y_{m} \\\|n \vartheta\| \leqslant 1 / y_{m}}} \frac{B(n \vartheta)}{n}\left\{1-\varrho\left(u_{n \vartheta, y_{m}}^{*}\right)\right\}+o(1) \quad(m \rightarrow \infty)
$$

Décomposons la somme en $n$ du membre de droite sous la forme $D_{1}+D_{2}+D_{3}$, selon les conditions supplémentaires

$$
\begin{array}{ll}
\left(D_{1}\right) & n \leqslant q_{m} \sqrt{q_{m+1}}, q_{m} \nmid n \\
\left(D_{2}\right) & n \leqslant q_{m} \sqrt{q_{m+1}}, q_{m} \mid n \\
\left(D_{3}\right) & n>q_{m} \sqrt{q_{m+1}} .
\end{array}
$$

Nous supposons pour fixer les idées que $B\left(q_{m} \vartheta\right)>0$. Si $n$ satisfait $\left(D_{1}\right)$, on a

$$
\|n \vartheta\| \geqslant \frac{1}{q_{m}}-\frac{n}{q_{m} q_{m+1}} \geqslant \frac{1}{q_{m}}-\frac{1}{\sqrt{q_{m+1}}}>\frac{1}{y_{m}},
$$


donc $D_{1}=0$. Si $n$ satisfait $\left(D_{2}\right)$, on a

$$
\|n \vartheta\| \leqslant n\left\|q_{m} \vartheta\right\| / q_{m} \leqslant n / q_{m} q_{m+1} \leqslant 1 / \sqrt{q_{m+1}}
$$

et donc $B(n \vartheta)=\frac{1}{2}+o(1)$ et $u_{n \vartheta, y_{m}}^{*} \geqslant A$. Il s'ensuit, en décomposant les entiers $n$ de $D_{2}$ sous la forme $n=r q_{m}$, que

$$
\begin{aligned}
D_{2} & \geqslant \frac{1}{2}\{1-\varrho(A)+o(1)\} \sum_{\substack{P(r) \leqslant y_{m} \\
r \leqslant \sqrt{q_{m}+1}}} \frac{1}{r q_{m}} \\
& =\{1-\varrho(A)+o(1)\} \frac{\log q_{m+1}}{4 A q_{m}} \int_{0}^{A} \varrho(v) \mathrm{d} v \geqslant \frac{\log q_{m+1}}{4 A q_{m}},
\end{aligned}
$$

pour $m$ assez grand : la somme en $r$ a été estimée par sommation d'Abel en faisant appel à des estimations standard de $\Psi(x, y)$ et l'on a utilisé l'inégalité

$$
\{1-\varrho(A)\} \int_{0}^{A} \varrho(v) \mathrm{d} v \geqslant\{1-\varrho(2)\} \int_{0}^{2} \varrho(v) \mathrm{d} v>1
$$

Nous majorons $D_{3}$ en utilisant le Lemme 9.1, qui fournit

$$
\sum_{\substack{n \in S\left(x, y_{m}\right) \\\|n \vartheta\| \leqslant 1 / y_{m}}} \ll \frac{\Psi(x, y)}{q_{m}}+\frac{x}{(\log x)^{A}} \quad\left(x \geqslant y_{m}\right) .
$$

Par sommation d'Abel et compte tenu de (9·32), nous déduisons de cette majoration que

$$
D_{3} \ll \frac{\log y_{m}}{q_{m}} \int_{A}^{\infty} \varrho(v) \mathrm{d} v+\frac{1}{\left(\log y_{m}\right)^{A-1}} \ll \frac{\log q_{m+1}}{A q_{m}} \int_{A}^{\infty} \varrho(v) \mathrm{d} v .
$$

Pour un choix convenable de $A$, nous déduisons donc de ce qui précède que l'on a, lorsque $m$ tend vers l'infini sous la condition $(9 \cdot 32)$,

$$
\nabla_{\tau}\left(\vartheta ; y_{m}\right) \gg \frac{\log q_{m+1}}{q_{m}}+o(1)
$$

Cela établit bien $(9 \cdot 33)$. 


\section{Preuve des Théorèmes 4.2 et 4.3}

\subsection{Preuve du Théorème 4.2}

Nous pouvons supposer sans perte de généralité que la fonction $\xi$ est croissante et satisfait

$$
\xi(y)=o(\log y) \quad(y \rightarrow \infty) .
$$

Nous construisons une fonction $g$ et un nombre réel $\vartheta$ conformes aux conditions de l'énoncé à partir d'une suite de nombres premiers $\left\{p_{r}\right\}_{r \geqslant 1}$ astreinte à la condition de croissance $(9 \cdot 16)$. Définissons $\vartheta$ par $(9 \cdot 17)$. Pour définir $g$, nous nous donnons une fonction positive strictement croissante $\xi_{1}$ tendant vers l'infini avec $y$ assez lentement pour que

$$
\xi_{1}(y) \leqslant y \quad(y \geqslant 1), \quad \sum_{k \leqslant r}\left(\sum_{\ell \leqslant \xi_{1}\left(p_{k}\right)} \frac{1}{\ell}\right) \leqslant \xi\left(p_{r}\right) \quad(r \geqslant 1)
$$

Les approximations rationnelles $a_{m} / b_{m}$ de $\vartheta$ étant données par (9·18), nous posons

$$
g(n):= \begin{cases}b_{m} \operatorname{sgn} B(n \vartheta), & \text { si } b_{m} \leqslant n \leqslant b_{m} \xi_{1}\left(p_{m}\right) \text { et } b_{m} \mid n, \\ 0, & \text { sinon. }\end{cases}
$$

Comme $g(n) / n$ ne tend pas vers 0 , la série $V(g ; \vartheta)$ est divergente et $P$-divergente. Montrons qu'on a également également

$$
\sum_{P(n) \leqslant y} \frac{|g(n)|}{n} \leqslant \xi(y) \quad(y \geqslant 1) .
$$

Il suffit de considérer le cas $y>p_{1}$. Soit alors $r \in \mathbb{N}^{*}$ tel que $p_{r}<y \leqslant p_{r+1}$. On a

$$
\sum_{P(n) \leqslant y} \frac{|g(n)|}{n} \leqslant \sum_{1 \leqslant k \leqslant r} \sum_{\ell \leqslant \xi_{1}\left(p_{k}\right)} \frac{b_{k}}{b_{k} \ell} \leqslant \xi\left(p_{r}\right) \leqslant \xi(y) .
$$

Pour établir que $(f, g)$ n'est pas $\vartheta$-adapté nous minorons $\nabla_{f}\left(\vartheta ; b_{m}\right)$. Compte tenu de $(10 \cdot 1),(10 \cdot 2)$ et $(1 \cdot 22)$, le Théorème 2.2 implique

$$
\begin{aligned}
\nabla_{f}\left(\vartheta ; b_{m}\right) & =\sum_{P(n) \leqslant b_{m}} \frac{g(n)}{n}\left\{1-\varrho\left(u_{n \vartheta, y}^{*}\right)\right\} B(n \vartheta)+o(1) \\
& =\sum_{1<\ell \leqslant \xi_{1}\left(b_{m}\right)} \frac{1-\varrho\left(u_{\ell b_{m} \vartheta, y}^{*}\right)}{\ell}\left|B\left(\ell b_{m} \vartheta\right)\right|+o(1)
\end{aligned}
$$

Or, pour $1 \leqslant \ell \leqslant \xi_{1}\left(b_{m}\right)$, nous avons, grâce à $(9 \cdot 20)$ et à l'inégalité $\xi_{1}\left(b_{m}\right) \leqslant b_{m}$,

$$
\left\|\ell b_{m} \vartheta\right\| \leqslant \xi_{1}\left(b_{m}\right) \mathrm{e}^{-m^{4 m+2}} \leqslant b_{m}^{1-m^{2} / 2},
$$


où la dernière inégalité résulte de $(9 \cdot 19)$. Cela implique d'une part

$$
\left|B\left(\ell b_{m} \vartheta\right)\right|=\frac{1}{2}-\left\|\ell b_{m} \vartheta\right\|=\frac{1}{2}+o(1),
$$

et d'autre part, en vertu de la décroissance de la fonction $\varrho$,

$$
1-\varrho\left(u_{\ell b_{m} \vartheta, y}^{*}\right) \geqslant 1-\varrho\left(\frac{1}{2} m^{2}-1\right)=1+o(1) .
$$

En reportant dans (10.3), il vient

$$
\nabla_{f}\left(\vartheta, b_{m}\right) \geqslant\left\{\frac{1}{2}+o(1)\right\} \log \xi_{1}\left(b_{m}\right)
$$

et, par conséquent, $\limsup _{y \rightarrow \infty} \nabla_{f}(\vartheta, y)=\infty$. Cela prouve que le couple $(f, g)$ n'est pas $\vartheta$-adapté.

\section{2. ( $\left.D_{\vartheta}\right)$ pour $(f, g)=(\log , \Lambda)$ : preuve du Théorème 4.3}

Lemme 10.1. Pour tout $\vartheta \in \mathbb{R}$, le couple $(\log , \Lambda)$ est $\vartheta$-adapté, autrement dit

$$
\nabla_{\log }(\vartheta ; y)=\sum_{P(n) \leqslant y} \frac{\Lambda(n)}{n} \nabla_{\mathbf{1}}(n \vartheta ; y)=o(1) \quad(y \rightarrow \infty) .
$$

Démonstration. Le cas $\vartheta \in \mathbb{Q}$ relève du Théorème 3.4. Nous supposons donc dans la suite que $\vartheta$ est irrationnel.

Posons

$$
Q_{y}:=y /(\log y)^{30}, \quad q:=q\left(\vartheta ; Q_{y}\right) .
$$

Comme $\vartheta \notin \mathbb{Z}$, nous pouvons supposer $y$ assez grand pour que $q \geqslant 2$. Par le Lemme 6.2, nous avons

$$
q\left(n \vartheta ; 2 Q_{y}\right) \geqslant \min \left(q /(n, q), Q_{y} / 2 n\right) .
$$

Soit $a \in \mathbb{Z}^{*}$ tel que $|\vartheta-a / q| \leqslant 1 / q Q_{y}$. Si $q \nmid n$ et $n \leqslant \frac{1}{2} Q_{y}$, alors

$$
|n \vartheta-n a / q| \leqslant n / q Q_{y} \leqslant 1 / 2 q,
$$

donc $\|n \vartheta\| \geqslant 1 / 2 q \geqslant 1 / 2 Q_{y}>1 / y$. Cette minoration et (10.5) nous permettent de déduire du Théorème 2.2 que, si $q \nmid n$,

$$
\nabla_{\mathbf{1}}(n \vartheta ; y) \ll \frac{\sqrt{(n, q) / q}+\sqrt{n / Q_{y}}}{\log y}+\frac{1}{(\log y)^{2}},
$$

et donc, posant $N_{y}:=Q_{y} /(\log y)^{2}$,

$$
\nabla_{\mathbf{1}}(n \vartheta ; y) \ll \frac{\sqrt{(n, q) / q}}{\log y}+\frac{1}{(\log y)^{2}} \quad\left(q \nmid n, 1 \leqslant n \leqslant N_{y}\right) .
$$


En adjoignant à cette estimation la majoration $\nabla_{\mathbf{1}}(n \vartheta ; y) \ll 1$, qui découle trivialement de $(2 \cdot 7)$, et en reportant dans $(10 \cdot 4)$, il suit

$$
\sum_{n \leqslant N_{y}} \frac{\Lambda(n)}{n} \nabla_{\mathbf{1}}(n \vartheta ; y) \ll \frac{S_{1}}{\sqrt{q} \log y}+\frac{1}{\log y}+S_{2},
$$

avec

$$
S_{1}:=\sum_{n \leqslant N_{y}} \frac{\Lambda(n) \sqrt{(n, q)}}{n}, \quad S_{2}:=\sum_{\substack{n \leqslant N_{y} \\ n \equiv 0(\bmod q)}} \frac{\Lambda(n)}{n} .
$$

Maintenant, on observe que

$$
S_{1} \ll \sum_{n \leqslant N_{y}} \frac{\Lambda(n)}{n}+\sum_{p \mid q} \frac{\log p}{\sqrt{p}} \ll \log y+\sqrt{\log q}
$$

alors que

$$
S_{2} \ll \frac{\Lambda(q)}{q} \sum_{p^{\nu}=q, j \geqslant 0} \frac{1}{p^{j}} \ll \frac{\Lambda(q)}{q} .
$$

Nous avons donc obtenu

$$
\sum_{n \leqslant N_{y}} \frac{\Lambda(n)}{n} \nabla_{\mathbf{1}}(n \vartheta ; y) \ll \frac{1}{\sqrt{q}}+\frac{1}{\log y} .
$$

Comme on a clairement

$$
\sum_{\substack{p^{\nu}>N_{y} \\ \nu \geqslant 2}} \frac{\log p}{p^{\nu}} \nabla_{\mathbf{1}}\left(p^{\nu} \vartheta\right) \ll \sum_{\substack{p^{\nu}>N_{y} \\ \nu \geqslant 2}} \frac{\log p}{p^{\nu}} \ll \frac{1}{\log y},
$$

il reste seulement à estimer la contribution correspondant à $n=p \in] N_{y}, y$ ] dans (10.4). À cette fin, nous observons qu'il existe au plus un nombre premier $\left.\left.p_{0} \in\right] N_{y}, y\right]$ tel que $\left\|p_{0} \vartheta\right\|<1 / 2 y$. En effet, pour un tel premier $p_{0}$, il existe $a_{0} \in \mathbb{Z}$ tel que $p_{0} \nmid a_{0}$ et $\left|p_{0} \vartheta-a_{0}\right| \leqslant 1 / 2 y$. Alors, pour tout premier $p \neq p_{0} \leqslant y$, on a

$$
\left|p \vartheta-\frac{a_{0} p}{p_{0}}\right| \leqslant \frac{p}{2 p_{0} y} \leqslant \frac{1}{2 p_{0}},
$$

d'où $\|\vartheta p\| \geqslant 1 / 2 p_{0} \geqslant 1 / 2 y$. Le Théorème 2.2 implique donc

$$
\nabla_{\mathbf{1}}(p \vartheta ; y) \ll 1 / \log y
$$

pour $p \neq p_{0}, N_{y}<p \leqslant y$. Il suit

$$
\sum_{N_{y}<p \leqslant y} \frac{\log p}{p} \nabla_{\mathbf{1}}(p \vartheta ; y) \ll \frac{\log _{2} y}{\log y} .
$$


En sommant $(10 \cdot 8),(10 \cdot 9)$ et $(10 \cdot 10)$, nous obtenons

$$
\sum_{P(n) \leqslant y} \frac{\Lambda(n)}{n} \nabla_{\mathbf{1}}(n \vartheta ; y) \ll \frac{1}{\sqrt{q}}+\frac{\log _{2} y}{\log y} .
$$

Comme $q=q\left(\vartheta ; Q_{y}\right)$ tend vers l'infini avec $y$, cela implique bien (10·4).

Fin de la démonstration du Théorème 4.3.

Nous employons le critère (a) de la Proposition 1.4. Il nous reste donc à établir que la série $U(\log ; \vartheta)$ est $P$-régulière et que $\lim _{y \rightarrow \infty} V_{\Lambda}(\vartheta ; y)=0$.

Le théorème des nombres premiers implique immédiatement

$$
V_{\Lambda}(\vartheta ; y) \ll \sum_{\substack{p \leqslant y \\ p^{\nu}>y}} \frac{\log p}{p^{\nu}} \ll \frac{1}{\sqrt{y}} .
$$

La série $U(\log ; \vartheta)$ est convergente en vertu du critère d'Abel. Sa $P$-régularité équivaut donc à

$$
U_{\log }(\vartheta ; y)=o(1) \quad(y \rightarrow \infty) .
$$

Nous allons établir que, lorsque $q:=q\left(\vartheta ; y /(\log y)^{4 A+28}\right) \geqslant 2$,

$$
U_{\log }(\vartheta ; y) \ll \frac{2^{\omega(q)}(\log q)^{2}}{\varphi(q)}+\frac{1}{(\log y)^{A}}
$$

Cela implique bien que $U_{\log }(\vartheta ; y)=o(1)$ lorsque $y \rightarrow \infty$ pour chaque irrationnel $\vartheta$ fixé, et fournit donc la conclusion attendue.

Une sommation d'Abel permet d'écrire

$$
U_{\log }(\vartheta ; y)=\int_{y}^{\infty} Z(x, y ; \vartheta) \frac{\log x-1}{x^{2}} \mathrm{~d} x+\frac{\log y}{y} Z(y, y ; \vartheta) .
$$

La majoration triviale $Z(y, y ; \vartheta) \ll 1 /\|\vartheta\|$ implique, sous la condition indiquée, que le second terme du membre de droite est $\ll 1 /(\log y)^{B-1}$. Nous évaluons l'intégrale en utilisant (8.12) dans le domaine $y \leqslant x \leqslant Y_{1}$ et en faisant appel au Lemme 6.1 pour le domaine complémentaire $x>Y_{1}$. Nous obtenons bien (10·12). 


\section{Le cas $(f, g)=(\tau, 1):$ preuve du Théorème 4.4}

\subsection{Réduction préliminaire}

Le cas $\vartheta \in \mathbb{Q}$ résulte immédiatement du Théorème 4.7 avec $g=\mathbf{1}$. Les détails sont fournis au paragraphe 13.2. Dans toute la suite de cette démonstration, nous supposons donc $\vartheta \in \mathbb{R} \backslash \mathbb{Q}$.

Nous montrons d'abord que la preuve du Théorème 4.4 peut être réduite au résultat suivant.

Proposition 11.1. Soient $\vartheta \in \mathbb{R} \backslash \mathbb{Q}$ et $\left\{q_{m}\right\}_{m \geqslant 1}$ la suite des dénominateurs des réduites de $\vartheta$. Les trois conditions suivantes sont équivalentes

(i) La série $U(\tau ; \vartheta)$ converge;

(ii) La série $V(\mathbf{1} ; \vartheta)$ converge ;

(iii) La série $\sum_{m \geqslant 1}(-1)^{m}\left(\log q_{m+1}\right) / q_{m}$ converge.

Déduction du Théorème 4.4 à partir de la Proposition 11.1.

Le critère du Théorème 4.4 coïncide avec la condition (iii) de la Proposition 11.1. Cette condition est donc bien nécessaire à la validité de $\left(D_{\vartheta}\right)$ pour le couple $(\tau, \mathbf{1})$. Pour montrer qu'elle est suffisante, nous utilisons le critère (c) de la Proposition 1.4. La convergence de (4.12) implique $\log q_{m+1}=o\left(q_{m}\right)(m \rightarrow \infty)$, donc le Théorème 3.5(a) appliqué à la fonction $\mathbf{1}$ fournit la $\vartheta$-adaptation. Le Lemme 13.2 infra, qui relève d'une technique spécifique aux fonctions multiplicatives, implique que $V_{\mathbf{1}}(\vartheta ; y)=o(1)$ dès que $q\left(\vartheta ; y /(\log y)^{14}\right) \geqslant(\log y)^{14}$. Or, le Lemme 6.9 fournit $U_{\tau}(\vartheta ; y)=o(1)$ lorsque $q\left(\vartheta ; y /(\log y)^{37}\right) \geqslant(\log y)^{37}$. La condition (c) de la Proposition 1.4 est donc satisfaite.

\subsection{Convergence de $U(\tau ; \vartheta)$}

Lemme 11.2. Soit $A>0$. On a uniformément pour $x \geqslant 2, Q_{x}:=x /(\log x)^{4 A+24}$, $\vartheta \in \mathbb{R}, q=q\left(\vartheta ; Q_{x}\right), a \in \mathbb{Z},(a, q)=1,|q \vartheta-a| \leqslant 1 / Q_{x}, \vartheta_{q}:=\vartheta-a / q$,

$$
\begin{aligned}
& Z_{\tau}(x, x ; \vartheta) \\
& \quad=x(\log x)\left\{\frac{\sin ^{2}\left(\pi \vartheta_{q} x\right)}{\pi q \vartheta_{q} x}+O\left(\frac{(\log q) \log \left(1+\left(\vartheta_{q} x\right)^{2}\right)}{q\left|\vartheta_{q}\right| x \log x}+\frac{1}{(\log x)^{A}}\right)\right\} .
\end{aligned}
$$

Démonstration. Les Lemmes 6.8 et 6.10 appliqués avec $z=2$ fournissent

$$
E_{\tau}(x, x ; a / q)=\frac{x}{q}(\log x+2 \gamma-1-2 \log q)+O\left(\frac{x}{(\log x)^{A}}\right)
$$

uniformément pour $x \geqslant 2,1 \leqslant q \leqslant x /(\log x)^{4 A+24},(a, q)=1$. $^{(20)}$ La formule asymptotique annoncée est déduite de $(11 \cdot 2)$ par sommation d'Abel, comme dans

\footnotetext{
20. Jutila a montré dans [16] que, pour chaque $\varepsilon>0$ fixé, on a la formule uniforme

$E_{\tau}(x, x ; a / q)=\frac{x}{q}(\log x+2 \gamma-1-2 \log q)+O\left(q^{2 / 3} x^{1 / 3+\varepsilon}\right) \quad(x \geqslant 2, q \geqslant 1,(a, q)=1)$.

Nous n'avons pas besoin d'une telle précision ici.
} 
la preuve du Lemme 6.11. En fait (11.1) coïncide avec (6.41) dans le cas $z=2$ à l'exception du facteur $\log q$ dans le premier terme résiduel, qui est remplacé par $q^{\varepsilon}$ dans (6-41). Nous omettons la vérification de cette légère amélioration.

Preuve de l'équivalence des points (i) et (iii) de la Proposition 11.1.

Nous sommes maintenant en mesure d'établir l'équivalence des points (i) et (iii) de la Proposition 11.1. Nous utilisons les notations de (6·3). D'après le Lemme 11.2, on a

$$
\begin{aligned}
\int_{\xi_{m}}^{\xi_{m+1}} Z_{\tau}(t, t ; \vartheta) \frac{\mathrm{d} t}{t^{2}}= & \frac{1}{q_{m}} \int_{\xi_{m}}^{\xi_{m+1}} \frac{\left(\sin \pi \varepsilon_{m} t\right)^{2} \log t}{\pi \varepsilon_{m} t^{2}} \mathrm{~d} t \\
& +O\left(\frac{\log q_{m}}{q_{m}}+\int_{\xi_{m}}^{\xi_{m+1}} \frac{\mathrm{d} t}{t(\log t)^{A}}\right) \\
= & -\operatorname{sgn}\left(\varepsilon_{m}\right) \frac{\log \left|\varepsilon_{m}\right|}{q_{m}} \int_{\left|\varepsilon_{m}\right| \xi_{m}}^{\left|\varepsilon_{m}\right| \xi_{m+1}} \frac{(\sin \pi u)^{2}}{\pi u^{2}} \mathrm{~d} u \\
& +O\left(\frac{\log q_{m}}{q_{m}}+\int_{\xi_{m}}^{\xi_{m+1}} \frac{\mathrm{d} t}{t(\log t)^{A}}\right),
\end{aligned}
$$

où, pour établir la seconde égalité, nous avons utilisé l'intégrabilité sur $\mathbb{R}$ de la fonction $(\sin \pi u)^{2}(\log u) / u^{2}$. De plus, le même calcul fournit

$$
\int_{\xi_{m}}^{x} Z_{\tau}(t, t ; \vartheta) \frac{\mathrm{d} t}{t^{2}} \ll \frac{\log q_{m+1}}{q_{m}}+\int_{\xi_{m}}^{\xi_{m+1}} \frac{\mathrm{d} t}{t(\log t)^{A}} \quad\left(\xi_{m}<x \leqslant \xi_{m+1}\right) .
$$

Grâce à (6·3), nous avons encore

$$
\int_{\left|\varepsilon_{m}\right| \xi_{m+1}}^{+\infty} \frac{(\sin \pi u)^{2}}{\pi u^{2}} \mathrm{~d} u \ll \frac{1}{1+\left|\varepsilon_{m}\right| \xi_{m+1}} \ll \frac{q_{m}}{q_{m}+\left(\log q_{m+1}\right)^{B}} \ll \frac{q_{m}^{1 / B}}{\log q_{m+1}}
$$

et

$$
\int_{0}^{\left|\varepsilon_{m}\right| \xi_{m}} \frac{(\sin \pi u)^{2}}{\pi u^{2}} \mathrm{~d} u \ll\left|\varepsilon_{m}\right| \xi_{m} \ll \frac{\left(\log q_{m}\right)^{B}}{q_{m+1}} .
$$

Compte tenu de la formule classique

$$
\int_{0}^{\infty} \frac{(\sin \pi u)^{2}}{\pi u^{2}} \mathrm{~d} u=\frac{1}{2} \pi
$$

nous déduisons donc de (11.3) que

$$
\begin{aligned}
\int_{\xi_{m}}^{\xi_{m+1}} Z_{\tau}(t, t ; \vartheta) \frac{\mathrm{d} t}{t^{2}}= & \frac{1}{2} \pi \operatorname{sgn}\left(\varepsilon_{m}\right) \frac{\log q_{m+1}}{q_{m}} \\
& +O\left(\frac{1}{q_{m}^{1-1 / B}}+\int_{\xi_{m}}^{\xi_{m+1}} \frac{\mathrm{d} t}{t(\log t)^{A}}\right) .
\end{aligned}
$$


Choisissons $A>1$, de sorte que l'intégrale $\int_{2}^{\infty} \mathrm{d} t / t(\log t)^{A}$ converge. Comme

$$
\sum_{m \geqslant 1} q_{m}^{-1+1 / B}<\infty
$$

en vertu de la croissance exponentielle des $q_{m}$, on voit que le terme d'erreur de (11.5) est le terme général d'une série convergente. Comme $\operatorname{sgn} \varepsilon_{m}=(-1)^{m} \operatorname{sgn} \varepsilon_{1}$, nous obtenons que la convergence de la série $\sum_{m \geqslant 1} \int_{\xi_{m}}^{\xi_{m+1}} Z_{\tau}(t, t ; \vartheta) \mathrm{d} t / t^{2}$ équivaut à celle de la série apparaissant au point (iii) de l'énoncé.

Considérons maintenant un nombre réel $x \geqslant 2$ et désignons par $M=M_{x}$ l'unique entier tel que $\xi_{M} \leqslant x<\xi_{M+1}$. On a par (11.4)

$$
\begin{aligned}
\sum_{n \leqslant x} \frac{\tau(n)}{n} & \sin (2 \pi n \vartheta)=\frac{Z_{\tau}(x, x ; \vartheta)}{x}+\int_{1}^{x} Z_{\tau}(t, t ; \vartheta) \frac{\mathrm{d} t}{t^{2}} \\
& =\sum_{1 \leqslant m \leqslant M} \int_{\xi_{m}}^{\xi_{m+1}} Z_{\tau}(t, t ; \vartheta) \frac{\mathrm{d} t}{t^{2}}+O\left(\frac{\log q_{M+1}}{q_{M}}\right)+o(1)
\end{aligned}
$$

Sous la condition (iii), on a $\lim _{m \rightarrow \infty}\left(\log q_{m+1}\right) / q_{m}=0$. On déduit donc de ce qui précède l'implication (iii) $\Rightarrow$ (i).

Établissons la réciproque. Au vu de (11.6) et (11.5), il suffit de prouver que la convergence de $U(\tau ; \vartheta)$ implique

$$
\log q_{m+1}=o\left(q_{m}\right) \quad m \rightarrow \infty
$$

Or on a

$$
\sum_{\xi_{m}<n \leqslant \xi_{m+1}} \frac{\tau(n)}{n} \sin (2 \pi n \vartheta)=\left[\frac{Z_{\tau}(x, x ; \vartheta)}{x}\right]_{\xi_{m}}^{\xi_{m+1}}+\int_{\xi_{m}}^{\xi_{m+1}} Z_{\tau}(t, t ; \vartheta) \frac{\mathrm{d} t}{t^{2}}
$$

La formule $(11 \cdot 1)$ et les relations $(6 \cdot 3)$ permettent de montrer facilement que le premier terme du membre de droite est $\ll\left(\log q_{m}\right) / q_{m}=o(1)(m \rightarrow \infty)$. Sous l'hypothèse (i), le membre de gauche tend vers 0 puisque c'est le terme général d'une série convergente. On déduit donc (11·7) de (11.5).

\subsection{Convergence de $V(1 ; \vartheta)$}

Nous commençons par estimer $W_{\mathbf{1}}(x, x ; \vartheta)$, en vue d'intégrations par parties ultérieures.

Lemme 11.3. Soit $A>0$. On a uniformément pour $x \geqslant 2, Q_{x}=x /(\log x)^{5 A+20}$, $\vartheta \in \mathbb{R}, q:=q\left(\vartheta ; Q_{x}\right), a \in \mathbb{Z},(a, q)=1,|q \vartheta-a| \leqslant 1 / Q_{x}, \vartheta_{q}:=\vartheta-a / q$,

$$
W_{\mathbf{1}}(x, x ; \vartheta)=\frac{-x}{2 q}\left\{\operatorname{sgn}\left(\vartheta_{q}\right)+O(x\|q \vartheta\|)\right\}+O\left(\frac{x}{(\log x)^{A}}\right) \quad(x\|q \vartheta\| \leqslant 1)
$$


et

$$
W_{\mathbf{1}}(x, x ; \vartheta) \ll \frac{1}{q\|q \vartheta\|}+\frac{x}{(\log x)^{A}} \quad(x\|q \vartheta\|>1) .
$$

Démonstration. Le cas $\vartheta_{q}=0$ est trivial. En effet, seule la relation (11.8) est alors à démontrer, et il résulte du fait que $n \mapsto B(a n / q)$ est de moyenne nulle dans $\mathbb{Z} / q \mathbb{Z}$ que

$$
W_{\mathbf{1}}(x, x ; a / q) \ll x-q[x / q] \ll q .
$$

Plaçons-nous donc dans le cas $\vartheta_{q} \neq 0$. Soit $B:=5 A+20$, de sorte que $Q_{x}=x /(\log x)^{B}$. Si $q=q\left(\vartheta ; Q_{x}\right)>(\log x)^{B}$, la majoration $(7 \cdot 4)$ fournit à la fois $(11 \cdot 8)$ et $(11 \cdot 9)$. Nous supposons donc dans la suite que $q \leqslant(\log x)^{B}$. Comme les deux membres de $(11.8)$ sont des fonctions impaires de $\vartheta$, nous pouvons également, sans restreindre la généralité, supposer que $\vartheta_{q}>0$.

Appliquons le Lemme 7.5 à la fonction 1. Nous pouvons écrire, avec les notations $(7 \cdot 16)$ pour $f=\mathbf{1}$,

$$
W_{\mathbf{1}}(x, x ; \vartheta)=\sum_{0 \leqslant b \leqslant x\|q \vartheta\|} T_{b}+O\left((\log x)^{B}\right)
$$

avec $T_{b}:=T_{b}^{(1)}+T_{b}^{(2)}-\frac{1}{2} T_{b}^{(3)}$, où

$$
\begin{aligned}
T_{b}^{(1)} & =\sum_{x_{b}<n \leqslant x_{b+1}} B\left(\frac{a n+b}{q}\right)=\sum_{1 \leqslant c \leqslant q} B\left(\frac{a c+b}{q}\right)\left(\frac{x_{b+1}-x_{b}}{q}+O(1)\right) \ll q, \\
T_{b}^{(2)} & =\sum_{x_{b}<n \leqslant x_{b+1}}\left(n \vartheta_{q}-\frac{b}{q}\right)=\frac{1}{2} \vartheta_{q}\left(x_{b+1}-x_{b}\right)^{2}+O\left((\log x)^{B}\right), \\
T_{b}^{(3)} & =\sum_{\substack{x_{b}<n \leqslant x_{b+1} \\
a n \equiv-b(\bmod q)}} 1=\frac{x_{b+1}-x_{b}}{q}+O(1) .
\end{aligned}
$$

Il reste à sommer ces évaluations. Si $x \leqslant 1 /\|q \vartheta\|$, on obtient

$$
W_{\mathbf{1}}(x, x ; \vartheta)=T_{0}=-\frac{x}{2 q}+O\left(\frac{\|q \vartheta\| x^{2}}{q}+(\log x)^{B}\right),
$$

ce qui implique bien $(11 \cdot 8)$. Si $x>1 /\|q \vartheta\|$, on a $\|q \vartheta\|\left(x_{b+1}-x_{b}\right)=1$ pour tout $b$ tel que $b+1 \leqslant x\|q \vartheta\|$, d'où, sous cette condition,

$$
T_{b}=O(q)+\frac{\vartheta_{q}}{2\|q \vartheta\|^{2}}-\frac{1}{2 q\|q \vartheta\|}+O\left((\log x)^{B}\right) \ll(\log x)^{B} .
$$

Comme on déduit aussi de nos estimations des $T_{b}^{(j)}$ que

il s'ensuit finalement que

$$
\max _{b}\left|T_{b}\right| \ll \frac{1}{q\|q \vartheta\|}+(\log x)^{B}
$$

$$
W_{\mathbf{1}}(x, x ; \vartheta) \ll \frac{1}{q\|q \vartheta\|}+(\log x)^{2 B} \quad(x>1 /\|q \vartheta\|),
$$

ce qui implique bien (11.9). 
Preuve de l'équivalence des points (ii) et (iii) de la Proposition 11.1.

Une sommation d'Abel permet d'écrire

$$
\sum_{1 \leqslant n \leqslant x} \frac{B(n \vartheta)}{n}=\frac{W_{\mathbf{1}}(x, x ; \vartheta)}{x}+\int_{1}^{x} W_{\mathbf{1}}(t, t ; \vartheta) \frac{\mathrm{d} t}{t^{2}} .
$$

En notant que $\left\|q_{m} \vartheta\right\|=\left|\varepsilon_{m}\right| q_{m}$, on déduit du Lemme 11.3 que

$$
\begin{aligned}
\int_{\xi_{m}}^{\xi_{m+1}} W_{\mathbf{1}}(t, t ; \vartheta) & \frac{\mathrm{d} t}{t^{2}}=-\frac{\operatorname{sgn}\left(\varepsilon_{m}\right)}{2 q_{m}} \int_{\xi_{m}}^{1 /\left\|q_{m} \vartheta\right\|} \frac{\mathrm{d} t}{t}+O\left(\frac{1}{q_{m}}+\int_{\xi_{m}}^{\xi_{m+1}} \frac{\mathrm{d} t}{t(\log t)^{A}}\right) \\
& =(-1)^{m+1} \operatorname{sgn}\left(\varepsilon_{1}\right) \frac{\log q_{m+1}}{2 q_{m}}+O\left(\frac{\log q_{m}}{q_{m}}+\int_{\xi_{m}}^{\xi_{m+1}} \frac{\mathrm{d} t}{t(\log t)^{A}}\right),
\end{aligned}
$$

où l'on a utilisé les estimations (6·3). De la même manière, on obtient

$$
\frac{W_{\mathbf{1}}(x, x ; \vartheta)}{x} \ll \frac{1}{\left(\log q_{m}\right)^{A}}, \quad \int_{\xi_{m}}^{x} W_{\mathbf{1}}(t, t ; \vartheta) \frac{\mathrm{d} t}{t^{2}} \ll \frac{\log q_{m+1}}{q_{m}} \quad\left(\xi_{m}<x \leqslant \xi_{m+1}\right) .
$$

Ces estimations sont suffisantes pour obtenir l'équivalence requise des conditions (ii) et (iii). Nous omettons les détails finaux, qui sont identiques à ceux du paragraphe 11.2.

\section{2. $\left(D_{\vartheta}\right)$ pour $(f, g)=(\Lambda,-\mu \log )$ : preuve du Théorème 4.5}

Nous employons la Proposition 1.4(b). La $\vartheta$-adaptation, soit

$$
\nabla_{\Lambda}(\vartheta ; y)=\sum_{P(m) \leqslant y} \frac{\Lambda(m)}{m} \nabla(m \vartheta ; y)=o(1) \quad(y \rightarrow \infty)
$$

est déduite de $(2 \cdot 3)$ de la même manière que celle du couple $(\log , \Lambda)$ l'est de $(2 \cdot 7)$. Nous renvoyons le lecteur au Lemme 10.1 pour les détails. La $P$-régularité de la série

$$
V(\mu \log ; \vartheta)=\sum_{n \geqslant 1} \frac{\mu(n) \log n}{n} B(n \vartheta)
$$

est une conséquence banale, par sommation d'Abel, des estimations (7·3) ou (7·38) de $W_{\mu}(x, y ; \vartheta)$ - nous omettons les calculs correspondants. Comme on a

$$
\left|U_{\Lambda}(\vartheta ; y)\right| \leqslant \sum_{\substack{P(m) \leqslant y \\ m>y}} \frac{\Lambda(m)}{m} \ll \frac{1}{\sqrt{y}} \quad(y \geqslant 2)
$$

d'après une majoration classique, toutes les conditions de la Proposition 1.4(b) sont bien remplies. 
Il reste à montrer que la convergence des deux membres de $\left(D_{\vartheta}\right)$ est uniformément bornée pour $(f, g)=(\Lambda,-\mu \log )$. C'est une conséquence immédiate de $(1 \cdot 7)$, par sommation d'Abel, dans le cas de $V(g ; \vartheta)$. Il suffit donc, au vu de $(1 \cdot 16)$ de montrer que les trois quantités $\nabla_{\Lambda}(\vartheta ; y), U_{\Lambda}(\vartheta ; y)$ et $V_{g}(\vartheta ; y)$ sont uniformément bornées en $y$ et $\vartheta$. La première propriété résulte de $(12 \cdot 1)$ et de la majoration $\sup _{\vartheta}|\nabla(\vartheta ; y)| \ll 1 / \log y$ du Théorème 2.1. La seconde découle de (12.2) et la troisième de $(7 \cdot 3)$, par sommation d'Abel.

\section{Cas des fonctions multiplicatives}

\subsection{Preuve du Théorème 4.6 $:(f, g) \in \mathcal{F}_{A} \times \mathcal{F}_{A}$ et $\vartheta \in \mathbb{R} \backslash \mathbb{Q}$}

Nous établissons le Théorème 4.6 en faisant appel au critère (a) de la Proposition 1.4. Il est donc nécessaire de disposer d'une majoration de $V_{g}(\vartheta ; y)$. Nous ferons appel, à cette fin, à un théorème de Montgomery \& Vaughan [22] qui implique que, pour chaque $A \geqslant 1$,

$$
E_{g}(x, y, \vartheta) \ll_{A} \frac{x}{\log x}+x \frac{(\log R)^{3 / 2}}{R^{1 / 2}} \quad(x \geqslant y \geqslant 2),
$$

uniformément pour $g \in \mathcal{F}_{A}, \vartheta \in \mathbb{R}, R \geqslant 2, q(\vartheta ; x / R) \geqslant R$. Il est à noter que nous avons utilisé ici un aspect essentiel du théorème de Montgomery \& Vaughan, à savoir l'uniformité de l'estimation dans la classe $\mathcal{F}_{A}$ : cela permet, en appliquant le résultat à la fonction multiplicative $g \mathbf{1}_{S(\infty, y)}$, d'estimer indépendamment de $y$ des sommes portant sur $S(x, y)$.

Bien entendu, en raison même de son caractère d'uniformité, la majoration $(13 \cdot 1)$ n'est pertinente que pour des «grandes» valeurs de $y$. Lorsque $y$ est petit devant $x$, nous remplaçons $(13 \cdot 1)$ par l'estimation

$$
E_{g}(x, y ; \vartheta) \ll A^{2} x(\log x)^{3 / 2}\left\{\sqrt{\frac{y}{x}}+\frac{1}{\sqrt{R}}+\mathrm{e}^{-\sqrt{\log x}}\right\}
$$

établie dans [2] (proposition 1), avec les mêmes conditions de validité que $(13 \cdot 1)$ en $x, y, g, R$ et $\vartheta$. Il est en outre à noter que la constante implicite dans $(13 \cdot 2)$ est absolue, et donc que le paramètre $A$ est en particulier autorisé à dépendre de $x$.

En cohérence avec la notation $(7 \cdot 1)$, nous posons, pour $x \geqslant 2, y \geqslant 2, \vartheta \in \mathbb{R}$,

$$
W_{g}(x, y ; \vartheta):=\sum_{n \in S(x, y)} g(n) B(n \vartheta) .
$$

Lemme 13.1. Soit $A \geqslant 1$. On a uniformément pour $g \in \mathcal{F}_{A}, x \geqslant y \geqslant 2, \vartheta \in \mathbb{R}$, $R \geqslant 1, q(\vartheta ; x / R) \geqslant R$,

$$
W_{g}(x, y ; \vartheta) \ll x \frac{\log _{2} x}{\log x}+x \frac{\log R}{R^{1 / 3}}
$$


et

$$
W_{g}(x, y ; \vartheta) \ll x(\log x)^{5 / 2}\left\{\sqrt{\frac{y}{x}}+\frac{1}{R^{1 / 3}}+\mathrm{e}^{-\sqrt{\log x}}\right\} .
$$

Démonstration. Nous réemployons ici la technique utilisée au Lemme 7.2 pour $g=\tau_{z}$, et qui consiste à approcher $B(\vartheta)$ par une série de Fourier absolument convergente. Le rôle des estimations (6.32) est joué par (13.1) pour la preuve de (13.4) et par (13.2) pour celle de (13.5). Comme les détails techniques sont essentiellement identiques à ceux de la preuve du Lemme 7.2, nous nous contentons de brèves indications.

Avec les notations $(7 \cdot 6)$ et en posant $K_{h}(x, y ; \vartheta):=\sum_{n \in S(x, y)} h(n) \cos (2 \pi n \vartheta)$, nous avons pour $M \geqslant 1,0<\delta<1$,

$$
\begin{aligned}
W_{g}(x, y ; \vartheta)= & \sum_{1 \leqslant m \leqslant M} b_{\delta, m} Z_{g}(x, y ; m \vartheta) \\
& +O\left(\sum_{1 \leqslant m \leqslant M} a_{\delta, m} K_{|g|}(x, y ; m \vartheta)+\Psi(x, y)\{\delta+1 / M \delta\}\right) .
\end{aligned}
$$

En employant la minoration $q(m \vartheta ; 2 x / R) \geqslant R / m$ issue du Lemme 6.2, nous déduisons de (13.6) et (13.1) que l'on a, sous les hypothèses indiquées et pour $2 \leqslant M \leqslant \frac{1}{2} R$,

$$
W_{g}(x, y ; \vartheta) \ll \frac{x \log M}{\log x}+\delta x+\frac{x}{\delta M}+\frac{x(\log R)^{3 / 2}}{\sqrt{\delta R}} .
$$

Nous en déduisons (13·4) en choisissant

$$
\delta:=\frac{\log R}{\sqrt[3]{R}}+\frac{1}{(\log x)^{3}}, \quad M:=\min \left\{\frac{1}{2} R,(\log x) / \delta\right\} .
$$

Nous procédons parallèlement pour établir (13.5), en utilisant (13.2) au lieu de $(13 \cdot 1)$. Le résultat énoncé correspond au choix $M:=\frac{1}{2} R, \delta:=1 / \sqrt[3]{R}$.

Nous déduisons à présent du Lemme 13.1 un résultat que l'on peut qualifier de $B$-analogue du théorème 2 de [3].

Lemme 13.2. Soit $A \geqslant 1$. On a uniformément sous les conditions $g \in \mathcal{F}_{A}, y \geqslant 2$, $Q_{y}:=y /(\log y)^{14}, \vartheta \in \mathbb{R}, q\left(\vartheta ; Q_{y}\right) \geqslant(\log y)^{14}$,

$$
V_{g}(\vartheta ; y) \ll \frac{\left(\log _{2} y\right)^{2}}{\log y} .
$$

Démonstration. Évaluons, sous l'hypothèse $q(\vartheta ; y / R) \geqslant R$, le membre de gauche de (13.7) par sommation d'Abel en utilisant (13.4) pour $y \leqslant x \leqslant R y,(13 \cdot 5)$ pour 
$R y<x \leqslant Y_{1}$ et la majoration triviale $W_{g}(x, y ; \vartheta) \ll \Psi(x, y)$ pour $x>Y_{1}$. Nous obtenons, compte tenu du Lemme 6.1,

$$
V_{g}(\vartheta ; y)=\sum_{\substack{P(n) \leqslant y \\ n>y}} \frac{g(n)}{n} B(\vartheta n) \ll \frac{\left(\log _{2} y\right)^{2}}{\log y}+\frac{(\log y)^{7 / 2}\left(\log _{2} y\right)^{7 / 2}}{R^{1 / 3}}
$$

Pour $R=(\log y)^{14}$, le premier terme de cette majoration domine.

Nous reformulons qualitativement le Lemme 13.2 sous forme d'un $B$-analogue du corollaire 2.1 de [3].

Corollaire 13.3. Soient $A>0, g \in \mathcal{F}_{A}$ et $\vartheta \in \mathbb{R} \backslash \mathbb{Q}$. Si la série $V(g ; \vartheta)$ est convergente et $P$-convergente, alors elle est $P$-régulière.

Démonstration. Soient $\alpha$ la somme et $\alpha^{*}$ la $P$-somme de la série $V(g ; \vartheta)$. On a clairement $\alpha-\alpha^{*}=V_{g}(\vartheta ; y)+o(1)$, donc $\alpha=\alpha^{*}$ dès que $\liminf _{y \rightarrow \infty}\left|V_{g}(\vartheta ; y)\right|=0$. Cette dernière condition résulte immédiatement du Lemme 13.2 puisque, si $\vartheta$ est irrationnel, on a $q(\vartheta ; y)=y$ pour une infinité d'entiers $y$.

Fin de la démonstration du Théorème 4.6.

Nous utilisons le critère (c) de la Proposition 1.4. Comme $\mathcal{F}_{A} \subset \mathcal{L}^{2}$, le Théorème 3.1 implique que le couple $(f, g)$ est $\vartheta$-adapté. D'après le Lemme 13.2 , nous avons $V_{g}(\vartheta ; y)=o(1)$ dès que $q\left(\vartheta ; y /(\log y)^{14}\right) \geqslant(\log y)^{14}$. Or, le théorème 2 de [3] implique $U_{f}(\vartheta ; y)=o(1)$ sous la même condition. La condition (c) de la Proposition 1.4 est donc bien remplie.

\subsection{Preuve du Théorème 4.7:g $\in \mathcal{F}_{A}^{*}$ et $\vartheta \in \mathbb{Q}$}

Nous utilisons la Proposition 1.4(b).

La $\vartheta$-adaptation résulte immédiatement du Théorème 3.4 puisque les hypothèses effectuées sur $g$ impliquent facilement

$$
\sum_{P(n) \leqslant y} \frac{|g(n)|}{n} \ll \log y, \quad \sum_{P(m) \leqslant y} \frac{|f(m)|}{m} \ll(\log y)^{2},
$$

où la première des ces deux majorations a été établie en raisonnant comme dans la preuve de $(9 \cdot 10)$ avec $\alpha=\beta=2$.

Pour établir la $P$-régularité de la série $V(g ; \vartheta)$, nous observons d'abord que l'espace vectoriel des fonctions de $\mathbb{Z}$ dans $\mathbb{C}$ qui sont $q$-périodiques et impaires admet les familles génératrices suivantes :

l'ensemble des caractères $\chi$ impairs dont le module divise $q$;

l'ensemble des fonctions $n \mapsto \sin (2 \pi b n / q), b \in \mathbb{N}$;

l'ensemble des fonctions $n \mapsto B(b n / q), b \in \mathbb{N}$.

Cela implique que $V(g ; \vartheta)$ est combinaison linéaire de séries du type

$$
\sum_{n \geqslant 1} g(n) \chi(n) / n
$$


où $\chi$ est un caractère de Dirichlet impair de module divisant $q$. Un théorème de Montgomery (voir [14], théorème 12) stipule que ces séries sont $P$-régulières dès qu'elles sont convergentes si $g \in \mathcal{M}_{1}$ et si les séries (4.16) sont toutes non nulles lorsque $p=2$ - ce qui est alors équivalent à l'existence d'un entier $\nu \geqslant 1$ tel que $g\left(2^{\nu}\right) \chi\left(2^{\nu}\right) \neq 0$. Or, la démonstration donnée dans [14] du résultat de Montgomery s'étend sans difficulté au cas des séries (13.8) avec $g \in \mathcal{F}_{A}^{*}$ sous l'hypothèse $(4 \cdot 16)$. L'hypothèse (a) implique donc la $P$-régularité de $V(g ; \vartheta)$.

Il reste à montrer que

$$
U_{f}(\vartheta ; y)=o(1) \quad(y \rightarrow \infty)
$$

À cette fin, nous faisons appel à (4·6), sous la forme

$$
U_{f}(\vartheta ; y)=\sum_{n \leqslant y} \frac{g(n)}{n}\left\{B^{*}\left(n \vartheta ; \frac{y}{n}\right)-B(n \vartheta)+\nabla_{\mathbf{1}}(n \vartheta ; y)\right\} .
$$

La relation $(2 \cdot 4)$ implique

$$
\sup _{n \leqslant y}|\nabla(n \vartheta ; y)| \ll 1 /(\log y)^{A+2}
$$

et la formule $(1 \cdot 22)$ appliquée avec $f=\mathbf{1}$ permet d'en déduire immédiatement une majoration identique pour $\sup _{n \leqslant y}\left|\nabla_{\mathbf{1}}(n \vartheta ; y)\right|$. Cela montre que la contribution des termes $\nabla_{\mathbf{1}}(n \vartheta ; y)$ dans $(13 \cdot 10)$ est négligeable. Introduisons alors un paramètre $T>1$ et écrivons la contribution complémentaire sous la forme

$$
G_{1}(T ; y)+G_{2}(T ; y)
$$

où $G_{1}$ et $G_{2}$ correspondent aux intervalles de sommation respectifs $n \leqslant y / T$ et $y / T<n \leqslant y$. Pour estimer $G_{1}(T ; y)$, nous faisons appel à $(4 \cdot 2)$ sous la forme

$$
B^{*}(n \vartheta ; y / n)-B(n \vartheta) \ll n /(n+y / q) \ll_{\vartheta} n / y .
$$

Insérons cette estimation dans l'expression de $G_{1}(T ; y)$. En utilisant le fait que $g$ est bornée en moyenne quadratique et en appliquant l'inégalité de Cauchy-Schwarz, nous en déduisons que

$$
G_{1}(T ; y) \ll \vartheta 1 / \sqrt{T} .
$$

Nous avons enfin

$$
G_{2}(T ; y)=-\sum_{y / T<n \leqslant y} g(n) \frac{B(a n / q)}{n}-\sum_{1 \leqslant m \leqslant T} \frac{1}{\pi m} \sum_{y / T<n \leqslant y / m} g(n) \frac{\sin (2 \pi a m n / q)}{n} .
$$

Les hypothèses (b) et (c) impliquent donc que $G_{2}(T ; y)=o(1)$ pour chaque $T$ fixé lorsque $y \rightarrow \infty$. Nous obtenons alors (13.9) en faisant tendre successivement $y$ et $T$ vers l'infini. 


\subsection{Preuve du Théorème 4.8 $:(f, g)=\left(\tau_{z+1}, \tau_{z}\right)$}

Nous pouvons aisément disposer du cas $\vartheta \in \mathbb{Q}$, pour lequel le Théorème 3.4 fournit immédiatement la $\vartheta$-adaptation. En effet, en utilisant, comme dans la preuve du Théorème 4.7 , le fait que l'ensemble $\mathcal{D}(q)$ des caractères de Dirichlet impairs de module divisant $q$ constitue une base de l'espace vectoriel des fonctions $q$-périodiques impaires de $\mathbb{Z}$ dans $\mathbb{C}$, nous voyons que la conclusion requise résulte de la $P$-régularité de la série $\sum_{n \geqslant 1} \tau_{w}(n) \chi(n) / n$ pour $\chi \in \mathcal{D}(q)$ et $w \in\{z, z+1\}$. En fait, cette série est $P$-régulière, de somme $L(1, \chi)^{w}$, pour tout $\chi$ non principal et tout $w \in \mathbb{C}$. La $P$-convergence vers $L(1, \chi)^{w}$ est une conséquence bien connue du théorème des nombres premiers en progressions arithmétiques. La convergence au sens ordinaire découle de $(6 \cdot 37)$, grâce à la représentation classique

$$
\chi(n)=G_{q}(\bar{\chi})^{-1} \sum_{1 \leqslant a \leqslant q} \overline{\chi(a)} \mathrm{e}(a n / q) \quad(n \geqslant 1) .
$$

où $G_{q}(\bar{\chi})$ désigne la somme de Gauss associée à $\bar{\chi}$. Le fait que la somme ordinaire soit égale à $L(1, \chi)^{w}$ est alors une conséquence de la continuité de $L(s, \chi)$ sur $[1, \infty[$.

Nous nous limitons donc dans la suite à considérer les nombres $\vartheta$ irrationnels. Le cas $z=0$ étant trivial puisqu'il correspond à la convergence de la série (1.2), nous pouvons supposer $\Re e z<0$. Nous utilisons alors le critère (c) de la Proposition 1.4. Le Théorème 3.1 fournit la $\vartheta$-adaptation de $\left(\tau_{z+1}, \tau_{z}\right)$ dès que $|z+1| \leqslant 1$, puisque $\left|\tau_{w}(n)\right| \leqslant 1$ pour $|w| \leqslant 1$ et $n \geqslant 1$. Le Lemme 6.9 implique ensuite que

$$
U_{\tau_{z+1}}(\vartheta ; y)=o(1)
$$

lorsque $y \rightarrow \infty$ sous la condition $q\left(\vartheta ; y /(\log y)^{C}\right) \geqslant(\log y)^{C}$ avec $C \geqslant 4(\kappa+1)^{2}+21$. Les trois sous-paragraphes suivants sont consacrés à établir que

$$
V_{\tau_{z}}(\vartheta ; y)=o(1)
$$

pour les mêmes valeurs de $y$ et un choix convenable de $C$, et que les séries $V\left(\tau_{z} ; \vartheta\right)$, $U\left(\tau_{z+1} ; \vartheta\right)$ sont respectivement convergentes pour $\Re e z<0$ et $\Re e z \leqslant 0$.

\subsubsection{Majoration de $\left|V_{\tau_{z}}(\vartheta ; y)\right|$}

Le Lemme 7.2 fournit immédiatement une estimation du défaut de $P$-régularité $V_{\tau_{z}}(\vartheta ; y)$.

Lemme 13.4. Soit $\kappa \geqslant 1$. On a uniformément pour $y \geqslant 2, z \in \mathbb{C},|z| \leqslant \kappa, \vartheta \in \mathbb{R}$, $R \geqslant 1, q(\vartheta ; y / R) \geqslant R$,

$$
V_{\tau_{z}}(\vartheta ; y)=\sum_{\substack{P(n) \leqslant y \\ n>y}} \frac{\tau_{z}(n)}{n} B(\vartheta n) \ll(\log y)^{\kappa^{2}+5}\left\{R^{-1 / 5}+\mathrm{e}^{-\frac{1}{2} \sqrt{\log y}}\right\} .
$$

Démonstration. Il suffit d'intégrer par parties l'estimation (7.4) en utilisant la majoration triviale issue de (6.22) pour $x>Y_{1}$. Nous omettons les détails. 
On déduit en particulier de (13.13) que l'on a (13.12) lorsque $y \rightarrow \infty$ sous la condition $q\left(\vartheta ; y /(\log y)^{C}\right) \geqslant(\log y)^{C}$ avec $C \geqslant 5 \kappa^{2}+26$.

Compte tenu du domaine de validité de $(13 \cdot 11)$ rappelé plus haut, nous avons donc établi que, pour tout nombre complexe $z$, la condition (1.18) de la Proposition 1.4(c) est bien vérifiée pour le couple $\left(\tau_{z+1}, \tau_{z}\right)$.

13.3.2. Estimations de $W_{\tau_{z}}(x, y ; \vartheta)$ : convergence de $V\left(\tau_{z} ; \vartheta\right)$

Ce paragraphe est consacré à établir la convergence de la série $V\left(\tau_{z} ; \vartheta\right)$ sous l'hypothèse $\Re e z<0$.

Le résultat intermédiaire suivant nous sera utile.

Lemme 13.5. Soient $A>0, B>0, \kappa \geqslant 1$. On a uniformément pour $x \geqslant 2$, $1 \leqslant w \leqslant x, 1 \leqslant r \leqslant(\log x)^{B}, 1 \leqslant m \leqslant(\log x)^{B},(c, m)=1,|z| \leqslant \kappa$,

$$
\begin{aligned}
\sum_{\substack{x<n \leqslant x+x / w \\
n \equiv c(\bmod m)}} \tau_{z}(r n) & =\frac{1}{\varphi(m)} \sum_{\substack{x<n \leqslant x+x / w \\
(n, m)=1}} \tau_{z}(r n)+O\left(\frac{x}{(\log x)^{A}}\right) \\
& \ll \frac{\tau_{\kappa}(r) \log (2 r m)}{m} \frac{x}{w}(\log x)^{\Re e z-1}+\frac{x}{(\log x)^{A}} .
\end{aligned}
$$

En particulier, on a $\tau_{z} \in S W^{+}$pour tout $z \in \mathbb{C}$, les constantes implicites de $(7 \cdot 12)$ et (7.13) pour $f=\tau_{z}$ étant localement uniformes en $z$.

Démonstration. Le résultat annoncé pourrait être déduit formellement du Lemme 6.10, mais il est plus simple de procéder directement. Nous nous contentons d'indiquer les étapes essentielles.

Désignons par $\chi$ un caractère générique de module $m$ et posons

$$
F(s ; r, m):=\sum_{\substack{n \geqslant 1 \\ n \equiv c(\bmod m)}} \frac{\tau_{z}(r n)}{n^{s}} \quad(\sigma>1) .
$$

Nous pouvons écrire grâce aux relations d'orthogonalité des caractères

$$
F(s ; r, m)=\frac{1}{\varphi(m)} \sum_{\chi(\bmod m)} \bar{\chi}(c) F(s ; r, \chi)
$$

avec

$$
\begin{aligned}
F(s ; r, \chi) & :=\sum_{n \geqslant 1} \frac{\chi(n) \tau_{z}(r n)}{n^{s}}=\prod_{p \nmid r}\left(1-\frac{\chi(p)}{p^{s}}\right)^{-z} \prod_{p^{\nu} \| r} \sum_{j \geqslant 0} \frac{\tau_{z}\left(p^{\nu+j}\right) \chi\left(p^{j}\right)}{p^{j s}} \\
& =L(s, \chi)^{z} \prod_{\substack{p^{\nu} \| r \\
p \nmid m}}\left(1-\frac{\chi(p)}{p^{s}}\right)^{z} \sum_{j \geqslant 0} \frac{\tau_{z}\left(p^{\nu+j}\right) \chi\left(p^{j}\right)}{p^{j s}} \prod_{\substack{p^{\nu} \| r \\
p \mid m}} \tau_{z}\left(p^{\nu}\right) .
\end{aligned}
$$


On a en particulier, en notant $\chi_{0, m}$ le caractère principal de module $m$,

$$
F\left(s ; r, \chi_{0, m}\right)=\zeta(s)^{z} G(s, z ; r, m)
$$

avec

$$
G(s, z ; r, m):=\prod_{p \mid m r}\left(1-\frac{1}{p^{s}}\right)^{z} \prod_{\substack{p^{\nu} \| r \\ p \nmid m}} \sum_{j \geqslant 0} \frac{\tau_{z}\left(p^{\nu+j}\right)}{p^{j s}} \prod_{\substack{p^{\nu} \| r \\ p \mid m}} \tau_{z}\left(p^{\nu}\right) .
$$

En utilisant $(6 \cdot 20)$ et la sous-multiplicativité de $\tau_{\kappa}$, on montre aisément que l'on a pour $|s-1| \leqslant 1 / \log (2 \mathrm{rm})$

$$
|G(s, z ; r, m)| \ll \frac{\varphi(m)}{m} \tau_{\kappa}(r) \log (2 r m) .
$$

On conclut la démonstration en employant la méthode de Selberg-Delange (cf. [29], chap. II.5). La contribution des caractères non principaux est estimée grâce aux propriétés de prolongement analytique des fonctions $L(s, \chi)$ dans le demi-plan $\sigma<1$. Celle du caractère principal est évaluée en faisant appel à (13.15) et (13·16).

Nous sommes maintenant en mesure d'établir une estimation de $W_{\tau_{z}}(x, x ; \vartheta)$ valable uniformément pour $\vartheta \in \mathbb{R}$.

Lemme 13.6. Soient $A>0, \kappa \geqslant 1, B:=5\left(A+\kappa^{2}+\kappa+4\right)$. On a uniformément pour $x \geqslant 2, z \in \mathbb{C},|z| \leqslant \kappa, Q_{x}=x /(\log x)^{B}, \vartheta \in \mathbb{R}, q:=q\left(\vartheta ; Q_{x}\right)$,

$$
W_{\tau_{z}}(x, x ; \vartheta) \ll x(\log x)^{\Re e z-1}\left\{\frac{\tau_{\kappa+2}(q) \log q}{q}+\frac{1}{(\log x)^{A}}\right\} .
$$

Démonstration. Nous employons la méthode précédemment mise en œuvre dans les démonstrations de la Proposition 7.1 et du Lemme 11.3.

Lorsque $q$ satisfait aux hypothèses de l'énoncé, on a

$$
W_{\tau_{z}}(x, x ; a / q) \ll x /(\log x)^{A+\kappa+1} .
$$

Cela nous permet de disposer du cas $\vartheta \in \mathbb{Q}$.

Plaçons-nous maintenant dans le cas $\vartheta \in \mathbb{R} \backslash \mathbb{Q}$. Si $q=q\left(\vartheta ; Q_{x}\right)>(\log x)^{B}$, la majoration $(7 \cdot 4)$ fournit (13.17). Nous pouvons donc supposer dans la suite que $q \leqslant(\log x)^{B}$. Comme le membre de gauche de (13.17) est une fonction impaire de $\vartheta$, nous pouvons également, sans restreindre la généralité, supposer que, si $a \in \mathbb{Z}$ est tel que $(a, q)=1,|q \vartheta-a|=\|q \vartheta\| \leqslant 1 / Q_{x}$, alors $\vartheta_{q}:=\vartheta-a / q>0$.

Comme, d'après le Lemme 13.5 , on a $\tau_{z} \in S W^{+}$pour $|z| \leqslant \kappa$, nous pouvons évaluer $W_{\tau_{z}}(x, x ; \vartheta)$ à l'aide du Lemme 7.5 , les constantes impliquées ne dépendant que de $A, B$ et $\kappa$. Le nombre des indices $b$ dans la somme de (7.17) avec $f=\tau_{z}$ n'excédant pas $1+x\|q \vartheta\| \ll(\log x)^{B}$, il suffit, pour établir (13·17), de montrer que l'on a

$$
T_{b}^{(j)} \ll \frac{\tau_{\kappa+2}(q) \log q}{q}\left\{x_{b+1}-x_{b}\right\}(\log x)^{\Re e z-1}+\frac{x}{(\log x)^{B+A}}
$$

pour $0 \leqslant b \leqslant x\|q \vartheta\|$ et $1 \leqslant j \leqslant 3$. 
Commençons par majorer $T_{b}^{(1)}$. Il résulte immédiatement de (7·15) et (13·14) que

$$
\begin{aligned}
T_{b}^{(1)} & =\sum_{m \mid q} \frac{\gamma(m ; b / q)}{\varphi(m)} \sum_{\substack{x_{b} m / q<\ell \leqslant x_{b+1} m / q \\
(\ell, m)=1}} \tau_{z}\left(\frac{q \ell}{m}\right)+O\left(\frac{x}{(\log x)^{B+A}}\right) . \\
& \ll\left\{x_{b+1}-x_{b}\right\}(\log x)^{\Re e z-1} \frac{\log q}{q} \sum_{m \mid q} \tau(m) \tau_{\kappa}(q / m)+\frac{x}{(\log x)^{B+A}} \\
& \ll \frac{\tau_{\kappa+2}(q) \log q}{q}\left\{x_{b+1}-x_{b}\right\}(\log x)^{\Re e z-1}+\frac{x}{(\log x)^{B+A}},
\end{aligned}
$$

où nous avons utilisé la relation de convolution $\tau_{2} * \tau_{\kappa}=\tau_{\kappa+2}$. Cela garantit bien $(13 \cdot 18)$ pour $j=1$.

Nous estimons $T_{b}^{(2)}$ par sommation d'Abel à partir d'une évaluation classique de $\sum_{n \leqslant t} \tau_{z}(n)$. Il vient

$$
T_{b}^{(2)} \ll \vartheta_{q}\left(x_{b+1}-x_{b}\right)^{2}(\log x)^{\Re e z-1}+\vartheta_{q} x^{2} /(\log x)^{2 B+A} .
$$

Comme $\vartheta_{q}\left(x_{b+1}-x_{b}\right) \leqslant 1 / q$ et $\vartheta_{q} x \leqslant(\log x)^{B} / q$, cela implique (13.18) pour $j=2$.

Il reste à évaluer $T_{b}^{(3)}$. Compte tenu de (7.19), le Lemme 13.5 fournit

$$
T_{b}^{(3)} \ll \frac{\tau_{\kappa}(q) \log q}{q}\left\{x_{b+1}-x_{b}\right\}(\log x)^{\Re e z-1}+\frac{x}{(\log x)^{B+A}} .
$$

Cela achève la démonstration du Lemme 13.6.

Nous sommes maintenant en mesure d'établir la convergence de $V\left(\tau_{z} ; \vartheta\right)$ pour tout $z$ tel que $\Re e z<0$. Une sommation d'Abel fournit d'abord

$$
\sum_{n \leqslant x} \frac{\tau_{z}(n)}{n} B(n \vartheta)=\frac{W_{\tau_{z}}(x, x ; \vartheta)}{x}+\int_{1}^{x} W_{\tau_{z}}(t, t ; \vartheta) \frac{\mathrm{d} t}{t^{2}} .
$$

L'ensemble des $x$ satisfaisant à $q\left(\vartheta ; Q_{x}\right)=q_{m}$, c'est-à-dire $q_{m} \leqslant Q_{x}<q_{m+1}$, est un intervalle, que nous notons $\left[\xi_{m}, \xi_{m+1}\left[\right.\right.$. D'après $(6 \cdot 3)$, on a $\xi_{m} \asymp q_{m}\left(\log q_{m}\right)^{B}$. Ainsi que nous l'avons remarqué au paragraphe 11.2, on a $\log q_{m} \gg m$, donc également

$$
\log \xi_{m} \gg m \quad(m \geqslant 1) .
$$

Le Lemme 13.6 implique par conséquent pour $\Re e z<0$

$$
\begin{aligned}
\frac{W_{\tau_{z}}(x, x ; \vartheta)}{x} & \ll \frac{(\log x)^{\Re e z-1}}{\sqrt{q_{m}}}+\frac{1}{\left(\log \xi_{m}\right)^{A}} \ll \frac{1}{m^{A}} \quad\left(\xi_{m} \leqslant x \leqslant \xi_{m+1}\right) \\
\int_{\xi_{m}}^{\xi_{m+1}}\left|W_{\tau_{z}}(t, t ; \vartheta)\right| \frac{\mathrm{d} t}{t^{2}} & \ll \frac{\left(\log \xi_{m}\right)^{\Re e} z}{\sqrt{q_{m}}}+\frac{1}{\left(\log \xi_{m}\right)^{A-1}} \ll m^{1-A} .
\end{aligned}
$$


En particulier, la seconde majoration est, pour tout $A>2$, le terme général d'une série convergente. En reportant ces estimations dans (13·19), nous obtenons bien la convergence de $V\left(\tau_{z} ; \vartheta\right)$ sous l'hypothèse $\Re e z<0$.

\subsubsection{Convergence de $U\left(\tau_{z+1} ; \vartheta\right)$}

Nous établissons ici la convergence de la série $U\left(\tau_{z+1} ; \vartheta\right)$ lorsque $\Re e z \leqslant 0$.

Le cas $\vartheta \in \mathbb{Q}$ résulte immédiatement, par sommation d'Abel, de la formule (6.38) du Lemme 6.10 qui implique pour $a, q$ fixés et tout $A>0$

$$
Z_{\tau_{z}}(x, x ; a / q) \ll \frac{x}{(\log x)^{A}} .
$$

Nous omettons les détails, qui sont faciles.

Considérons maintenant le cas $\vartheta \in \mathbb{R} \backslash \mathbb{Q}$.

Une sommation d'Abel permet d'écrire

$$
\sum_{n \leqslant x} \tau_{z+1}(n) \frac{\sin (2 \pi n \vartheta)}{n}=\frac{Z_{\tau_{z+1}}(x, x ; \vartheta)}{x}+\int_{1}^{x} Z_{\tau_{z+1}}(t, t ; \vartheta) \frac{\mathrm{d} t}{t^{2}}
$$

Conservons la définition de la suite $\left\{\xi_{m}\right\}_{m=1}^{\infty}$ utilisée en (6·3). On déduit du Lemme 6.11 que l'on a, pour $x \in\left[\xi_{m}, \xi_{m+1}\right]$,

$$
\frac{Z_{\tau_{z+1}}(x, x ; \vartheta)}{x} \ll \frac{(\log x)^{\Re e} z}{\sqrt{q_{m}}} \frac{\log \left(1+\vartheta_{q}^{2} x^{2}\right)}{\left|\vartheta_{q}\right| x}+\frac{1}{(\log x)^{3}} \ll \frac{1}{\sqrt{q_{m}}}+\frac{1}{m^{3}}
$$

En majorant $(\log x)^{\Re e z}$ par 1 dans la première de ces estimations, nous obtenons

$$
\begin{aligned}
\int_{\xi_{m}}^{\xi_{m+1}}\left|Z_{\tau_{z+1}}(t, t ; \vartheta)\right| \frac{\mathrm{d} t}{t^{2}} & \ll \frac{1}{\sqrt{q_{m}}} \int_{0}^{\infty} \frac{\log \left(1+\vartheta_{q}^{2} t^{2}\right)\left|\vartheta_{q}\right| \mathrm{d} t}{\vartheta_{q}^{2} t^{2}}+\frac{1}{\left(\log \xi_{m}\right)^{2}} \\
& \ll \frac{1}{m^{2}} .
\end{aligned}
$$

La convergence de la série $U\left(\tau_{z+1} ; \vartheta\right)$ lorsque $\Re e z \leqslant 0$ résulte clairement de (13·22), $(13 \cdot 23)$ et $(13 \cdot 24)$. 


\section{Bibliographie}

[1] R.C. Baker \& G. Harman, Exponential sums formed with the Möbius function, J. London Math. Soc.(2) 43 (1991), 193-198.

[2] R. de la Bretèche, Sommes d'exponentielles et entiers sans grand facteur premier, Proc. London Math. Soc. (3) 77, (1998), 39-78.

[3] R. de la Bretèche, P-régularité de sommes d'exponentielles, Mathematika, 45 (1998), 145-175.

[4] R. de la Bretèche, Sommes sans grand facteur premier, Acta Arithmetica 88 no. 1 (1999), 1-14.

[5] N.G. de Bruijn, On the number of positive integers $\leqslant x$ and free of prime factors $>y$, Nederl. Akad. Wetensch. Proc. Ser. A 54 (1951), 50-60.

[6] S. Chowla, Some problems of diophantine approximation (I), Math. Z. 33 (1931), 544-563.

[7] H. Davenport, On some infinite series involving arithmetical functions, Quart. J. Math. Oxford 8 (1937), 8-13.

[8] H. Davenport, On some infinite series involving arithmetical functions (II), Quart. J. Math. Oxford, 8 (1937), 313-320.

[9] H. Davenport, Multiplicative number theory, deuxième édition, Graduate Texts in Mathematics 74 (Springer, Berlin, 1980).

[10] H. Davenport \& P. Erdös, On sequences of positive integers, Acta Arith. 2 (1937), 147-151.

[11] H. Davenport \& P. Erdős, On sequences of positive integers, J. Indian Math. Soc. 15 (1951), $19-24$.

[12] Y. Dupain, R.R. Hall \& G. Tenenbaum, Sur l'équirépartition modulo 1 de certaines fonctions de diviseurs, J. London Math. Soc. 26 (2) (1982), 397-411.

[13] E. Fouvry, Autour du théorème de Bombieri-Vinogradov, Acta Math. 152 (3-4), $219-244$.

[14] E. Fouvry \& G. Tenenbaum, Entiers sans grand facteur premier en progressions arithmétiques, Proc. London Math. Soc. (3) 63 (1991), 449-494.

[15] A. Hildebrand, The asymptotic behavior of the solutions of a class of differential-difference equations, Proc. London. Math. Soc. (2) 42 (1990), 11-31.

[16] M. Jutila, Lectures on a method in the theory of exponential sums, Tata Institute of Fundamental Research Lectures on Mathematics and Physics 80, Published for Tata Institute of Fundamental Research, Bombay by Springer-Verlag, Berlin-New York, 1987, viii+134 pp.

[17] T. Kano, On The size of $\sum_{n \leqslant x} d(n) \mathrm{e}(n \vartheta)$, Analytic number theory and diophantine problems, Proc. Conf., Stillwater/Okla. 1984, Prog. Math. 70, 283-290 (1987).

[18] Y. Katznelson, An introduction to harmonic analysis, Dover, New York, $2^{\mathrm{e}}$ édition, 1976 (1 ${ }^{\mathrm{e}}$ éd. 1968).

[19] A. Khintchine, Continued fractions, Noordhoff, Groningen, 1963.

[20] L. Kuipers \& H. Niederreiter, Uniform distribution of sequences, Wiley, New-York, 1976.

[21] E. Kummer, Bietrag zur Theorie der Function $\Gamma(x)=\int_{0}^{\infty} \mathrm{e}^{-v} v^{x-1} d x$, J. für Math., 35 (1847) $1-4$

[22] H. L. Montgomery \& R. C. Vaughan, Exponential sums with multiplicative coefficients, Invent. Math. 43 (1977), 69-82.

[23] W. Rudin, Analyse réelle et complexe, $3^{\mathrm{e}}$ édition, Dunod, 1998.

[24] S. L. Segal, On an identity between infinite series of arithmetic functions, Acta Arith. 28 (1976), $345-348$.

[25] H. Smida, Sur les puissance de convolution de la fonction de Dickman. Acta Arith.59 (1991), $124-143$.

[26] H. Smida, Valeur moyenne des fonctions de Piltz sur les entiers sans grand facteur premier, Acta Arith. 63, 1 (1993), 21-50.

[27] G. Tenenbaum, Facteur premier de sommes d'entiers, Proc. Amer. Math. Soc. 106 (1989), $287-296$.

[28] G. Tenenbaum, Sur un problème d'Erdős et Alladi, in : Séminaire de Théorie des Nombres (C. Goldstein, éd.), Paris 1988-89, Birkhäuser, Progress in Math. 91, 221-239.

[29] G. Tenenbaum, Introduction à la théorie analytique et probabiliste des nombres, $2^{\mathrm{e}}$ édition, Cours Spécialisés, n 1 , Société Mathématique de France, 1995.

[30] G. Tenenbaum, Uniform distribution on divisors and Behrend sequences, L'Ens. Math. 42 (2) (1996), 153-197.

[31] G. Tenenbaum, en collaboration avec Jie Wu, Exercices corrigés de théorie analytique et probabiliste des nombres, Cours spécialisés, n² , Société Mathématique de France, 1996. 
[32] E.T. Whittaker \& G.N. Watson, A course of modern analysis ( $4^{\mathrm{e}}$ éd.), Cambridge University Press, 1927 (réimpression 1986).

[33] J. R. Wilton, An approximate functional equation with applications to a problem of Diophantine approximation, J. reine angew. Math. 19 (1933), 219-237.

[34] J.-C. Yoccoz, Petits diviseurs en dimension 1, Astérisque 231 (1995).

[35] A. Zygmund, Trigonometric series, vol. 1 \& 2, 2e édition, Cambridge University Press, 1988.

Régis de la Bretèche

École Normale Supérieure

Département de Mathématiques et Applications

45 , rue d'Ulm

75230 Paris cedex 05

France
Gérald Tenenbaum

Institut Élie Cartan

Université de Nancy 1

BP 239

54506 Vandœuvre Cedex

France 